Akbar Zada, Hira Waheed, Jehad Alzabut*, and Xiaoming Wang

\title{
Existence and stability of impulsive coupled system of fractional integrodifferential equations
}

https://doi.org/10.1515/dema-2019-0035

Received April 8, 2019; accepted August 19, 2019

\begin{abstract}
In this manuscript, we deal with a class and coupled system of implicit fractional differential equations, having some initial and impulsive conditions. Existence and uniqueness results are obtained by means of Banach's contraction mapping principle and Krasnoselskii's fixed point theorem. Hyers-Ulam stability is investigated by using classical technique of nonlinear functional analysis. Finally, we provide illustrative examples to support our obtained results.
\end{abstract}

Keywords: Caputo fractional derivative; impulsive condition; existence and uniqueness theory; Hyers-Ulam stability

MSC: 26A33; 34A08; 35B40

\section{Introduction}

Fractional calculus is a generalization of ordinary differentiation and integration to arbitrary non-integer order, but with this definition, many interesting questions will arise; for example, if the first derivative of a function gives you the slope of the function, what is the geometrical meaning of half derivative? In half order, which operator must be used twice to obtain the first derivative? The early history of these questions goes back to the birth of fractional calculus in 1695 when Gottfried Wilhelm Leibniz suggested the possibility of fractional derivatives for the first time [1].

Fractional differential equations (FDEs) have recently gained much importance and attention. It is the extension of classical calculus. FDEs as well as fractional integrodifferential equations appear naturally as generalizations to existing models with integer derivatives and they also present new models for many applications in physics, control theory, chemistry, biology, electrical circuits, mechanics, signal and image processing, heat conduction, computer analysis and economics etc., reader is referred to [2-7]. For example, in the last three fields, some important considerations such as modeling, curve fitting, filtering, pattern recognition, edge detection, identification, stability, controllability, observability and robustness are now linked to long-range dependence phenomena. Similar progress has been made in other fields listed here.

Recently, the study of existence and uniqueness of solutions (EUSs) to initial and boundary value problems for FDEs has attracted considerable attention, we refer to [8-17] and the references therein. A large number of methods are used in investigating the EUSs for FDEs, such as comparison methods, fixed point

\footnotetext{
Akbar Zada, Hira Waheed: Department of Mathematics, University of Peshawar, Peshawar 25000, Pakistan; E-mail: zadababo@yahoo.com, hirams2017@gmail.com

*Corresponding Author: Jehad Alzabut: Department of Mathematics and General Sciences, Prince Sultan University, 11586 Riyadh, Saudi Arabia; E-mail: jalzabut@psu.edu.sa

Xiaoming Wang: School of Mathematics \& Computer Science, Shangrao Normal University, Shangrao 334001, China; E-mail: wxmsuda03@163.com
} 
method and coincidence degree method etc $[18,19]$. EUSs for FDEs in finite dimensional as well as infinite dimensional spaces were studied by several authors [20-25].

The study of coupled system (CS) of FDEs has also attracted some attention. Because mathematical models of various phenomena in the field of biology, physics and psychology etc. are in the form of CS of differential equations (DEs). For the study of CS of FDEs, we refer the reader to [26-31].

Another important class of DEs is known as impulsive differential equations (IDEs). This class plays the role of an effective mathematical tools for those evolution processes that are subject to abrupt changes in their states. There are many physical phenomena that exhibit impulsive behavior such as the maintenance of a species through periodic stocking or harvesting, mechanical systems subject to impacts, the thrust impulse maneuver of a spacecraft and the function of heart, we recommended [32-41] for more details on the theory of IDEs. It is well known that in the evolution processes the impulsive phenomena can be found in many situations. For example, change of the valve shutter speed in its transition from open to closed state [42], operation of a damper subjected to the percussive effects [43], disturbances in cellular neural networks [44, 45], relaxational oscillations of the electro mechanical systems [46], percussive systems with vibrations [47], using the radial acceleration, control of the satellite orbit [48], dynamic of system with automatic regulation [48], fluctuations of pendulum systems in the case of external impulsive effects [49], price fluctuations in commodity markets [50] and so on.

Furthermore, stability analysis plays an important role in the systems of DEs, numerical analysis, economics, optimization theory etc. In the literature, we can find different types of stability such as Mittag-Lefler stability, Lyapunov stability, Exponential stability and Hyers-Ulam (HU) stability [51-53]. Recently, HU stability is interesting for the stability analysis of FDEs. Many manuscripts are devoted to HU stability see [54-62].

In this manuscript, we study four different types of Ulam stability for implicit FDEs with impulses and initial conditions, which are HU stability, generalized HU stability, HU-Rassias stability and generalized HURassias stability.

In [63], Wang et al. developed sufficient conditions for the following problem:

$$
\left\{\begin{array}{l}
{ }^{c} D^{\mathfrak{q}} u(t)=\mathrm{f}(t, u(t)), t \in[0, \mathrm{~T}], \mathfrak{D}:=\left\{t_{1}, t_{2}, \ldots, t_{m}\right\}, \\
u(0)=u_{0}, \\
\Delta u\left(t_{i}\right)=I_{i}\left(u\left(t_{i}\right)\right),
\end{array}\right.
$$

where $0<\mathfrak{q}<1, \mathrm{f}:[0, \mathrm{~T}] \times \mathbb{R} \rightarrow \mathbb{R}$ is a continuous function and $I_{i}: \mathbb{R} \rightarrow \mathbb{R}$ are nonlinear functions describing the jump size $I_{i}\left(u\left(t_{i}\right)\right)=u\left(t_{i}^{+}\right)-u\left(t_{i}^{-}\right)$at $t_{i}, 0=t_{0}<t_{1}<\cdots<t_{m}<t_{m+1}=\mathrm{T}$.

Tian et al. [64] investigated the existence of positive solutions to the following impulsive FDE:

$$
\left\{\begin{array}{l}
{ }^{c} D^{\mathfrak{r}} u(t)=\Phi(t, u(t)), 0 \leq t \leq 1, t \neq t_{k}, k=1,2, \ldots, p, \\
\Delta u\left(t_{k}\right)=I_{k}\left(u\left(t_{k}\right)\right), \Delta u^{\prime}\left(t_{k}\right)=\bar{I}_{k}\left(u\left(t_{k}\right)\right), k=1,2, \ldots, p, \\
u(0)=\mathrm{g}(u), u(1)=\mathrm{h}(u),
\end{array}\right.
$$

where $1<\mathfrak{r} \leq 2, \Phi:[0,1] \times \mathbb{R} \rightarrow \mathbb{R}$ and $I_{k}, \bar{I}_{k}: \mathbb{R} \rightarrow \mathbb{R}$ are continuous functions and $\Delta\left(u\left(t_{k}\right)\right)=u\left(t_{k}^{+}\right)-u\left(t_{k}^{-}\right)$, $\Delta\left(u^{\prime}\left(t_{k}^{+}\right)\right)=u^{\prime}\left(t_{k}^{+}\right)-u^{\prime}\left(t_{k}^{-}\right)$with $u\left(t_{k}^{+}\right), u^{\prime}\left(t_{k}^{+}\right), u\left(t_{k}^{-}\right), u^{\prime}\left(t_{k}^{-}\right)$, are the respective right and left limits of $u\left(t_{k}\right)$ at $t=$ $t_{k}$.

Zhang et al. [65] extended the work to CS of $2 \mathrm{~m}$-point boundary value problem for impulsive FDEs at resonance as:

$$
\left\{\begin{array}{l}
D_{0^{+}}^{\mathfrak{r}} u(t)=\mathrm{F}\left(t, v(t), D^{\mathfrak{p}} v(t)\right), D_{0^{+}}^{\mathfrak{s}} v(t)=\mathrm{G}\left(t, u(t), D^{\mathfrak{q}} u(t)\right), 0<t<1, \\
\Delta u\left(t_{i}\right)=A_{i}\left(v\left(t_{i}\right), D^{\mathfrak{p}} v\left(t_{i}\right)\right), \Delta D_{0^{+}}^{\mathfrak{q}} u\left(t_{i}\right)=B_{i}\left(v\left(t_{i}\right), D^{\mathfrak{p}} v\left(t_{i}\right)\right), i=1,2, \ldots, k, \\
\Delta v\left(t_{i}\right)=C_{i}\left(u\left(t_{i}\right), D^{\mathfrak{q}} u\left(t_{i}\right)\right), \Delta D_{0^{+}}^{\mathfrak{p}} v\left(t_{i}\right)=D_{i}\left(u\left(t_{i}\right), D^{\mathfrak{q}} u\left(t_{i}\right)\right), i=1,2, \ldots, k, \\
D_{0^{+}}^{\mathfrak{r}-1}=\sum_{i=1}^{m} a_{i} D^{\mathfrak{r}-1} u\left(\xi_{i}\right), u(1)=\sum_{i=1}^{m} b_{i} \eta_{i}^{2-\mathfrak{r}} u\left(\eta_{i}\right), \\
D_{0^{+}}^{\mathfrak{s}-1}=\sum_{i=1}^{m} c_{i} D^{\mathfrak{s}-1} v\left(\xi_{i}\right), v(1)=\sum_{i=1}^{m} d_{i} \theta_{i}^{2-\mathfrak{s}} v\left(\theta_{i}\right),
\end{array}\right.
$$


where $1<\mathfrak{r}, \mathfrak{s}<2, \mathfrak{r}-\mathfrak{q} \geq 1, \mathfrak{s}-\mathfrak{p} \geq 1$ and $\mathrm{F}, \mathrm{G}:(0,1) \times \mathbb{R}^{2} \rightarrow \mathbb{R}$ are continuous functions.

Shah et al. [66] investigated the EUSs of:

$$
\left\{\begin{array}{l}
{ }^{c} D^{\alpha} x(t)=\mathrm{f}(t, x(t), y(t)), 0 \leq t \leq 1, t \neq t_{j}, j=1,2, \ldots, m, \\
{ }^{c} D^{\beta} y(t)=\mathrm{g}(t, x(t), y(t)), 0 \leq t \leq 1, t \neq t_{i}, i=1,2, \ldots, n, \\
x(0)=\mathrm{h}(x), x(1)=\mathrm{g}(x) \text { and } y(0)=\mathrm{k}(y), y(1)=\mathrm{f}(y), \\
\Delta x\left(t_{j}\right)=I_{j}\left(x\left(t_{j}\right)\right), \Delta x^{\prime}\left(t_{j}\right)=\bar{I}_{j}\left(x\left(t_{j}\right)\right), j=1,2, \ldots, m, \\
\Delta y\left(t_{i}\right)=I_{i}\left(y\left(t_{i}\right)\right), \Delta y^{\prime}\left(t_{i}\right)=\bar{I}_{i}\left(y\left(t_{i}\right)\right), i=1,2, \ldots, n,
\end{array}\right.
$$

where $\alpha, \beta \in(1,2], \mathrm{f}, \mathrm{g}:[0,1] \times \mathbb{R}^{2} \rightarrow \mathbb{R}$ are continuous functions.

Benchohra and Lazreg [67] investigated the implicit FDEs:

$$
\left\{\begin{array}{l}
{ }^{c} D^{\alpha} u(t)=\Phi\left(t, u(t),{ }^{c} D^{\alpha} u(t)\right), 0 \leq t \leq 1,0<\alpha \leq 1, \\
u(0)=u_{0},
\end{array}\right.
$$

where ${ }^{c} D^{\alpha}$ is the Caputo derivative of fractional order $\alpha$ and $\Phi:(0,1] \times \mathbb{R}^{2} \rightarrow \mathbb{R}$ is continuous function, $u_{0} \in \mathbb{R}$.

Zada et al. [68] studied the EUSs to the implicit FDE of the form:

$$
\left\{\begin{array}{l}
{ }^{c} D_{0, t}^{\beta} y(t)=\mathcal{G}\left(t, y(t),{ }^{c} D_{0, t}^{\beta} y(t)\right), t \in\left(t_{k}, s_{k}\right], k=0,1, \ldots, m, \beta \in(0,1], \\
y(t)=I_{s-1, t_{k}}^{\beta}\left(\xi_{k}(t, y(t))\right), t \in\left(s_{k-1}, t_{k}\right], k=1,2, \ldots, m, \\
y(0)=I_{0, T}^{\beta} \eta(t, y(t)),
\end{array}\right.
$$

where ${ }^{c} D_{0, t}^{\beta}$ is the Caputo fractional derivative of order $\beta$ with lower limit 0 and $\mathcal{G}:(0,1] \times \mathbb{R}^{2} \rightarrow \mathbb{R}$ is a continuous function.

In this manuscript, we study the existence, uniqueness and HU stability results of the implicit FDE with impulsive condition as:

$$
\left\{\begin{array}{l}
{ }^{c} D^{\mathfrak{r}} x(t)=\mathcal{F}\left(t, x(t),{ }^{c} D^{\mathfrak{r}} x(t)\right)+\int_{0}^{t} \frac{(t-\xi)}{\Gamma(\delta)} \mathcal{G}\left(\xi, x(\xi),{ }^{c} D^{r} x(\xi)\right) d \xi, t \in \mathcal{J}, t \neq t_{i} \text { for } i=1,2, \ldots, m, \\
x(0)=\mathrm{h}(x), \\
\Delta x\left(t_{i}\right)=I_{i}\left(x\left(t_{i}\right)\right), \quad i=1,2, \ldots, m,
\end{array}\right.
$$

where $0<\mathfrak{r}<1, \mathcal{J}=[0, \mathrm{~T}]$ with $\mathrm{T}>0, \sigma, \delta>0$. The functions $\mathcal{F}, \mathcal{G}: \mathcal{J} \times \mathbb{R}^{2} \rightarrow \mathbb{R}$ and $\mathrm{h}: \mathcal{X} \rightarrow \mathbb{R}$ are continuous functions. Also we investigate the aforementioned analysis for the proposed implicit CS:

$$
\left\{\begin{array}{l}
{ }^{c} D^{\mathfrak{r}} x(t)=\mathcal{F}\left(t, y(t),{ }^{c} D^{\mathfrak{r}} x(t)\right)+\int_{0}^{t} \frac{(t-\xi)}{\Gamma(\delta)} \mathcal{G}\left(\xi, y(\xi),{ }^{c} D^{\mathfrak{r}} x(\xi)\right) d \xi, t \in \mathcal{J}, t \neq t_{i} \text { for } i=1,2, \ldots, m, \\
{ }^{c} D^{\mathfrak{s}} y(t)=\mathcal{F}^{\prime}\left(t, x(t),{ }^{c} D^{\mathfrak{s}} y(t)\right)+\int_{0}^{t} \frac{(t-\xi)^{\sigma-1}}{\Gamma(\delta)} \mathcal{G}^{\prime}\left(\xi, x(\xi),{ }^{c} D^{\mathfrak{s}} y(\xi)\right) d \xi, t \in \mathcal{J}, t \neq t_{j} \text { for } j=1,2, \ldots, n, \\
x(0)=\mathrm{h}(x), y(0)=\mathrm{g}(y), \\
\Delta x\left(t_{i}\right)=I_{i}\left(x\left(t_{i}\right)\right), \quad i=1,2, \ldots, m, \\
\Delta y\left(t_{j}\right)=I_{j}\left(y\left(t_{j}\right)\right), \quad j=1,2, \ldots, n,
\end{array}\right.
$$

where $0<\mathfrak{r}, \mathfrak{s}<1, \sigma, \delta>0$ and $\mathcal{J}=[0, \mathrm{~T}]$ with $\mathrm{T}>0$. The functions $\mathcal{F}, \mathcal{G}, \mathcal{F}^{\prime}, \mathcal{G}^{\prime}: \mathcal{J} \times \mathbb{R}^{2} \rightarrow \mathbb{R}$ and $\mathrm{h}: X \rightarrow \mathbb{R}, \mathrm{g}: \mathrm{y} \rightarrow \mathbb{R}$ are continuous.

Notation: Let $\mathcal{J}=[0, \mathrm{~T}]$. We denote $P C(\mathcal{J}, \mathbb{R})$ by $\mathcal{M}$ i.e the space of all piecewise continuous functions. The interval $\mathcal{J}=\mathcal{J}_{0} \cup \mathcal{J}_{1} \cup \mathcal{J}_{2} \cup \cdots \cup \mathcal{J}_{i}$, where $\mathcal{J}_{0}=\left[t_{0}, t_{1}\right], \mathcal{J}_{1}=\left(t_{1}, t_{2}\right], \mathcal{J}_{2}=\left(t_{2}, t_{3}\right], \ldots, \mathcal{J}_{i}=\left(t_{i}, t_{i+1}\right], i=$ 
$1,2, \ldots, m$ and $\mathcal{J}^{\prime}=\mathcal{J}-\left\{t_{1}, t_{2}, t_{3}, \ldots, t_{i}\right\}$.

We define $\mathcal{M}=\left\{x: \mathcal{J} \rightarrow \mathbb{R}: x \in C\left(\mathcal{J}_{i}, \mathbb{R}\right)\right.$ and $x\left(t_{i}^{+}\right), x\left(t_{i}^{-}\right)$exist so that $\Delta x\left(t_{i}\right)=x\left(t_{i}^{+}\right)-x\left(t_{i}^{-}\right)$for $\left.i=1,2, \ldots, m\right\}$.

The rest of this paper is arranged as follows: In section 2, we present some basic notions needed to prove our main results. In section 3, we setup some adequate conditions for the EUSs, by applying some standard fixed point principles to the proposed system (1.1) and (1.2), respectively. In section 4, we setup applicable results under which the solution of the considered problems (1.1) and (1.2), respectively, fulfills the conditions of different kinds of Ulam stability. The establish results are illustrated by examples in section 5 .

\section{Supplementary results}

The following definitions and lemmas are adopted from [18].

Definition 2.1. The integral of a function $u \in L^{1}(\mathcal{J}, \mathbb{R})$ of order $\mathfrak{r} \in(0, \infty)$ is defined by

$$
I^{\mathfrak{r}} u(t)=\frac{1}{\Gamma(\mathfrak{r})} \int_{0}^{t}(t-\xi)^{r-1} u(\xi) d \xi,
$$

where

$$
\Gamma(\mathfrak{r})=\int_{0}^{\infty} t^{\mathfrak{r}-1} e^{-t} d t ; \mathfrak{r}>0 .
$$

Definition 2.2. The Caputo arbitrary order $\mathfrak{r}$ derivative of function $u \in C^{(n)}((0, \infty), \mathbb{R})$ is defined by

$$
{ }^{c} D^{\mathfrak{r}} u(t)=\frac{1}{\Gamma(\rho-\mathfrak{r})} \int_{0}^{t}(t-\xi)^{\rho-r-1} u^{(n)}(\xi) d \xi,
$$

where $\rho=[\mathfrak{r}]+1$ in which $[\mathfrak{r}]$ represents the integer part of $\mathfrak{r}$.

Lemma 2.3. For $\mathfrak{r}>0$, the following result hold:

$$
I^{\mathfrak{r}}\left[{ }^{c} D^{\mathfrak{r}} u(t)\right]=u(t)-\sum_{\rho=0}^{n-1} \frac{u^{(\rho)}(0)}{\rho !} t^{\rho},
$$

where $n=[r]+1$.

Lemma 2.4. For $\mathfrak{r}>0$, the solution of FDE

$$
{ }^{c} D^{\mathfrak{r}} u(t)=\beta(t)
$$

is given by

$$
u(t)=I^{\mathfrak{r}} \beta(t)+\sum_{\rho=0}^{n-1} \frac{u^{(\rho)}(0)}{\rho !} t^{\rho},
$$

where $n=[\mathfrak{r}]+1$.

Theorem 2.5. [69] Let $\mathcal{M}$ be a Banach space, $\mathcal{T}: \mathcal{M} \rightarrow \mathcal{M}$ be a completely continuous operator, and the set $\Omega=\{x \in \aleph: x=\aleph \mathcal{T} x, 0<\aleph<1\}$ is bounded, then $\mathcal{T}$ has at least one fixed point in $\mathcal{M}$.

Theorem 2.6. [69] Let $\mathcal{B}$ be a non-empty closed subset of a Banach space $\mathcal{M}$. Then any contraction mapping $\mathcal{T}$ of $\mathcal{B}$ into itself has a unique fixed point. 
Theorem 2.7. [70] Let $\mathcal{H}$ be a convex closed and non-empty subset of Banach space $X \times y$ and $\mathbb{F}$, $\mathbb{G}$ be the operators so that

(i) $\mathbb{F} x+\mathbb{G} y \in \mathcal{H}$ whenever $x, y \in \mathcal{H}$.

(ii) $\mathbb{F}$ is compact and continuous and $\mathbb{G}$ is contraction mapping.

Then $\exists z \in \mathcal{H}$ so that $z=\mathbb{F} z+\mathbb{G} z$, where $z=(x, y) \in \mathcal{X} \times y$.

\section{Existence and uniqueness}

Here we present our result about the existence of at least one solution to considered problem (1.1).

Theorem 3.1. Let $0<\mathfrak{r} \leq 1$ and $\alpha \in \mathcal{M}$ be a continuous function, then a function $x \in \mathcal{M}$ is solution to problem

$$
\left\{\begin{array}{l}
{ }^{c} D^{\mathrm{r}} x(t)=\alpha(t), t \in \mathcal{J}, t \neq t_{i}, \text { for } i=1,2, \ldots, m, \\
x(0)=\mathrm{h}(x), \\
\Delta x\left(t_{i}\right)=I_{i}\left(x\left(t_{i}\right)\right), i=1,2, \ldots, m,
\end{array}\right.
$$

where

$$
\alpha(t)=\mathcal{F}\left(t, x(t),{ }^{c} D^{\mathfrak{r}} x(t)\right)+\int_{0}^{t} \frac{(t-\xi)^{\sigma-1}}{\Gamma(\delta)} \mathcal{G}\left(\xi, x(\xi),{ }^{c} D^{\mathfrak{r}} x(\xi)\right) d \xi,
$$

if and only if $x$ satisfies

$$
x(t)=\left\{\begin{array}{l}
\frac{1}{\Gamma(\mathfrak{r})} \int_{0}^{t}(t-\xi)^{\mathfrak{r}-1} \alpha(\xi) d \xi+\mathrm{h}(x), t \in \mathcal{J}_{0}, \\
\frac{1}{\Gamma(\mathfrak{r})} \int_{t_{i}}^{t}(t-\xi)^{\mathfrak{r}-1} \alpha(\xi) d \xi+\sum_{i=1}^{m}\left[\frac{1}{\Gamma(\mathfrak{r})} \int_{t_{i-1}}^{t_{i}}\left(t_{i}-\xi\right)^{\mathfrak{r}-1} \alpha(\xi) d \xi+I_{i}\left(x\left(t_{i}\right)\right)\right]+\mathrm{h}(x), t \in \mathcal{J}_{i}, i=1,2, \ldots, m .
\end{array}\right.
$$

Proof. Let $x$ be the solution of problem (3.1), then using Lemma 2.4, for each $t \in \mathcal{J}_{0}$, we have

$$
x(t)=I^{\mathfrak{r}} \alpha(t)+x(0)=\frac{1}{\Gamma(\mathfrak{r})} \int_{0}^{t}(t-\xi)^{\mathfrak{r}-1} \alpha(\xi) d \xi+x(0) .
$$

Using the initial condition $x(0)=\mathrm{h}(x)$, we get from (3.3)

$$
x(t)=\frac{1}{\Gamma(\mathfrak{r})} \int_{0}^{t}(t-\xi)^{\mathfrak{r}-1} \alpha(\xi) d \xi+\mathrm{h}(x), t \in \mathcal{J}_{0} .
$$

Similarly, for $t \in \mathcal{J}_{1}$,

$$
x(t)=\frac{1}{\Gamma(\mathfrak{r})} \int_{t_{1}}^{t}(t-\xi)^{\mathfrak{r}-1} \alpha(\xi) d \xi+x\left(t_{1}\right)
$$

we have

$$
x\left(t_{1}^{-}\right)=\frac{1}{\Gamma(\mathfrak{r})} \int_{0}^{t}(t-\xi)^{\mathfrak{r}-1} \alpha(\xi) d \xi+\mathrm{h}(x), x\left(t_{1}^{+}\right)=x\left(t_{1}\right) .
$$

From

$$
\Delta x\left(t_{1}\right)=x\left(t_{1}^{+}\right)-x\left(t_{1}^{-}\right)=I_{1}\left(x\left(t_{1}\right)\right),
$$


we get

$$
x\left(t_{1}\right)=\frac{1}{\Gamma(\mathfrak{r})} \int_{0}^{t}(t-\xi)^{\mathfrak{r}-1} \alpha(\xi) d \xi+\mathrm{h}(x)+I_{1}\left(x\left(t_{1}\right)\right), t \in \mathcal{J}_{1} .
$$

Putting for $x\left(t_{1}\right),(3.5)$ implies

$$
x(t)=\frac{1}{\Gamma(\mathfrak{r})} \int_{t_{1}}^{t}(t-\xi)^{\mathfrak{r}-1} \alpha(\xi) d \xi+\frac{1}{\Gamma(\mathfrak{r})} \int_{0}^{t}(t-\xi)^{\mathfrak{r}-1} \alpha(\xi) d \xi+\mathrm{h}(x)+I_{1}\left(x\left(t_{1}\right)\right), t \in \mathcal{J}_{1} .
$$

Generalizing in this way, for $t \in \mathcal{J}_{i}$, we have

$$
x(t)=\frac{1}{\Gamma(\mathfrak{r})} \int_{t_{i}}^{t}(t-\xi)^{\mathfrak{r}-1} \alpha(\xi) d \xi+\sum_{i=1}^{m}\left[\frac{1}{\Gamma(\mathfrak{r})} \int_{t_{i-1}}^{t_{i}}\left(t_{i}-\xi\right)^{\mathfrak{r}-1} \alpha(\xi) d \xi+I_{i}\left(x\left(t_{i}\right)\right)\right]+\mathrm{h}(x), i=1,2, \ldots, m .
$$

Using (3.4) and (3.6), we obtain (3.2).

Conversely, let $x$ be the solution of integral equation (3.2), then the $r$ th order derivative of (3.2) will lead us to the first equation in (3.1). Further, it is easy to obtain the initial and impulsive conditions of (3.1).

Corollary 3.2. In light of Theorem 3.1, problem (1.1) has the following solution:

$$
x(t)=\left\{\begin{array}{l}
\frac{1}{\Gamma(\mathfrak{r})} \int_{0}^{t}(t-\xi)^{\mathfrak{r}-1} \alpha(\xi) d \xi+\mathrm{h}(x), t \in \mathcal{J}_{0}, \\
\frac{1}{\Gamma(\mathfrak{r})} \int_{t_{i}}^{t}(t-\xi)^{\mathfrak{r}-1} \alpha(\xi) d \xi+\sum_{i=1}^{m}\left[\frac{1}{\Gamma(\mathfrak{r})} \int_{t_{i-1}}^{t_{i}}\left(t_{i}-\xi\right)^{\mathfrak{r}-1} \alpha(\xi) d \xi+I_{i}\left(x\left(t_{i}\right)\right)\right]+\mathrm{h}(x), t \in \mathcal{J}_{i}, i=1,2, \ldots, m,
\end{array}\right.
$$

where

$$
\alpha(t)=\mathcal{F}\left(t, x(t),{ }^{c} D^{\mathfrak{r}} x(t)\right)+\int_{0}^{t} \frac{(t-\xi)^{\sigma-1}}{\Gamma(\delta)} \mathcal{G}\left(\xi, x(\xi),{ }^{c} D^{\mathfrak{r}} x(\xi)\right) d \xi .
$$

For simplicity, we use the following notation:

$$
\begin{aligned}
\tilde{x}(t) & =\mathcal{F}\left(t, x(t),{ }^{c} D^{\mathfrak{r}} x(t)\right)+\int_{0}^{t} \frac{(t-\xi)^{\sigma-1}}{\Gamma(\delta)} \mathcal{G}\left(\xi, x(\xi),{ }^{c} D^{\mathfrak{r}} x(\xi)\right) d \xi \\
& =\mathcal{F}(t, x(t), \tilde{x}(t))+\int_{0}^{t} \frac{(t-\xi)^{\sigma-1}}{\Gamma(\delta)} \mathcal{G}(\xi, x(\xi), \tilde{x}(\xi)) d \xi
\end{aligned}
$$

Let $\mathcal{M}=P C(\mathcal{g}, \mathbb{R})$ be a Banach space endowed with norm

$$
\|x\|_{\mathcal{M}}=\max \{|x(t)|: t \in \mathcal{J}\} .
$$

If $x$ is a solution of the problem (1.1), then

$$
x(t)=\frac{1}{\Gamma(\mathfrak{r})} \int_{t_{i}}^{t}(t-\xi)^{\mathfrak{r}-1} \alpha(\xi) d \xi+\sum_{i=1}^{m}\left[\frac{1}{\Gamma(\mathfrak{r})} \int_{t_{i-1}}^{t_{i}}(t-\xi)^{\mathfrak{r}-1} \alpha(\xi) d \xi+I_{i}\left(x\left(t_{i}\right)\right)\right]+\mathrm{h}(x), \forall t \in \mathcal{J}_{i}, i=1,2, \ldots, m .
$$


To transform problem (1.1) into a fixed point problem, we define an operator $\mathcal{T}: \mathcal{M} \rightarrow \mathcal{M}$ by

$$
(\mathcal{T} x)(t)=\left\{\begin{array}{l}
\frac{1}{\Gamma(\mathfrak{r})} \int_{0}^{t}(t-\xi)^{\mathfrak{r}-1} \tilde{\chi}(\xi) d \xi+\mathrm{h}(x), t \in \mathcal{J}_{0}, \\
\frac{1}{\Gamma(\mathfrak{r})} \int_{t_{i}}^{t}(t-\xi)^{\mathfrak{r}-1} \tilde{\chi}(\xi) d \xi+\sum_{i=1}^{m}\left[\frac{1}{\Gamma(\mathfrak{r})} \int_{t_{i-1}}^{t_{i}}\left(t_{i}-\xi\right)^{\mathfrak{r}-1} \tilde{\chi}(\xi) d \xi+I_{i}\left(x\left(t_{i}\right)\right)\right]+\mathrm{h}(x), t \in \mathcal{J}_{i}, \quad i=1,2, \ldots, m,
\end{array}\right.
$$

where

$$
\tilde{x}(t)=\mathcal{F}(t, x(t), \tilde{x}(t))+\int_{0}^{t} \frac{(t-\xi)^{\sigma-1}}{\Gamma(\delta)} \mathcal{G}(\xi, x(\xi), \tilde{x}(\xi)) d \xi .
$$

For our next results, we put the following hypotheses.

Assume that

- $\left[A_{1}\right]$ there exist constants $\mathrm{M}_{1}>0$ and $0<\mathrm{N}_{1}<1$ so that for each $t \in \mathcal{J}$ and for all $u, \bar{u} \in \mathcal{M}$ and $w, \bar{w} \in \mathbb{R}$, the following relation holds

$$
|\mathcal{F}(t, u, w)-\mathcal{F}(t, \bar{u}, \bar{w})| \leq \mathrm{M}_{1}|u-\bar{u}|+\mathrm{N}_{1}|w-\bar{w}| ;
$$

Similarly, there exist constants $\mathrm{M}_{2}>0$ and $0<\mathrm{N}_{2}<1$ so that for each $t \in \mathcal{J}$ and for all $u, \bar{u} \in \mathcal{M}$ and $w, \bar{w} \in \mathbb{R}$ the following relation holds

$$
|\mathcal{G}(t, u, w)-\mathcal{G}(t, \bar{u}, \bar{w})| \leq \mathrm{M}_{2}|u-\bar{u}|+\mathrm{N}_{2}|w-\bar{w}| ;
$$

- $\left[A_{2}\right]$ for any $u, \bar{u} \in \mathcal{M}, \exists$ a constant $A_{I_{i}}>0$ so that

$$
\left|I_{i}\left(u\left(t_{i}\right)\right)-I_{i}\left(\bar{u}\left(t_{i}\right)\right)\right| \leq A_{I_{i}}\left|u\left(t_{i}\right)-\bar{u}\left(t_{i}\right)\right| ;
$$

- $\left[A_{3}\right]$ for any $u, w \in \mathbb{R}, \exists$ a constant $A_{\mathrm{h}}>0$ so that

$$
|\mathrm{h}(u)-\mathrm{h}(w)| \leq A_{\mathrm{h}}|u-w| ;
$$

- $\left[A_{4}\right]$ there exist bounded functions $a_{1}, b_{1}, c_{1} \in \mathcal{M}$ so that

$$
|\mathcal{F}(t, u(t), w(t))| \leq a_{1}(t)+b_{1}(t)|u(t)|+c_{1}(t)|w(t)|
$$

with $a_{1}^{\star}=\sup _{t \in \mathcal{J}} a_{1}(t), b_{1}^{\star}=\sup _{t \in \mathcal{J}} b_{1}(t), c_{1}^{\star}=\sup _{t \in \mathcal{J}} c_{1}(t)<1$;

Similarly, there exist bounded functions $a_{2}, b_{2}, c_{2} \in \mathcal{M}$ so that

$$
|\mathcal{G}(t, u(t), w(t))| \leq a_{2}(t)+b_{2}(t)|u(t)|+c_{2}(t)|w(t)|
$$

with $a_{2}^{\star}=\sup _{t \in \mathcal{J}} a_{2}(t), b_{2}^{\star}=\sup _{t \in \mathcal{J}} b_{2}(t), c_{2}^{\star}=\sup _{t \in \mathcal{J}} c_{2}(t)<1$;

- $\left[A_{5}\right] \exists$ a constant $\lambda>0$ so that $|\mathrm{h}(u)| \leq \lambda$, for all $u \in \mathcal{M}$;

- $\left[A_{6}\right]$ for each $u \in \mathbb{R}$, the function $I_{i}: \mathbb{R} \rightarrow \mathbb{R} ; i=1,2, \ldots, m$ are assumed to be continuous and for constants $\mathcal{K}, \mathcal{L}>0$, the inequality $\left|I_{i}\left(u\left(t_{i}\right)\right)\right| \leq \mathcal{K}|u(t)|+\mathcal{L}$, holds.

The existence of solution for the problem (1.1) is based on Theorem 2.5.

Theorem 3.3. Problem (1.1) has at least one solution if the hypothesis $\left[A_{1}\right]-\left[A_{6}\right]$ are satisfied.

Proof. Let the operator $\mathcal{T}$ is defined in (3.7). We need to prove that (1.1) has at least one solution. Let the operator $\mathcal{T}$ be continuous. Consider a sequence $\left\{x_{n}\right\}$ so that $x_{n} \rightarrow x \in \mathcal{M}, t \in \mathcal{J}$, then

$$
\left|\left(\mathcal{T} x_{n}\right)(t)-(\mathcal{T} x)(t)\right| \leq \frac{1}{\Gamma(\mathfrak{r})} \int_{t_{i}}^{t}(t-\xi)^{\mathfrak{r}-1}\left|\tilde{x}_{n}(\xi)-\tilde{x}(\xi)\right| d \xi+\sum_{i=1}^{m} \frac{1}{\Gamma(\mathfrak{r})} \int_{t_{i-1}}^{t_{i}}\left(t_{i}-\xi\right)^{\mathfrak{r}-1}\left|\tilde{x}_{n}(\xi)-\tilde{x}(\xi)\right| d \xi
$$




$$
+\sum_{i=1}^{m}\left|I_{i}\left(x_{n}\left(t_{i}\right)\right)-I_{i}\left(x\left(t_{i}\right)\right)\right|+\left|\mathrm{h}\left(x_{n}\right)-\mathrm{h}(x)\right|, i=1,2, \ldots, m,
$$

where $\tilde{x}_{n}, \tilde{x} \in \mathcal{M}$ and are given by

$$
\tilde{x}_{n}(t)=\mathcal{F}\left(t, x_{n}(t), \tilde{x}_{n}(t)\right)+\int_{0}^{t} \frac{(t-\xi)^{\sigma-1}}{\Gamma(\delta)} \mathcal{G}\left(\xi, x_{n}(\xi), \tilde{x}_{n}(\xi)\right) d \xi
$$

and

$$
\tilde{x}(t)=\mathcal{F}(t, x(t), \tilde{x}(t))+\int_{0}^{t} \frac{(t-\xi)^{\sigma-1}}{\Gamma(\delta)} \mathcal{G}(\xi, x(\xi), \tilde{x}(\xi)) d \xi
$$

By utilizing $\left[A_{1}\right]$, we have

$$
\begin{aligned}
\left|\tilde{x}_{n}(t)-\tilde{x}(t)\right|= & \mid \mathcal{F}\left(t, x_{n}(t), \tilde{x}_{n}(t)\right)+\int_{0}^{t} \frac{(t-\xi)^{\sigma-1}}{\Gamma(\delta)} \mathcal{G}\left(\xi, x_{n}(\xi), \tilde{x}_{n}(\xi)\right) d \xi \\
& -\mathcal{F}(t, x(t), \tilde{x}(t))-\int_{0}^{t} \frac{(t-\xi)^{\sigma-1}}{\Gamma(\delta)} \mathcal{G}(\xi, x(\xi), \tilde{x}(\xi)) d \xi \mid \\
\leq & \left|\mathcal{F}\left(t, x_{n}(t), \tilde{x}_{n}(t)\right)-\mathcal{F}(t, x(t), \tilde{x}(t))\right|+\int_{0}^{t} \frac{(t-\xi)^{\sigma-1}}{\Gamma(\delta)}\left|\mathcal{G}\left(\xi, x_{n}(\xi), \tilde{x}_{n}(\xi)\right)-\mathcal{G}(\xi, x(\xi), \tilde{x}(\xi))\right| d \xi \\
\leq & \mathrm{M}_{1}\left|x_{n}(t)-x(t)\right|+\mathrm{N}_{1}\left|\tilde{x}_{n}(t)-\tilde{x}(t)\right|+\frac{t^{\sigma}}{\sigma \Gamma(\delta)}\left(\mathrm{M}_{2}\left|x_{n}(t)-x(t)\right|+\mathrm{N}_{2}\left|\tilde{x}_{n}(t)-\tilde{x}(t)\right|\right) .
\end{aligned}
$$

Then

$$
\left|\tilde{x}_{n}(t)-\tilde{x}(t)\right| \leq\left(\frac{\mathrm{M}_{1}}{1-\mathrm{N}_{1}-\mathrm{N}_{2} \frac{t^{\sigma}}{\sigma \Gamma(\delta)}}+\frac{\mathrm{M}_{2} \frac{t^{\sigma}}{\sigma \Gamma(\delta)}}{1-\mathrm{N}_{1}-\mathrm{N}_{2} \frac{t^{\sigma}}{\sigma \Gamma(\delta)}}\right)\left|x_{n}(t)-x(t)\right| .
$$

Thus, using hypothesis $\left[A_{1}\right]-\left[A_{3}\right]$ and (3.9), inequality (3.8) implies

$$
\begin{aligned}
\left|\left(\mathcal{T} x_{n}\right)(t)-(\mathcal{T} x)(t)\right| \leq & {\left[\left(\frac{(1+m) t^{\mathfrak{r}}}{\Gamma(\mathfrak{r}+1)}\right)\left(\frac{\mathrm{M}_{1}}{1-\mathrm{N}_{1}-\mathrm{N}_{2} \frac{t^{\sigma}}{\sigma \Gamma(\delta)}}+\frac{\mathrm{M}_{2} \frac{t^{\sigma}}{\sigma \Gamma(\delta)}}{1-\mathrm{N}_{1}-\mathrm{N}_{2} \frac{t^{\sigma}}{\sigma \Gamma(\delta)}}\right)\right.} \\
& \left.+m A_{I_{i}}+A_{\mathrm{h}}\right]\left|x_{n}(t)-x(t)\right|, i=1,2, \ldots, m .
\end{aligned}
$$

Since for each $t \in \mathcal{J}$, the sequence $x_{n} \rightarrow x$ as $n \rightarrow \infty$, hence by Lebesgue dominated convergence theorem, (3.8) implies that

$$
\left|\left(\mathcal{T} x_{n}\right)(t)-(\mathcal{T} x)(t)\right| \rightarrow 0 \text { as } n \rightarrow \infty
$$

Hence

$$
\left\|\mathcal{T} x_{n}-\mathcal{T} X\right\|_{\mathcal{M} C} \rightarrow 0 \text { as } n \rightarrow \infty
$$

So $\mathcal{T}$ is continuous on $\mathcal{J}$.

Now, we have to show that $\mathcal{T}$ is bounded in $\mathcal{M}$. So, for any $\wp>0$, there is $R_{\mathbf{E}}>0$, so that

$$
\mathbf{E}=\left\{x \in \mathcal{M}:\|x\|_{\mathcal{M}} \leq \wp\right\}
$$

then, we have

$$
\|\mathcal{T} X\|_{\mathcal{M}} \leq \mathrm{R}_{\mathrm{E}}
$$


For $t \in \mathcal{J}_{i}$, consider

$$
|(\mathcal{T} x)(t)| \leq \frac{1}{\Gamma(\mathfrak{r})} \int_{t_{i}}^{t}(t-\xi)^{\mathfrak{r}-1}|\tilde{x}(\xi)| d \xi+\sum_{i=1}^{m}\left[\frac{1}{\Gamma(\mathfrak{r})} \int_{t_{i-1}}^{t_{i}}\left(t_{i}-\xi\right)^{\mathfrak{r}-1}|\tilde{x}(\xi)| d \xi+\left|I_{i}\left(x\left(t_{i}\right)\right)\right|\right]+|\mathrm{h}(x)|, i=1,2, \ldots, m .
$$

Using $\left[A_{4}\right]$, we have

$$
\begin{aligned}
|\tilde{x}(t)| & \leq|\mathcal{F}(t, x(t), \tilde{x}(t))|+\int_{0}^{t} \frac{(t-\xi)^{\sigma-1}}{\Gamma(\delta)}|\mathcal{G}(\xi, x(\xi), \tilde{x}(\xi))| d \xi \\
& \leq a_{1}(t)+b_{1}(t)|x(t)|+c_{1}(t)|\tilde{x}(t)|+\frac{t^{\sigma}}{\sigma \Gamma(\delta)}\left(a_{2}(t)+b_{2}(t)|x(t)|+c_{2}(t)|\tilde{x}(t)|\right) \\
& \leq a_{1}^{\star}+b_{1}^{\star}\|x\|_{\mathcal{M}}+c_{1}^{\star}\|\tilde{x}\|_{\mathcal{M}}+\frac{\mathrm{T}^{\sigma}}{\sigma \Gamma(\delta)}\left(a_{2}^{\star}+b_{2}^{\star}\|x\|_{\mathcal{M}}+c_{2}^{\star}\|\tilde{x}\|_{\mathcal{M}}\right) .
\end{aligned}
$$

Therefore, we get

$$
|\tilde{x}(t)| \leq\|\tilde{x}\|_{\mathcal{M}} \leq \frac{a_{1}^{\star}+b_{1}^{\star}\|x\|_{\mathcal{M}}}{1-c_{1}^{\star}-c_{2}^{\star} \frac{T^{\sigma}}{\sigma \Gamma(\delta)}}+\frac{\mathrm{T}^{\sigma}}{\sigma \Gamma(\delta)} \frac{a_{2}^{\star}+b_{2}^{\star}\|x\|_{\mathcal{M}}}{1-c_{1}^{\star}-c_{2}^{\star} \frac{T^{\sigma}}{\sigma \Gamma(\delta)}}=\hbar .
$$

By using (3.11) and $\left[A_{4}\right]-\left[A_{6}\right]$, (3.10) becomes

$$
\begin{aligned}
|\mathcal{T} x(t)| & \leq \frac{\hbar}{\Gamma(\mathfrak{r})} \int_{t_{i}}^{t}(t-\xi)^{\mathfrak{r}-1} d \xi+\frac{\hbar}{\Gamma(\mathfrak{r})} \sum_{i=1}^{m} \int_{t_{i-1}}^{t_{i}}\left(t_{i}-\xi\right)^{\mathfrak{r}-1} d \xi+m(\mathcal{K}|x|+\mathcal{L})+\lambda \\
& \leq \frac{\hbar(1+m) t^{\mathfrak{r}}}{\Gamma(\mathfrak{r}+1)}+m\left(\mathcal{K}_{\wp}+\mathcal{L}\right)+\lambda=\eta .
\end{aligned}
$$

Thus

$$
\|\mathcal{T} x\|_{\mathcal{M}} \leq \eta
$$

Similarly, for $t \in \mathcal{J}_{0}$ we can verify that

$$
\|\mathcal{T} x\|_{\mathcal{M}} \leq \eta .
$$

Now we have to show that the operator $\mathcal{T}$ is equicontinuous in $\mathbf{E}$. Suppose $t_{1}, t_{2} \in \mathcal{J}_{i}, i=1,2, \ldots, m$ so that $0<t_{1}<t_{2}<\mathrm{T}$ and let $x \in \mathbf{E}$, then

$$
\begin{aligned}
\left|\mathcal{T} x\left(t_{2}\right)-\mathcal{T} x\left(t_{1}\right)\right| \leq & \frac{1}{\Gamma(\mathfrak{r})} \int_{t_{i}}^{t_{2}}\left(t_{2}-\xi\right)^{\mathfrak{r}-1}|\tilde{x}(\xi)| d \xi+\frac{1}{\Gamma(\mathfrak{r})} \int_{t_{i}}^{t_{1}}\left(t_{1}-\xi\right)^{\mathfrak{r}-1}|\tilde{x}(\xi)| d \xi \\
& +\sum_{0<t_{i}<t_{2}-t_{1}}\left[\frac{1}{\Gamma(\mathfrak{r})} \int_{t_{i-1}}^{t_{i}}\left(t_{i}-\xi\right)^{\mathfrak{r}-1}|\tilde{x}(\xi)| d \xi+\left|I_{i}\left(x\left(t_{i}\right)\right)\right|\right] \\
\leq & \frac{1}{\Gamma(\mathfrak{r})} \int_{t_{i}}^{t_{1}}\left[\left(t_{2}-\xi\right)^{\mathfrak{r}-1}-\left(t_{1}-\xi\right)^{\mathfrak{r}-1}\right]|\tilde{x}(\xi)| d \xi+\frac{1}{\Gamma(\mathfrak{r})} \int_{t_{1}}^{t_{2}}\left(t_{2}-\xi\right)^{\mathfrak{r}-1}|\tilde{x}(\xi)| d \xi \\
& +\sum_{0<t_{i}<t_{2}-t_{1}}\left[\frac{1}{\Gamma(\mathfrak{r})} \int_{t_{i-1}}^{t_{i}}\left(t_{i}-\xi\right)^{\mathfrak{r}-1}|\tilde{x}(\xi)| d \xi+\left|I_{i}\left(x\left(t_{i}\right)\right)\right|\right] .
\end{aligned}
$$

Obviously, the right-hand side of inequality (3.12) tends to zero as $t_{1} \rightarrow t_{2}$. Therefore,

$$
\left|\mathcal{T} x\left(t_{2}\right)-\mathcal{T} x\left(t_{1}\right)\right| \rightarrow 0 \text { as } t_{1} \rightarrow t_{2} .
$$


Similarly, we can show for $t \in \mathcal{J}_{0}$. Thus, $\mathcal{T}$ is equicontinuous and therefore completely continuous. Finally, we consider a set $\Omega \subset \mathcal{N}$, which is defined as

$$
\Omega=\{x \in \mathcal{M}: x=\mathcal{N} \mathcal{T} x, 0<\mathcal{M}<1\} .
$$

We need to prove that set $\Omega$ is bounded. Suppose $x \in \Omega$, so that

$$
x(t)=\aleph \mathcal{T} x(t), \text { where } 0<\aleph<1 .
$$

Then for each $t \in \mathcal{J}_{i}, i=1,2, \ldots, m$, we have

$$
\begin{aligned}
|x(t)| & =\left|\frac{\aleph}{\Gamma(\mathfrak{r})} \int_{t_{i}}^{t}(t-\xi)^{\mathfrak{r}-1} \tilde{x}(\xi) d \xi+\aleph \sum_{i=1}^{m}\left[\frac{1}{\Gamma(\mathfrak{r})} \int_{t_{i-1}}^{t_{i}}(t-\xi)^{\mathfrak{r}-1} \tilde{x}(\xi) d \xi+I_{i}\left(x\left(t_{i}\right)\right)\right]+\aleph \mathrm{h}(x)\right| \\
& \leq \frac{1}{\Gamma(\mathfrak{r})} \int_{t_{i}}^{t}(t-\xi)^{\mathfrak{r}-1}|\tilde{x}(\xi)| d \xi+\sum_{i=1}^{m}\left[\frac{1}{\Gamma(\mathfrak{r})} \int_{t_{i-1}}^{t_{i}}(t-\xi)^{\mathfrak{r}-1}|\tilde{x}(\xi)| d \xi+\left|I_{i}\left(x\left(t_{i}\right)\right)\right|\right]+|\mathrm{h}(x)| \\
& \leq \frac{(1+m) t^{\mathfrak{r}}}{\Gamma(\mathfrak{r}+1)}\|\tilde{x}\|_{\mathcal{M}}+m\left(\mathcal{K}_{\wp}+\mathcal{L}\right)+\lambda .
\end{aligned}
$$

Taking norm on both sides, we have $\|x\|_{\mathcal{M}} \leq Q$.

Also, for $t \in \mathcal{J}_{0}$, we can show that $\|x\|_{\mathcal{M}} \leq \mathcal{Q}$.

Hence, $\Omega$ is bounded. Thus, by Schaefer's fixed point theorem, $\mathcal{T}$ has at least one fixed point. So the considered problem (1.1) has at least one solution in $\mathcal{M}$.

The next result is based on Theorem 2.6 and concerned with the uniqueness of solution for (1.1).

Theorem 3.4. If the hypothesis $\left[A_{1}\right]-\left[A_{3}\right]$ along with the inequality

$$
\left[\left(\frac{(1+m) \mathrm{T}^{\mathfrak{r}}}{\Gamma(\mathfrak{r}+1)}\right)\left(\frac{\mathrm{M}_{1}}{1-\mathrm{N}_{1}-\mathrm{N}_{2} \frac{\mathrm{T}^{\sigma}}{\sigma \Gamma(\delta)}}+\frac{\mathrm{M}_{2} \frac{\mathrm{T}^{\sigma}}{\sigma \Gamma(\delta)}}{1-\mathrm{N}_{1}-\mathrm{N}_{2} \frac{\mathrm{T}^{\sigma}}{\sigma \Gamma(\delta)}}\right)+m A_{I_{i}}+A_{\mathrm{h}}\right]<1
$$

are satisfied, then problem (1.1) has a unique solution.

Proof. Suppose $x, \bar{x} \in \mathcal{M}$ and for $t \in \mathcal{J}_{i}, i=1,2, \ldots, m$, we have

$$
\begin{aligned}
|(\mathcal{T} x)(t)-(\mathcal{T} \bar{x})(t)| \leq & \frac{1}{\Gamma(\mathfrak{r})} \int_{t_{i}}^{t}(t-\xi)^{\mathfrak{r}-1}|\tilde{x}(\xi)-\bar{x}(\xi)| d \xi+\sum_{i=1}^{m} \frac{1}{\Gamma(\mathfrak{r})} \int_{t_{i-1}}^{t_{i}}\left(t_{i}-\xi\right)^{\mathfrak{r}-1}|\tilde{x}(\xi)-\overline{\tilde{x}}(\xi)| d \xi \\
& +\sum_{i=1}^{m}\left|I\left(x\left(t_{i}\right)\right)-I\left(\bar{x}\left(t_{i}\right)\right)\right|+|\mathrm{h}(x)-\mathrm{h}(\bar{x})|,
\end{aligned}
$$

where $\tilde{x}, \overline{\tilde{x}} \in \mathcal{M}$ are given by

$$
\tilde{x}(t)=\mathcal{F}(t, x(t), \tilde{x}(t))+\int_{0}^{t} \frac{(t-\xi)^{\sigma-1}}{\Gamma(\delta)} \mathcal{G}(\xi, x(\xi), \tilde{x}(\xi)) d \xi
$$

and

$$
\overline{\tilde{x}}(t)=\mathcal{F}(t, \bar{x}(t), \overline{\tilde{x}}(t))+\int_{0}^{t} \frac{(t-\xi)^{\sigma-1}}{\Gamma(\delta)} \mathcal{G}(\xi, \bar{x}(\xi), \bar{x}(\xi)) d \xi
$$


Using $\left[A_{1}\right]$, we have

$$
\begin{aligned}
|\tilde{x}(t)-\overline{\tilde{x}}(t)| & =\left|\mathcal{F}(t, x(t), \tilde{x}(t))+\int_{0}^{t} \frac{(t-\xi)^{\sigma-1}}{\Gamma(\delta)} \mathcal{G}(\xi, x(\xi), \tilde{x}(\xi)) d \xi-\mathcal{F}(t, \bar{x}(t), \overline{\tilde{x}}(t))-\int_{0}^{t} \frac{(t-\xi)^{\sigma-1}}{\Gamma(\delta)} \mathcal{G}(\xi, \bar{x}(\xi), \overline{\tilde{x}}(\xi)) d \xi\right| \\
& \leq|\mathcal{F}(t, x(t), \tilde{x}(t))-\mathcal{F}(t, \bar{x}(t), \overline{\tilde{x}}(t))|+\int_{0}^{t} \frac{(t-\xi)^{\sigma-1}}{\Gamma(\delta)}|\mathcal{G}(\xi, x(\xi), \tilde{x}(\xi))-\mathcal{G}(\xi, \bar{x}(\xi), \bar{x}(\xi))| d \xi \\
& \leq \mathrm{M}_{1}|x(t)-\bar{x}(t)|+\mathrm{N}_{1}|\tilde{x}(t)-\overline{\tilde{x}}(t)|+\frac{t^{\sigma}}{\sigma \Gamma(\delta)}\left(\mathrm{M}_{2}|x(t)-\bar{x}(t)|+\mathrm{N}_{2}|\tilde{x}(t)-\overline{\tilde{x}}(t)|\right) .
\end{aligned}
$$

Thus

$$
|\tilde{x}(t)-\overline{\tilde{x}}(t)| \leq\left(\frac{\mathrm{M}_{1}}{1-\mathrm{N}_{1}-\mathrm{N}_{2} \frac{t^{\sigma}}{\sigma \Gamma(\delta)}}+\frac{\mathrm{M}_{2} \frac{t^{\sigma}}{\sigma \Gamma(\delta)}}{1-\mathrm{N}_{1}-\mathrm{N}_{2} \frac{t^{\sigma}}{\sigma \Gamma(\delta)}}\right)|x(t)-\bar{x}(t)| .
$$

Thus, using hypothesis $\left[A_{1}\right]-\left[A_{3}\right]$ and (3.16), inequality (3.15) implies

$$
|(\mathcal{T} x)(t)-(\mathcal{T} \bar{x})(t)| \leq\left[\left(\frac{(1+m) t^{\mathfrak{r}}}{\Gamma(\mathfrak{r}+1)}\right)\left(\frac{\mathrm{M}_{1}}{1-\mathrm{N}_{1}-\mathrm{N}_{2} \frac{t^{\sigma}}{\sigma \Gamma(\delta)}}+\frac{\mathrm{M}_{2} \frac{t^{\sigma}}{\sigma \Gamma(\delta)}}{1-\mathrm{N}_{1}-\mathrm{N}_{2} \frac{t^{\sigma}}{\sigma \Gamma(\delta)}}\right)+m A_{I_{i}}+A_{\mathrm{h}}\right]|x(t)-\bar{x}(t)| .
$$

Now taking norm on both sides, we have

$$
\|\mathcal{T} x-\mathcal{T} \bar{x}\|_{\mathcal{M}} \leq\left[\left(\frac{(1+m) \mathrm{T}^{\mathfrak{r}}}{\Gamma(\mathfrak{r}+1)}\right)\left(\frac{\mathrm{M}_{1}}{1-\mathrm{N}_{1}-\mathrm{N}_{2} \frac{\mathrm{T}^{\sigma}}{\sigma \Gamma(\delta)}}+\frac{\mathrm{M}_{2} \frac{\mathrm{T}^{\sigma}}{\sigma \Gamma(\delta)}}{1-\mathrm{N}_{1}-\mathrm{N}_{2} \frac{\mathrm{T}^{\sigma}}{\sigma \Gamma(\delta)}}\right)+m A_{I_{i}}+A_{\mathrm{h}}\right]\|x-\bar{x}\|_{\mathcal{M}} .
$$

Similarly, for $x, \bar{x} \in \mathcal{M}$ and $t \in \mathcal{J}_{0}$, we get

$$
\|\mathcal{T} x-\mathcal{T} \bar{x}\|_{\mathcal{M}} \leq\left[\frac{\mathrm{T}^{\mathfrak{r}}}{\Gamma(\mathfrak{r}+1)}\left(\frac{\mathrm{M}_{1}}{1-\mathrm{N}_{1}-\mathrm{N}_{2} \frac{\mathrm{T}^{\sigma}}{\sigma \Gamma(\delta)}}+\frac{\mathrm{M}_{2} \frac{\mathrm{T}^{\sigma}}{\sigma \Gamma(\delta)}}{1-\mathrm{N}_{1}-\mathrm{N}_{2} \frac{\mathrm{T}^{\sigma}}{\sigma \Gamma(\delta)}}\right)+A_{\mathrm{h}}\right]\|x-\bar{x}\|_{\mathcal{M} \boldsymbol{\mathcal { C }}}
$$

Since,

$$
\begin{aligned}
{\left[\frac{\mathrm{T}^{\mathfrak{r}}}{\Gamma(\mathfrak{r}+1)}\left(\frac{\mathrm{M}_{1}}{1-\mathrm{N}_{1}-\mathrm{N}_{2} \frac{\mathrm{T}^{\sigma}}{\sigma \Gamma(\delta)}}+\frac{\mathrm{M}_{2} \frac{\mathrm{T}^{\sigma}}{\sigma \Gamma(\delta)}}{1-\mathrm{N}_{1}-\mathrm{N}_{2} \frac{T^{\sigma}}{\sigma \Gamma(\delta)}}\right)+A_{\mathrm{h}}\right] } \\
\leq\left[\left(\frac{(1+m) \mathrm{T}^{\mathfrak{r}}}{\Gamma(\mathfrak{r}+1)}\right)\left(\frac{\mathrm{M}_{1}}{1-\mathrm{N}_{1}-\mathrm{N}_{2} \frac{\mathrm{T}^{\sigma}}{\sigma \Gamma(\delta)}}+\frac{\mathrm{M}_{2} \frac{\mathrm{T}^{\sigma}}{\sigma \Gamma(\delta)}}{1-\mathrm{N}_{1}-\mathrm{N}_{2} \frac{T^{\sigma}}{\sigma \Gamma(\delta)}}\right)+m A_{I_{i}}+A_{\mathrm{h}}\right]<1 .
\end{aligned}
$$

Hence, $\mathcal{T}$ is a contraction. Which implies $\mathcal{T}$ has a unique fixed point, so the problem (1.1) has a unique solution.

After this, we consider a CS of nonlinear implicit FDEs with impulsive conditions of (1.2).

Theorem 3.5. The system:

$$
\left\{\begin{array}{l}
{ }^{c} D^{\mathfrak{r}} x(t)=\alpha(t), \forall t \in \mathcal{J}, \\
{ }^{c} D^{\mathfrak{s}} y(t)=\beta(t), \forall t \in \mathcal{J}, \\
x(0)=\mathrm{h}(x), \Delta x\left(t_{i}\right)=I_{i}\left(x\left(t_{i}\right)\right), \quad i=1,2, \ldots, m, \\
y(0)=\mathrm{g}(y), \Delta y\left(t_{j}\right)=I_{j}\left(y\left(t_{j}\right)\right), \quad j=1,2, \ldots, n,
\end{array}\right.
$$


has a solution $(x, y)$ if and only if

$$
x(t)=\left\{\begin{array}{l}
\frac{1}{\Gamma(\mathfrak{r})} \int_{0}^{t}(t-\xi)^{\mathfrak{r}-1} \alpha(\xi) d \xi+\mathrm{h}(x), t \in \mathcal{J}_{0}, \\
\frac{1}{\Gamma(\mathfrak{r})} \int_{t_{i}}^{t}(t-\xi)^{\mathfrak{r}-1} \alpha(\xi) d \xi \\
+\sum_{i=1}^{m}\left[\frac{1}{\Gamma(\mathfrak{r})} \int_{t_{i-1}}^{t_{i}}\left(t_{i}-\xi\right)^{\mathfrak{r}-1} \alpha(\xi) d \xi+I_{i}\left(x\left(t_{i}\right)\right)\right]+\mathrm{h}(x), t \in \mathcal{J}_{i}, i=1,2, \ldots, m
\end{array}\right.
$$

and

$$
y(t)=\left\{\begin{array}{l}
\frac{1}{\Gamma(\mathfrak{s})} \int_{0}^{t}(t-\xi)^{\mathfrak{s}-1} \beta(\xi) d \xi+\mathrm{g}(y), t \in \mathcal{J}_{0}, \\
\frac{1}{\Gamma(\mathfrak{s})} \int_{t_{j}}^{t}(t-\xi)^{\mathfrak{s}-1} \beta(\xi) d \xi \\
+\sum_{j=1}^{n}\left[\frac{1}{\Gamma(\mathfrak{s})} \int_{t_{j-1}}^{t_{j}}\left(t_{j}-\xi\right)^{\mathfrak{s}-1} \beta(\xi) d \xi+I_{j}\left(y\left(t_{j}\right)\right)\right]+\mathrm{g}(y), t \in \mathcal{J}_{j}, j=1,2, \ldots, n,
\end{array}\right.
$$

where

$$
\alpha(t)=\mathcal{F}\left(t, y(t),{ }^{c} D^{\mathfrak{r}} x(t)\right)+\int_{0}^{t} \frac{(t-\xi)^{\sigma-1}}{\Gamma(\delta)} \mathcal{G}\left(\xi, y(\xi),{ }^{c} D^{\mathfrak{r}} x(\xi)\right) d \xi
$$

and

$$
\beta(t)=\mathcal{F}^{\prime}\left(t, x(t),{ }^{c} D^{\mathfrak{s}} y(t)\right)+\int_{0}^{t} \frac{(t-\xi)^{\sigma-1}}{\Gamma(\delta)} \mathcal{G}^{\prime}\left(\xi, x(\xi),{ }^{c} D^{\mathfrak{s}} y(\xi)\right) d \xi
$$

Proof. The proof is similar as given in Theorem 3.1.

For $t_{i} \in \mathcal{J}$ so that $t_{1}<t_{2}<\cdots<t_{m}$ and $\mathcal{J}^{\prime}=\mathcal{J}-\left\{t_{1}, t_{2}, \ldots, t_{m}\right\}$ define the space $X=\{x: \mathcal{J} \rightarrow \mathbb{R} \mid x \in$ $\mathcal{C}\left(g^{\prime}\right)$, right limit $x\left(t_{i}^{+}\right)$and left limit $x\left(t_{i}^{-}\right)$exist and $\left.x\left(t_{i}^{-}\right)-x\left(t_{i}^{+}\right)=\Delta x\left(t_{i}\right), 1<i \leq m\right\}$.

Then, clearly $(X,\|\cdot\|)$ is a Banach space with norm $\|x\|=\max _{t \in \mathcal{J}}|x|$.

Similarly, for $t_{j} \in \mathcal{J}$ so that $t_{1}<t_{2}<\cdots<t_{n}$ and $\mathcal{J}^{\prime}=\mathcal{J}-\left\{t_{1}, t_{2}, \ldots, t_{n}\right\}$ define the space $y=\{y: \mathcal{J} \rightarrow \mathbb{R} \mid y \in$ $\mathcal{C}\left(\mathcal{J}^{\prime}\right)$, right limit $y\left(t_{j}^{+}\right)$left limit $y\left(t_{j}^{-}\right)$exist and $\left.y\left(t_{j}^{-}\right)-y\left(t_{j}^{+}\right)=\Delta y\left(t_{i}\right), 1<j \leq n\right\}$, which is a Banach space under the norm $\|y\|=\max _{t \in \mathcal{J}}|y|$.

Consequently, the product space $X \times y$ is a Banach space under the norm $\|(x, y)\|=\|x\|+\|y\|$ and $\|(x, y)\|=$ $\max \{\|x\|,\|y\|\}$.

Theorem 3.6. Let $\mathcal{F}, \mathcal{G}, \mathcal{F}^{\prime}, \mathcal{G}^{\prime}$ be continuous functions, then $(x, y) \in \mathcal{X} \times y$ is a solution of problem (1.2) if and only if

$$
x(t)=\frac{1}{\Gamma(\mathfrak{r})} \int_{t_{i}}^{t}(t-\xi)^{\mathfrak{r}-1} \alpha(\xi) d \xi+\sum_{i=1}^{m}\left[\frac{1}{\Gamma(\mathfrak{r})} \int_{t_{i-1}}^{t_{i}}\left(t_{i}-\xi\right)^{\mathfrak{r}-1} \alpha(\xi) d \xi+I_{i}\left(x\left(t_{i}\right)\right)\right]+\mathrm{h}(x), i=1,2, \ldots, m
$$


and

$$
y(t)=\frac{1}{\Gamma(\mathfrak{s})} \int_{t_{j}}^{t}(t-\xi)^{\mathfrak{s}-1} \beta(\xi) d \xi+\sum_{j=1}^{n}\left[\frac{1}{\Gamma(\mathfrak{s})} \int_{t_{j-1}}^{t_{j}}\left(t_{j}-\xi\right)^{\mathfrak{s}-1} \beta(\xi) d \xi+I_{j}\left(y\left(t_{j}\right)\right)\right]+\mathrm{g}(y), j=1,2, \ldots, n .
$$

Proof. If $(x, y)$ is a solution of the system (1.2), then it is a solution of (3.19). Conversely, let $(x, y)$ is a solution of (3.19), then

$$
\left\{\begin{array}{l}
{ }^{c} D^{\mathfrak{r}} x(t)=\mathcal{F}\left(t, y(t),{ }^{c} D^{\mathfrak{r}} x(t)\right)+\int_{0}^{t} \frac{(t-\xi)^{\sigma-1}}{\Gamma(\delta)} \mathcal{G}\left(\xi, y(\xi),{ }^{c} D^{\mathfrak{r}} x(\xi)\right) d \xi, t \in \mathcal{J}, t \neq t_{i} \text { for } i=1,2, \ldots, m, \\
{ }^{c} D^{\mathfrak{s}} y(t)=\mathcal{F}^{\prime}\left(t, x(t),{ }^{c} D^{\mathfrak{s}} y(t)\right)+\int_{0}^{t} \frac{(t-\xi)^{\sigma-1}}{\Gamma(\delta)} \mathcal{G}^{\prime}\left(\xi, x(\xi),{ }^{c} D^{\mathfrak{s}} y(\xi)\right) d \xi, t \in \mathcal{J}, t \neq t_{j} \text { for } j=1,2, \ldots, n, \\
x(0)=\mathrm{h}(x), \Delta x\left(t_{i}\right)=I_{i}\left(x\left(t_{i}\right)\right), \quad i=1,2, \ldots, m, \\
y(0)=\mathrm{g}(y), \Delta y\left(t_{j}\right)=I_{j}\left(y\left(t_{j}\right)\right), \quad j=1,2, \ldots, n .
\end{array}\right.
$$

Thus $(x, y)$ is a solution of (1.2).

For convenience, we use the following notations:

$$
\begin{aligned}
\tilde{x}(t) & =\mathcal{F}\left(t, y(t),{ }^{c} D^{\mathfrak{r}} x(t)\right)+\int_{0}^{t} \frac{(t-\xi)^{\sigma-1}}{\Gamma(\delta)} \mathcal{G}\left(\xi, y(\xi),{ }^{c} D^{\mathfrak{r}} x(\xi)\right) d \xi \\
& =\mathcal{F}(t, y(t), \tilde{x}(t))+\int_{0}^{t} \frac{(t-\xi)^{\sigma-1}}{\Gamma(\delta)} \mathcal{G}(\xi, y(\xi), \tilde{x}(\xi)) d \xi, \\
\tilde{y}(t) & =\mathcal{F}^{\prime}\left(t, x(t),{ }^{c} D^{\mathfrak{s}} y(t)\right)+\int_{0}^{t} \frac{(t-\xi)^{\sigma-1}}{\Gamma(\delta)} \mathcal{G}^{\prime}\left(\xi, x(\xi),{ }^{c} D^{\mathfrak{s}} y(\xi)\right) d \xi \\
& =\mathcal{F}^{\prime}(t, x(t), \tilde{y}(t))+\int_{0}^{t} \frac{(t-\xi)^{\sigma-1}}{\Gamma(\delta)} \mathcal{G}^{\prime}(\xi, x(\xi), \tilde{y}(\xi)) d \xi .
\end{aligned}
$$

The system (1.2) can be transformed into a fixed point problem.

Define an operators $\mathcal{T}_{\mathfrak{r}}, \mathcal{T}_{\mathfrak{s}}: X \times y \rightarrow X \times y$ by

$\mathcal{T}_{\mathfrak{r}}(x, y)(t)=\left\{\begin{array}{l}\frac{1}{\Gamma(\mathfrak{r})} \int_{0}^{t}(t-\xi)^{\mathfrak{r}-1} \tilde{x}(\xi) d \xi+\mathrm{h}(x), t \in \mathcal{J}_{0}, \\ \frac{1}{\Gamma(\mathfrak{r})} \int_{t_{i}}^{t}(t-\xi)^{\mathfrak{r}-1} \tilde{x}(\xi) d \xi+\sum_{i=1}^{m}\left[\frac{1}{\Gamma(\mathfrak{r})} \int_{t_{i-1}}^{t_{i}}\left(t_{i}-\xi\right)^{\mathfrak{r}-1} \tilde{x}(\xi) d \xi+I_{i}\left(x\left(t_{i}\right)\right)\right]+\mathrm{h}(x), t \in \mathcal{J}_{i}, i=1,2, \ldots, m,\end{array}\right.$

$\mathcal{T}_{\mathfrak{s}}(x, y)(t)=\left\{\begin{array}{l}\frac{1}{\Gamma(\mathfrak{s})} \int_{0}^{t}(t-\xi)^{\mathfrak{s}-1} \tilde{y}(\xi) d \xi+\mathrm{g}(y), t \in \mathcal{J}_{0}, \\ \frac{1}{\Gamma(\mathfrak{s})} \int_{t_{j}}^{t}(t-\xi)^{\mathfrak{s}-1} \tilde{y}(\xi) d \xi+\sum_{j=1}^{n}\left[\frac{1}{\Gamma(\mathfrak{s})} \int_{t_{j-1}}^{t_{j}}\left(t_{j}-\xi\right)^{\mathfrak{s}-1} \tilde{y}(\xi) d \xi+I_{j}\left(y\left(t_{j}\right)\right)\right]+\mathrm{g}(y), t \in \mathcal{J}_{j}, j=1,2, \ldots, n\end{array}\right.$

and $\mathcal{T}(x, y)(t)=\left(\mathcal{T}_{\mathfrak{r}}(x, y)(t), \mathcal{T}_{\mathfrak{s}}(x, y)(t)\right)$.

In the sequel we need the following hypotheses.

Assume that 
- $\left[H_{1}\right]$ there exist constants $\mathrm{M}_{1}>0$ and $0<\mathrm{N}_{1}<1$ so that for each $t \in \mathcal{J}$ and for all $u, \bar{u} \in \mathcal{X}$ and $w, \bar{w} \in \mathbb{R}$, we have

$$
|\mathcal{F}(t, u, w)-\mathcal{F}(t, \bar{u}, \bar{w})| \leq \mathrm{M}_{1}|u-\bar{u}|+\mathrm{N}_{1}|w-\bar{w}| ;
$$

Similarly, there exist constants $\mathrm{M}_{2}>0$ and $0<\mathrm{N}_{2}<1$ so that for each $t \in \mathcal{J}$ and for all $u, \bar{u} \in X$ and $w, \bar{w} \in \mathbb{R}$, we have

$$
|\mathcal{G}(t, u, w)-\mathcal{G}(t, \bar{u}, \bar{w})| \leq \mathrm{M}_{2}|u-\bar{u}|+\mathrm{N}_{2}|w-\bar{w}| ;
$$

- $\left[H_{2}\right]$ there exist constants $M_{1}^{\prime}>0$ and $0<N_{1}^{\prime}<1$ so that for each $t \in \mathcal{J}$ and for all $u, \bar{u} \in \mathcal{y}$ and $w, \bar{w} \in \mathbb{R}$, we have

$$
\left|\mathcal{F}^{\prime}(t, u, w)-\mathcal{F}^{\prime}(t, \bar{u}, \bar{w})\right| \leq \mathrm{M}_{1}^{\prime}|u-\bar{u}|+\mathrm{N}_{1}^{\prime}|w-\bar{w}| ;
$$

Similarly, there exist constants $\mathrm{M}_{2}^{\prime}>0$ and $0<\mathrm{N}_{2}^{\prime}<1$ so that for each $t \in \mathcal{J}$ and for all $u, \bar{u} \in \mathcal{y}$ and $w, \bar{w} \in \mathbb{R}$, we have

$$
\left|\mathcal{G}^{\prime}(t, u, w)-\mathcal{G}^{\prime}(t, \bar{u}, \bar{w})\right| \leq \mathrm{M}_{2}^{\prime}|u-\bar{u}|+\mathrm{N}_{2}^{\prime}|w-\bar{w}| ;
$$

- $\left[H_{3}\right]$ for any $u, \bar{u}, w, \bar{w} \in \mathcal{X} \times \bar{y}$, there exist constants $A_{I_{i}}, A_{I_{j}}>0$ so that

$$
\left|I_{i}\left(u\left(t_{i}\right)\right)-I_{i}\left(\bar{u}\left(t_{i}\right)\right)\right| \leq A_{I_{i}}\left|u\left(t_{i}\right)-\bar{u}\left(t_{i}\right)\right|,\left|I_{i}\left(w\left(t_{i}\right)\right)-I_{i}\left(\bar{w}\left(t_{i}\right)\right)\right| \leq A_{I_{i}}\left|w\left(t_{i}\right)-\bar{w}\left(t_{i}\right)\right| ;
$$

and

$$
\left|I_{j}\left(u\left(t_{j}\right)\right)-I_{j}\left(\bar{u}\left(t_{j}\right)\right)\right| \leq A_{I_{j}}\left|u\left(t_{j}\right)-\bar{u}\left(t_{j}\right)\right|,\left|I_{j}\left(w\left(t_{j}\right)\right)-I_{j}\left(\bar{w}\left(t_{j}\right)\right)\right| \leq A_{I_{j}}\left|w\left(t_{j}\right)-\bar{w}\left(t_{j}\right)\right| ;
$$

- $\left[H_{4}\right]$ for any $u, w \in \mathbb{R}$, there exist constants $A_{\mathrm{h}}, A_{\mathrm{g}}>0$ so that

$$
|\mathrm{h}(u)-\mathrm{h}(w)| \leq A_{\mathrm{h}}|u-w| ;
$$

and

$$
|\mathrm{g}(u)-\mathrm{g}(w)| \leq A_{\mathrm{g}}|u-w| ;
$$

- $\left[H_{5}\right]$ there exist $a_{1}, b_{1}, c_{1} \in X$ so that

$$
|\mathcal{F}(t, u(t), w(t))| \leq a_{1}(t)+b_{1}(t)|u(t)|+c_{1}(t)|w(t)|
$$

with $a_{1}^{\star}=\sup _{t \in \mathcal{J}} a_{1}(t), b_{1}^{\star}=\sup _{t \in \mathcal{J}} b_{1}(t)$ and $c_{1}^{\star}=\sup _{t \in \mathcal{J}} c_{1}(t)<1$; Similarly, there exist $a_{2}, b_{2}, c_{2} \in X$ so that

$$
|\mathcal{G}(t, u(t), w(t))| \leq a_{2}(t)+b_{2}(t)|u(t)|+c_{2}(t)|w(t)|
$$

with $a_{2}^{\star}=\sup _{t \in \mathcal{J}} a_{2}(t), b_{2}^{\star}=\sup _{t \in \mathcal{J}} b_{2}(t)$ and $c_{2}^{\star}=\sup _{t \in \mathcal{J}} c_{2}(t)<1$;

- $\left[H_{6}\right]$ there exist $l_{1}, m_{1}, n_{1} \in y$ so that

$$
\left|\mathcal{F}^{\prime}(t, u(t), w(t))\right| \leq l_{1}(t)+m_{1}(t)|u(t)|+n_{1}(t)|w(t)|
$$

with $l_{1}^{\star}=\sup _{t \in \mathcal{J}} l_{1}(t), m_{1}^{\star}=\sup _{t \in \mathcal{J}} m_{1}(t)$ and $n_{1}^{\star}=\sup _{t \in \mathcal{J}} n_{1}(t)<1$; Similarly, there exist $l_{2}, m_{2}, n_{2} \in y$ so that

$$
\left|\mathcal{G}^{\prime}(t, u(t), w(t))\right| \leq l_{2}(t)+m_{2}(t)|u(t)|+n_{2}(t)|w(t)|
$$

with $l_{2}^{\star}=\sup _{t \in \mathcal{J}} l_{2}(t), m_{2}^{\star}=\sup _{t \in \mathcal{J}} m_{2}(t)$ and $n_{2}^{\star}=\sup _{t \in \mathcal{J}} n_{2}(t)<1$;

- $\left[H_{7}\right]$ there exist constants $\lambda, \lambda^{\prime}>0$ so that

$$
|\mathrm{h}(u)| \leq \lambda, \forall u \in X
$$

and

$$
|\mathrm{g}(u)| \leq \lambda^{\prime}, \forall u \in \mathrm{y} ;
$$


- $\left[H_{8}\right]$ for each $u \in \mathbb{R}$, the function $I_{i}: \mathbb{R} \rightarrow \mathbb{R} ; i=1,2, \ldots, m$ are assumed to be continuous and for constants $\mathcal{K}, \mathcal{L}>0$, the inequality $\left|I_{i}\left(u\left(t_{i}\right)\right)\right| \leq \mathcal{K}|u(t)|+\mathcal{L}$, holds.

Similarly, for each $w \in \mathbb{R}$, the function $I_{j}: \mathbb{R} \rightarrow \mathbb{R} ; j=1,2, \ldots, n$ are assumed to be continuous and for constants $\mathcal{K}^{\prime}, \mathcal{L}^{\prime}>0$, the inequality $\left|I_{j}\left(w\left(t_{j}\right)\right)\right| \leq \mathcal{K}^{\prime}|w(t)|+\mathcal{L}^{\prime}$, holds.

$-\left[H_{9}\right]$ Define

$$
\Delta_{1}=\left[\left(\frac{(1+m) \mathrm{T}^{\mathfrak{r}}}{\Gamma(\mathfrak{r}+1)}\right)\left(\frac{\mathrm{M}_{1}}{1-\mathrm{N}_{1}-\mathrm{N}_{2} \frac{\mathrm{T}^{\sigma}}{\sigma \Gamma(\delta)}}+\frac{\mathrm{M}_{2} \frac{\mathrm{T}^{\sigma}}{\sigma \Gamma(\delta)}}{1-\mathrm{N}_{1}-\mathrm{N}_{2} \frac{\mathrm{T}^{\sigma}}{\sigma \Gamma(\delta)}}\right)+m A_{I_{i}}+A_{\mathrm{h}}\right]<1
$$

and

$$
\Delta_{2}=\left[\left(\frac{(1+n) \mathrm{T}^{\mathfrak{s}}}{\Gamma(\mathfrak{s}+1)}\right)\left(\frac{\mathrm{M}_{1}^{\prime}}{1-\mathrm{N}_{1}^{\prime}-\mathrm{N}_{2}^{\prime} \frac{\mathrm{T}^{\sigma}}{\sigma \Gamma(\delta)}}+\frac{\mathrm{M}_{2}^{\prime} \frac{\mathrm{T}^{\sigma}}{\sigma \Gamma(\delta)}}{1-\mathrm{N}_{1}^{\prime}-\mathrm{N}_{2}^{\prime} \frac{\mathrm{T}^{\sigma}}{\sigma \Gamma(\delta)}}\right)+n A_{I_{j}}+A_{\mathrm{g}}\right]<1 .
$$

Theorem 3.7. In addition to the hypothesis $\left[H_{3}\right]-\left[H_{8}\right]$, if $A_{\mathrm{h}}+m A_{I_{i}}<1$ and $A_{\mathrm{g}}+n A_{I_{j}}<1$, then system (1.2) has at least one solution.

Proof. Construct a closed ball $\mathcal{B} \subset \mathcal{X} \times y$ so that $\mathcal{B}=\{(x, y) \in \mathcal{X} \times \mathcal{y}:\|(x, y)\| \leq \mathbf{R}\}$. Split the operator $\mathcal{T}$ into two parts as $\mathcal{T}=\mathbb{F}+\mathbb{G}$ with $\mathbb{F}=\left(\mathbb{F}_{\mathfrak{r}}, \mathbb{F}_{\mathfrak{s}}\right)$ and $\mathbb{G}=\left(\mathbb{G}_{\mathfrak{r}}, \mathbb{G}_{\mathfrak{s}}\right)$, where

$$
\begin{aligned}
\mathbb{F}_{\mathfrak{r}}(x, y)(t) & =\frac{1}{\Gamma(\mathfrak{r})} \int_{t_{i}}^{t}(t-\xi)^{\mathfrak{r}-1} \tilde{x}(\xi) d \xi+\sum_{i=1}^{m} \frac{1}{\Gamma(\mathfrak{r})} \int_{t_{i-1}}^{t_{i}}\left(t_{i}-\xi\right)^{\mathfrak{r}-1} \tilde{x}(\xi) d \xi, i=1,2, \ldots, m, \\
\mathbb{F}_{\mathfrak{s}}(x, y)(t) & =\frac{1}{\Gamma(\mathfrak{s})} \int_{t_{j}}^{t}(t-\xi)^{\mathfrak{s}-1} \tilde{y}(\xi) d \xi+\sum_{j=1}^{n} \frac{1}{\Gamma(\mathfrak{s})} \int_{t_{j-1}}^{t_{j}}\left(t_{j}-\xi\right)^{\mathfrak{s}-1} \tilde{y}(\xi) d \xi, j=1,2, \ldots, n, \\
\mathbb{G}_{\mathfrak{r}}(x)(t) & =\mathrm{h}(x)+\sum_{i=1}^{m} I_{i}\left(x\left(t_{i}\right)\right), \\
\mathbb{G}_{\mathfrak{s}}(y)(t) & =\mathrm{g}(y)+\sum_{j=1}^{n} I_{j}\left(y\left(t_{j}\right)\right) .
\end{aligned}
$$

Clearly, $\mathcal{T}_{\mathfrak{r}}=\mathbb{F}_{\mathfrak{r}}+\mathbb{G}_{\mathfrak{r}}, \mathcal{T}_{\mathfrak{s}}=\mathbb{F}_{\mathfrak{s}}+\mathbb{G}_{\mathfrak{s}}$.

Now we show that $\mathcal{T}(x, y)(t)=\mathbb{F}(x, y)(t)+\mathbb{G}(x, y)(t) \in \mathcal{B}, \forall(x, y) \in \mathcal{B}$.

For any $(x, y) \in \mathcal{B}$, consider

$$
\left|\mathcal{T}_{\mathfrak{r}} x(t)\right| \leq \frac{1}{\Gamma(\mathfrak{r})} \int_{t_{i}}^{t}(t-\xi)^{\mathfrak{r}-1}|\tilde{x}(\xi)| d \xi+\sum_{i=1}^{m} \frac{1}{\Gamma(\mathfrak{r})} \int_{t_{i-1}}^{t_{i}}\left(t_{i}-\xi\right)^{\mathfrak{r}-1}|\tilde{x}(\xi)| d \xi+\sum_{i=1}^{m}\left|I_{i}\left(x\left(t_{i}\right)\right)\right|+|\mathrm{h}(x)| .
$$

By $\left[H_{5}\right]$, for $t \in \mathcal{J}_{i}, i=1,2, \ldots, m$, we have

$$
\begin{aligned}
|\tilde{x}(t)| & \leq|\mathcal{F}(t, x(t), \tilde{x}(t))|+\int_{0}^{t} \frac{(t-\xi)^{\sigma-1}}{\Gamma(\delta)}|\mathcal{G}(\xi, x(\xi), \tilde{x}(\xi))| d \xi \\
& \leq a_{1}(t)+b_{1}(t)|x(t)|+c_{1}(t)|\tilde{x}(t)|+\frac{t^{\sigma}}{\sigma \Gamma(\delta)}\left(a_{2}(t)+b_{2}(t)|x(t)|+c_{2}(t)|\tilde{x}(t)|\right) \\
& \leq a_{1}^{\star}+b_{1}^{\star}\|x\| x+c_{1}^{\star}\|\tilde{x}\| x+\frac{\mathrm{T}^{\sigma}}{\sigma \Gamma(\delta)}\left(a_{2}^{\star}+b_{2}^{\star}\|x\| x+c_{2}^{\star}\|\tilde{x}\| x\right) .
\end{aligned}
$$

Therefore, we get

$$
|\tilde{x}(t)| \leq\|\tilde{x}\|_{x} \leq \frac{a_{1}^{\star}+b_{1}^{\star}\|x\|_{x}}{1-c_{1}^{\star}-c_{2}^{\star} \frac{\mathrm{T}^{\sigma}}{\sigma \Gamma(\delta)}}+\frac{\mathrm{T}^{\sigma}}{\sigma \Gamma(\delta)} \frac{a_{2}^{\star}+b_{2}^{\star}\|x\|_{x}}{1-c_{1}^{\star}-c_{2}^{\star} \frac{\mathrm{T}^{\sigma}}{\sigma \Gamma(\delta)}}=\hbar .
$$


By using (3.21), $\left[H_{7}\right]$ and $\left[H_{8}\right]$, (3.20) becomes

$$
\begin{aligned}
\left|\mathcal{T}_{\mathfrak{r}} x(t)\right| & \leq \frac{\hbar}{\Gamma(\mathfrak{r})} \int_{t_{i}}^{t}(t-\xi)^{\mathfrak{r}-1} d \xi+\frac{\hbar}{\Gamma(\mathfrak{r})} \sum_{i=1}^{m} \int_{t_{i-1}}^{t_{i}}\left(t_{i}-\xi\right)^{\mathfrak{r}-1} d \xi+m(\mathcal{K}|x|+\mathcal{L})+\lambda \\
& \leq \frac{\hbar(1+m) t^{\mathfrak{r}}}{\Gamma(\mathfrak{r}+1)}+m\left(\mathcal{K}_{\wp}+\mathcal{L}\right)+\lambda=\eta .
\end{aligned}
$$

Thus

$$
\left\|\mathcal{T}_{\mathfrak{r}} x\right\|_{x} \leq \eta
$$

Similarly, for $t \in \mathcal{J}_{0}$ we can verify that

$$
\left\|\mathcal{T}_{\mathfrak{r}} x\right\|_{x} \leq \eta
$$

In the similar manner, we have

$$
\left|\mathcal{T}_{\mathfrak{r}} y(t)\right| \leq \frac{1}{\Gamma(\mathfrak{r})} \int_{t_{i}}^{t}(t-\xi)^{\mathfrak{r}-1}|\tilde{x}(\xi)| d \xi+\sum_{i=1}^{m} \frac{1}{\Gamma(\mathfrak{r})} \int_{t_{i-1}}^{t_{i}}\left(t_{i}-\xi\right)^{\mathfrak{r}-1}|\tilde{x}(\xi)| d \xi+\sum_{i=1}^{m}\left|I_{i}\left(y\left(t_{i}\right)\right)\right|+|\mathrm{h}(y)| .
$$

By $\left[H_{5}\right]$, for $t \in \mathcal{J}_{i}, i=1,2, \ldots, m$, we have

$$
\begin{aligned}
|\tilde{x}(t)| & \leq|\mathcal{F}(t, x(t), \tilde{x}(t))|+\int_{0}^{t} \frac{(t-\xi)^{\sigma-1}}{\Gamma(\delta)}|\mathcal{G}(\xi, x(\xi), \tilde{x}(\xi))| d \xi \\
& \leq a_{1}(t)+b_{1}(t)|x(t)|+c_{1}(t)|\tilde{x}(t)|+\frac{t^{\sigma}}{\sigma \Gamma(\delta)}\left(a_{2}(t)+b_{2}(t)|x(t)|+c_{2}(t)|\tilde{x}(t)|\right) \\
& \leq a_{1}^{\star}+b_{1}^{\star}\|x\| x+c_{1}^{\star}\|\tilde{x}\| x+\frac{\mathrm{T}^{\sigma}}{\sigma \Gamma(\delta)}\left(a_{2}^{\star}+b_{2}^{\star}\|x\| x+c_{2}^{\star}\|\tilde{x}\| x\right) .
\end{aligned}
$$

Therefore, we get

$$
|\tilde{x}(t)| \leq\|\tilde{x}\|_{X} \leq \frac{a_{1}^{\star}+b_{1}^{\star}\|x\|_{x}}{1-c_{1}^{\star}-c_{2}^{\star} \frac{\mathrm{T}^{\sigma}}{\sigma \Gamma(\delta)}}+\frac{\mathrm{T}^{\sigma}}{\sigma \Gamma(\delta)} \frac{a_{2}^{\star}+b_{2}^{\star}\|x\|_{x}}{1-c_{1}^{\star}-c_{2}^{\star} \frac{\mathrm{T}^{\sigma}}{\sigma \Gamma(\delta)}}=\hbar .
$$

By using (3.23) and $\left[H_{4}\right]-\left[H_{6}\right]$, (3.22) becomes

$$
\begin{aligned}
\left|\mathcal{T}_{\mathfrak{r}} y(t)\right| & \leq \frac{\hbar}{\Gamma(\mathfrak{r})} \int_{t_{i}}^{t}(t-\xi)^{\mathfrak{r}-1} d \xi+\frac{\hbar}{\Gamma(\mathfrak{r})} \sum_{i=1}^{m} \int_{t_{i-1}}^{t_{i}}\left(t_{i}-\xi\right)^{\mathfrak{r}-1} d \xi+m(\mathcal{K}|x|+\mathcal{L})+\lambda \\
& \leq \frac{\hbar(1+m) t^{\mathfrak{r}}}{\Gamma(\mathfrak{r}+1)}+m\left(\mathcal{K}_{\wp}+\mathcal{L}\right)+\lambda=\eta .
\end{aligned}
$$

Thus

$$
\left\|\mathcal{T}_{\mathfrak{r}} y\right\|_{X} \leq \eta
$$

Similarly, for $t \in \mathcal{J}_{0}$ we can verify that

$$
\left\|\mathcal{T}_{\mathfrak{r}} y\right\|_{x} \leq \eta
$$

Hence

$$
\left\|\mathcal{T}_{\mathfrak{r}}(x, y)\right\|_{x} \leq \eta
$$

Now, for any $(x, y) \in \mathcal{B}$, consider

$$
\left|\mathcal{T}_{\mathfrak{s}} x(t)\right| \leq \frac{1}{\Gamma(\mathfrak{s})} \int_{t_{j}}^{t}(t-\xi)^{\mathfrak{s}-1}|\tilde{y}(\xi)| d \xi+\sum_{j=1}^{n} \frac{1}{\Gamma(\mathfrak{s})} \int_{t_{j-1}}^{t_{j}}\left(t_{j}-\xi\right)^{\mathfrak{s}-1}|\tilde{y}(\xi)| d \xi+\sum_{j=1}^{n}\left|I_{j}\left(x\left(t_{j}\right)\right)\right|+|\mathrm{g}(x)| .
$$


By $\left[H_{6}\right]$, for $t \in \mathcal{J}_{j}, j=1,2, \ldots, n$, we have

$$
\begin{aligned}
|\tilde{y}(t)| & \leq\left|\mathcal{F}^{\prime}(t, x(t), \tilde{y}(t))\right|+\int_{0}^{t} \frac{(t-\xi)^{\sigma-1}}{\Gamma(\delta)}\left|\mathcal{G}^{\prime}(\xi, x(\xi), \tilde{y}(\xi))\right| d \xi \\
& \leq l_{1}(t)+m_{1}(t)|x(t)|+n_{1}(t)|\tilde{y}(t)|+\frac{t^{\sigma}}{\sigma \Gamma(\delta)}\left(l_{2}(t)+m_{2}(t)|x(t)|+n_{2}(t)|\tilde{y}(t)|\right) \\
& \leq l_{1}^{\star}+m_{1}^{\star}\|x\|_{y}+n_{1}^{*}\|\tilde{y}\|_{y}+\frac{\mathrm{T}^{\sigma}}{\sigma \Gamma(\delta)}\left(l_{2}^{\star}+m_{2}^{\star}\|x\|_{y}+n_{2}^{\star}\|\tilde{y}\|_{y}\right) .
\end{aligned}
$$

Therefore, we get

$$
|\tilde{y}(t)| \leq\|\tilde{y}\|_{y} \leq \frac{l_{1}^{\star}+m_{1}^{\star}\|x\|_{y}}{1-n_{1}^{\star}-n_{2}^{\star} \frac{\mathrm{T}^{\sigma}}{\sigma \Gamma(\delta)}}+\frac{\mathrm{T}^{\sigma}}{\sigma \Gamma(\delta)} \frac{l_{2}^{\star}+m_{2}^{\star}\|x\|_{y}}{1-n_{1}^{\star}-n_{2}^{\star} \frac{\mathrm{T}^{\sigma}}{\sigma \Gamma(\delta)}}=\hbar .
$$

By using (3.25), $\left[H_{7}\right]$ and $\left[H_{8}\right]$, (3.24) becomes

$$
\begin{aligned}
\left|\mathcal{T}_{\mathfrak{s}} x(t)\right| & \leq \frac{\hbar}{\Gamma(\mathfrak{s})} \int_{t_{j}}^{t}(t-\xi)^{\mathfrak{s}-1} d \xi+\frac{\hbar}{\Gamma(\mathfrak{s})} \sum_{j=1}^{n} \int_{t_{j-1}}^{t_{j}}\left(t_{j}-\xi\right)^{\mathfrak{s}-1} d \xi+n\left(\mathcal{K}^{\prime}|x|+\mathcal{L}^{\prime}\right)+\lambda^{\prime} \\
& \leq \frac{\hbar(1+n) t^{\mathfrak{s}}}{\Gamma(\mathfrak{s}+1)}+n\left(\mathcal{K}^{\prime} \wp+\mathcal{L}^{\prime}\right)+\lambda^{\prime}=\eta^{\star} .
\end{aligned}
$$

Thus

$$
\left\|\mathcal{T}_{\mathfrak{s}}(x)\right\| y \leq \eta^{\star}
$$

In the similar manner, we have

$$
\left|\mathcal{T}_{\mathfrak{s}} y(t)\right| \leq \frac{1}{\Gamma(\mathfrak{s})} \int_{t_{j}}^{t}(t-\xi)^{\mathfrak{s}-1}|\tilde{y}(\xi)| d \xi+\sum_{j=1}^{n} \frac{1}{\Gamma(\mathfrak{s})} \int_{t_{j-1}}^{t_{j}}\left(t_{j}-\xi\right)^{\mathfrak{s}-1}|\tilde{y}(\xi)| d \xi+\sum_{j=1}^{n}\left|I_{j}\left(y\left(t_{j}\right)\right)\right|+|g(y)| .
$$

By $\left[H_{6}\right]$, for $t \in \mathcal{J}_{j}, j=1,2, \ldots, n$, we have

$$
\begin{aligned}
|\tilde{y}(t)| & \leq\left|\mathcal{F}^{\prime}(t, x(t), \tilde{y}(t))\right|+\int_{0}^{t} \frac{(t-\xi)^{\sigma-1}}{\Gamma(\delta)}\left|\mathcal{G}^{\prime}(\xi, x(\xi), \tilde{y}(\xi))\right| d \xi \\
& \leq l_{1}(t)+m_{1}(t)|x(t)|+n_{1}(t)|\tilde{y}(t)|+\frac{t^{\sigma}}{\sigma \Gamma(\delta)}\left(l_{2}(t)+m_{2}(t)|x(t)|+n_{2}(t)|\tilde{y}(t)|\right) \\
& \leq l_{1}^{\star}+m_{1}^{*}\|x\| y+n_{1}^{*}\|\tilde{y}\|_{y}+\frac{\mathrm{T}^{\sigma}}{\sigma \Gamma(\delta)}\left(l_{2}^{\star}+m_{2}^{\star}\|x\|_{y}+n_{2}^{*}\|\tilde{y}\|_{y}\right) .
\end{aligned}
$$

Therefore, we get

$$
|\tilde{y}(t)| \leq\|\tilde{y}\|_{y} \leq \frac{l_{1}^{\star}+m_{1}^{\star}\|x\|_{y}}{1-n_{1}^{\star}-n_{2}^{\star} \frac{\mathrm{T}^{\sigma}}{\sigma \Gamma(\delta)}}+\frac{\mathrm{T}^{\sigma}}{\sigma \Gamma(\delta)} \frac{l_{2}^{\star}+m_{2}^{\star}\|x\|_{y}}{1-n_{1}^{\star}-n_{2}^{\star} \frac{\mathrm{T}^{\sigma}}{\sigma \Gamma(\delta)}}=\hbar .
$$

By using (3.27), $\left[H_{7}\right]$ and $\left[H_{8}\right]$, (3.26) becomes

$$
\begin{aligned}
\left|\mathcal{T}_{\mathfrak{s}} y(t)\right| & \leq \frac{\hbar}{\Gamma(\mathfrak{s})} \int_{t_{j}}^{t}(t-\xi)^{\mathfrak{s}-1} d \xi+\frac{\hbar}{\Gamma(\mathfrak{s})} \sum_{j=1}^{n} \int_{t_{j-1}}^{t_{j}}\left(t_{j}-\xi\right)^{\mathfrak{s}-1} d \xi+n\left(\mathcal{K}^{\prime}|x|+\mathcal{L}^{\prime}\right)+\lambda^{\prime} \\
& \leq \frac{\hbar(1+n) t^{\mathfrak{s}}}{\Gamma(\mathfrak{s}+1)}+n\left(\mathcal{K}_{\wp}^{\prime} \wp+\mathcal{L}^{\prime}\right)+\lambda^{\prime}=\eta^{\star} .
\end{aligned}
$$

Thus

$$
\left\|\mathcal{T}_{\mathfrak{s}}(y)\right\|_{y} \leq \eta^{\star}
$$

Hence

$$
\left\|\mathcal{T}_{\mathfrak{s}}(x, y)\right\| y \leq \eta^{*}
$$


Thus

$$
\|\mathcal{T}(x, y)\|_{x \times y} \leq\left\|\mathcal{T}_{\mathfrak{r}}(x, y)+\mathcal{T}_{\mathfrak{s}}(x, y)\right\|_{x \times y} \leq \eta+\eta^{\star}=\mathbf{R} .
$$

Which implies that $\mathcal{T}(\mathcal{B}) \subseteq \mathcal{B}$.

Now we show that $\mathbb{G}$ is contraction.

For any $(x, y),(\bar{x}, \bar{y}) \in \mathcal{B}$, we have

$$
\begin{aligned}
\left|\mathbb{G}_{\mathfrak{r}}(x)-\mathbb{G}_{\mathfrak{r}}(\bar{x})\right| & \leq|\mathrm{h}(x)-\mathrm{h}(\bar{x})|+\sum_{i=1}^{m}\left|I_{i}\left(x\left(t_{i}\right)\right)-I_{i}\left(\bar{x}\left(t_{i}\right)\right)\right| \\
& \leq A_{\mathrm{h}}|x-\bar{x}|+m A_{I_{i}}|x-\bar{x}|
\end{aligned}
$$

and

$$
\begin{aligned}
\left|\mathbb{G}_{\mathfrak{s}}(y)-\mathbb{G}_{\mathfrak{s}}(\bar{y})\right| & \leq|\mathrm{g}(y)-\mathrm{g}(\bar{y})|+\sum_{j=1}^{n}\left|I_{j}\left(y\left(t_{j}\right)\right)-I_{j}\left(\bar{y}\left(t_{j}\right)\right)\right| \\
& \leq A_{\mathfrak{g}}|y-\bar{y}|+n A_{I_{j}}|y-\bar{y}| .
\end{aligned}
$$

The contraction of $\mathbb{G}$ follows from the assumption that $A_{\mathrm{h}}+m A_{I_{i}}<1$ and $A_{\mathrm{g}}+n A_{I_{j}}<1$.

Next $\mathbb{F}=\left(\mathbb{F}_{\mathfrak{r}}+\mathbb{F}_{\mathfrak{s}}\right)$ is compact. The continuity of $\mathbb{F}$ follows from the continuity of $\mathcal{F}, \mathcal{G}, \mathcal{F}^{\prime}, \mathcal{G}^{\prime}$. For $(x, y) \in \mathcal{B}$, we have

$$
\begin{aligned}
\left|\mathbb{F}_{\mathfrak{r}} x(t)\right| & =\left|\frac{1}{\Gamma(\mathfrak{r})} \int_{t_{i}}^{t}(t-\xi)^{\mathfrak{r}-1} \tilde{x}(\xi) d \xi+\sum_{i=1}^{m} \frac{1}{\Gamma(\mathfrak{r})} \int_{t_{i-1}}^{t_{i}}\left(t_{i}-\xi\right)^{\mathfrak{r}-1} \tilde{x}(\xi) d \xi\right| \\
& \leq \frac{1}{\Gamma(\mathfrak{r})} \int_{t_{i}}^{t}(t-\xi)^{\mathfrak{r}-1}|\tilde{x}(\xi)| d \xi+\sum_{i=1}^{m} \frac{1}{\Gamma(\mathfrak{r})} \int_{t_{i-1}}^{t_{i}}\left(t_{i}-\xi\right)^{\mathfrak{r}-1}|\tilde{x}(\xi)| d \xi
\end{aligned}
$$

By $\left[H_{5}\right]$, for $t \in \mathcal{J}_{i}, i=1,2, \ldots, m$, we have

$$
\begin{aligned}
|\tilde{x}(t)| & \leq|\mathcal{F}(t, x(t), \tilde{x}(t))|+\int_{0}^{t} \frac{(t-\xi)^{\sigma-1}}{\Gamma(\delta)}|\mathcal{G}(\xi, x(\xi), \tilde{x}(\xi))| d \xi \\
& \leq a_{1}(t)+b_{1}(t)|x(t)|+c_{1}(t)|\tilde{x}(t)|+\frac{t^{\sigma}}{\sigma \Gamma(\delta)}\left(a_{2}(t)+b_{2}(t)|x(t)|+c_{2}(t)|\tilde{x}(t)|\right) \\
& \leq a_{1}^{*}+b_{1}^{*}\|x\|_{x}+c_{1}^{*}\|\tilde{x}\|_{x}+\frac{\mathrm{T}^{\sigma}}{\sigma \Gamma(\delta)}\left(a_{2}^{\star}+b_{2}^{*}\|x\|_{x}+c_{2}^{*}\|\tilde{x}\|_{x}\right) .
\end{aligned}
$$

Therefore, we get

$$
|\tilde{x}(t)| \leq\|\tilde{x}\|_{x} \leq \frac{a_{1}^{\star}+b_{1}^{\star}\|x\|_{x}}{1-c_{1}^{\star}-c_{2}^{\star} \frac{\mathrm{T}^{\sigma}}{\sigma \Gamma(\delta)}}+\frac{\mathrm{T}^{\sigma}}{\sigma \Gamma(\delta)} \frac{a_{2}^{\star}+b_{2}^{\star}\|x\|_{x}}{1-c_{1}^{\star}-c_{2}^{\star} \frac{\mathrm{T}^{\sigma}}{\sigma \Gamma(\delta)}}=\hbar .
$$

Using (3.29) in (3.28) and after simplification, we get

$$
\left\|\mathbb{F}_{\mathfrak{r}} x\right\|_{x} \leq \eta_{1} .
$$

In the similar manner, we have

$$
\begin{aligned}
\left|\mathbb{F}_{\mathfrak{r}} y(t)\right| & =\left|\frac{1}{\Gamma(\mathfrak{r})} \int_{t_{i}}^{t}(t-\xi)^{\mathfrak{r}-1} \tilde{x}(\xi) d \xi+\sum_{i=1}^{m} \frac{1}{\Gamma(\mathfrak{r})} \int_{t_{i-1}}^{t_{i}}\left(t_{i}-\xi\right)^{\mathfrak{r}-1} \tilde{x}(\xi) d \xi\right| \\
& \leq \frac{1}{\Gamma(\mathfrak{r})} \int_{t_{i}}^{t}(t-\xi)^{\mathfrak{r}-1}|\tilde{x}(\xi)| d \xi+\sum_{i=1}^{m} \frac{1}{\Gamma(\mathfrak{r})} \int_{t_{i-1}}^{t_{i}}\left(t_{i}-\xi\right)^{\mathfrak{r}-1}|\tilde{x}(\xi)| d \xi
\end{aligned}
$$


By $\left[H_{5}\right]$, for $t \in \mathcal{J}_{i}, i=1,2, \ldots, m$, we have

$$
\begin{aligned}
|\tilde{x}(t)| & \leq|\mathcal{F}(t, x(t), \tilde{x}(t))|+\int_{0}^{t} \frac{(t-\xi)^{\sigma-1}}{\Gamma(\delta)}|\mathcal{G}(\xi, x(\xi), \tilde{x}(\xi))| d \xi \\
& \leq a_{1}(t)+b_{1}(t)|x(t)|+c_{1}(t)|\tilde{x}(t)|+\frac{t^{\sigma}}{\sigma \Gamma(\delta)}\left(a_{2}(t)+b_{2}(t)|x(t)|+c_{2}(t)|\tilde{x}(t)|\right) \\
& \leq a_{1}^{*}+b_{1}^{*}\|x\|_{x}+c_{1}^{*}\|\tilde{x}\|_{x}+\frac{\mathrm{T}^{\sigma}}{\sigma \Gamma(\delta)}\left(a_{2}^{*}+b_{2}^{*}\|x\|_{x}+c_{2}^{*}\|\tilde{x}\|_{x}\right) .
\end{aligned}
$$

Therefore, we get

$$
|\tilde{x}(t)| \leq\|\tilde{x}\|_{x} \leq \frac{a_{1}^{\star}+b_{1}^{\star}\|x\|_{x}}{1-c_{1}^{\star}-c_{2}^{\star} \frac{\mathrm{T}^{\sigma}}{\sigma \Gamma(\delta)}}+\frac{\mathrm{T}^{\sigma}}{\sigma \Gamma(\delta)} \frac{a_{2}^{\star}+b_{2}^{\star}\|x\|_{x}}{1-c_{1}^{\star}-c_{2}^{\star} \frac{\mathrm{T}^{\sigma}}{\sigma \Gamma(\delta)}}=\hbar .
$$

Using (3.31) in (3.30) and after simplification, we get

$$
\left\|\mathbb{F}_{\mathfrak{r}} y\right\|_{x} \leq \eta_{1} .
$$

Hence

$$
\left\|\mathbb{F}_{\mathfrak{r}}(x, y)\right\|_{x} \leq \eta_{1}
$$

Now for any $(x, y) \in \mathcal{B}$, we have

$$
\begin{aligned}
\left|\mathbb{F}_{\mathfrak{s}} x(t)\right| & =\left|\frac{1}{\Gamma(\mathfrak{s})} \int_{t_{j}}^{t}(t-\xi)^{\mathfrak{s}-1} \tilde{y}(\xi) d \xi+\sum_{j=1}^{n} \frac{1}{\Gamma(\mathfrak{s})} \int_{t_{j-1}}^{t_{j}}\left(t_{j}-\xi\right)^{\mathfrak{s}-1} \tilde{y}(\xi) d \xi\right| \\
& \leq \frac{1}{\Gamma(\mathfrak{s})} \int_{t_{j}}^{t}(t-\xi)^{\mathfrak{s}-1}|\tilde{y}(\xi)| d \xi+\sum_{j=1}^{n} \frac{1}{\Gamma(\mathfrak{s})} \int_{t_{j-1}}^{t_{j}}\left(t_{j}-\xi\right)^{\mathfrak{s}-1}|\tilde{y}(\xi)| d \xi
\end{aligned}
$$

By $\left[H_{6}\right]$, for $t \in \mathcal{J}_{j}, j=1,2, \ldots, n$, we have

$$
\begin{aligned}
|\tilde{y}(t)| & \leq\left|\mathcal{F}^{\prime}(t, x(t), \tilde{y}(t))\right|+\int_{0}^{t} \frac{(t-\xi)^{\sigma-1}}{\Gamma(\delta)}\left|\mathcal{G}^{\prime}(\xi, x(\xi), \tilde{y}(\xi))\right| d \xi \\
& \leq l_{1}(t)+m_{1}(t)|x(t)|+n_{1}(t)|\tilde{y}(t)|+\frac{t^{\sigma}}{\sigma \Gamma(\delta)}\left(l_{2}(t)+m_{2}(t)|x(t)|+n_{2}(t)|\tilde{y}(t)|\right) \\
& \leq l_{1}^{\star}+m_{1}^{\star}\|x\|_{y}+n_{1}^{\star}\|\tilde{y}\|_{y}+\frac{\mathrm{T}^{\sigma}}{\sigma \Gamma(\delta)}\left(l_{2}^{\star}+m_{2}^{\star}\|x\|_{y}+n_{2}^{\star}\|\tilde{y}\|_{y}\right) .
\end{aligned}
$$

Therefore, we get

$$
|\tilde{y}(t)| \leq\|\tilde{y}\|_{y} \leq \frac{l_{1}^{\star}+m_{1}^{\star}\|x\|_{y}}{1-n_{1}^{\star}-n_{2}^{\star} \frac{\mathrm{T}^{\sigma}}{\sigma \Gamma(\delta)}}+\frac{\mathrm{T}^{\sigma}}{\sigma \Gamma(\delta)} \frac{l_{2}^{\star}+m_{2}^{\star}\|x\|_{y}}{1-n_{1}^{\star}-n_{2}^{\star} \frac{\mathrm{T}^{\sigma}}{\sigma \Gamma(\delta)}}=\hbar .
$$

Using (3.33) in (3.32) and after simplification, we get

$$
\left\|\mathbb{F}_{\mathfrak{s}} x\right\| \leq \eta_{2} .
$$

In the similar manner, we have

$$
\begin{aligned}
\left|\mathbb{F}_{\mathfrak{s}} y(t)\right| & =\left|\frac{1}{\Gamma(\mathfrak{s})} \int_{t_{j}}^{t}(t-\xi)^{\mathfrak{s}-1} \tilde{y}(\xi) d \xi+\sum_{j=1}^{n} \frac{1}{\Gamma(\mathfrak{s})} \int_{t_{j-1}}^{t_{j}}\left(t_{j}-\xi\right)^{\mathfrak{s}-1} \tilde{y}(\xi) d \xi\right| \\
& \leq \frac{1}{\Gamma(\mathfrak{s})} \int_{t_{j}}^{t}(t-\xi)^{\mathfrak{s}-1}|\tilde{y}(\xi)| d \xi+\sum_{j=1}^{n} \frac{1}{\Gamma(\mathfrak{s})} \int_{t_{j-1}}^{t_{j}}\left(t_{j}-\xi\right)^{\mathfrak{s}-1}|\tilde{y}(\xi)| d \xi
\end{aligned}
$$


By $\left[H_{6}\right]$, for $t \in \mathcal{J}_{j}, j=1,2, \ldots, n$, we have

$$
\begin{aligned}
|\tilde{y}(t)| & \leq\left|\mathcal{F}^{\prime}(t, x(t), \tilde{y}(t))\right|+\int_{0}^{t} \frac{(t-\xi)^{\sigma-1}}{\Gamma(\delta)}\left|\mathcal{G}^{\prime}(\xi, x(\xi), \tilde{y}(\xi))\right| d \xi \\
& \leq l_{1}(t)+m_{1}(t)|x(t)|+n_{1}(t)|\tilde{y}(t)|+\frac{t^{\sigma}}{\sigma \Gamma(\delta)}\left(l_{2}(t)+m_{2}(t)|x(t)|+n_{2}(t)|\tilde{y}(t)|\right) \\
& \leq l_{1}^{\star}+m_{1}^{*}\|x\|_{y}+n_{1}^{*}\|\tilde{y}\|_{y}+\frac{\mathrm{T}^{\sigma}}{\sigma \Gamma(\delta)}\left(l_{2}^{\star}+m_{2}^{*}\|x\|_{y}+n_{2}^{*}\|\tilde{y}\|_{y}\right) .
\end{aligned}
$$

Therefore, we get

$$
|\tilde{y}(t)| \leq\|\tilde{y}\|_{y} \leq \frac{l_{1}^{\star}+m_{1}^{\star}\|x\|_{y}}{1-n_{1}^{\star}-n_{2}^{\star} \frac{T^{\sigma}}{\sigma \Gamma(\delta)}}+\frac{\mathrm{T}^{\sigma}}{\sigma \Gamma(\delta)} \frac{l_{2}^{\star}+m_{2}^{\star}\|x\|_{y}}{1-n_{1}^{\star}-n_{2}^{\star} \frac{\mathrm{T}^{\sigma}}{\sigma \Gamma(\delta)}}=\hbar .
$$

Using (3.35) in (3.34) and after simplification, we get

$$
\left\|\mathbb{F}_{\mathfrak{s}} y\right\|_{y} \leq \eta_{2} \text {. }
$$

Hence

$$
\left\|\mathbb{F}_{\mathfrak{s}}(x, y)\right\|_{y} \leq \eta_{2}
$$

Thus

$$
\|\mathbb{F}(x, y)\|\left\|_{x \times y} \leq\right\| \mathbb{F}_{\mathfrak{r}}(x, y)+\mathbb{F}_{\mathfrak{s}}(x, y) \|_{x \times y} \leq \eta_{1}+\eta_{2}=\mathbf{R}_{1} .
$$

Which implies that $\mathbb{F}$ is uniformly bounded on $\mathcal{B}$.

Take a bounded subset $\mathbb{C}$ of $\mathcal{B}$ and $(x, y) \in \mathbb{C}$. Then for $t_{1}, t_{2} \in \mathcal{J}_{i}, i=1,2, \ldots, m$ with $0 \leq t_{1} \leq t_{2} \leq 1$, we have

$$
\left|\mathbb{F}_{\mathfrak{r}} x\left(t_{1}\right)-\mathbb{F}_{\mathfrak{r}} x\left(t_{2}\right)\right| \leq \frac{1}{\Gamma(\mathfrak{r})} \int_{t_{i}}^{t_{2}}\left[\left(t_{2}-\xi\right)^{\mathfrak{r}-1}-\left(t_{1}-\xi\right)^{\mathfrak{r}-1}\right]|\tilde{x}(\xi)| d \xi+\frac{1}{\Gamma(\mathfrak{r})} \int_{t_{2}}^{t_{1}}\left(t_{1}-\xi\right)^{\mathfrak{r}-1}|\tilde{x}(\xi)| d \xi .
$$

By $\left[H_{5}\right]$, for $t \in \mathcal{J}_{i}, i=1,2, \ldots, m$, we have

$$
\begin{aligned}
|\tilde{x}(t)| & \leq|\mathcal{F}(t, x(t), \tilde{x}(t))|+\int_{0}^{t} \frac{(t-\xi)^{\sigma-1}}{\Gamma(\delta)}|\mathcal{G}(\xi, x(\xi), \tilde{x}(\xi))| d \xi \\
& \leq a_{1}(t)+b_{1}(t)|x(t)|+c_{1}(t)|\tilde{x}(t)|+\frac{t^{\sigma}}{\sigma \Gamma(\delta)}\left(a_{2}(t)+b_{2}(t)|x(t)|+c_{2}(t)|\tilde{x}(t)|\right) \\
& \leq a_{1}^{\star}+b_{1}^{\star}\|x\|_{x}+c_{1}^{\star}\|\tilde{x}\|_{x}+\frac{\mathrm{T}^{\sigma}}{\sigma \Gamma(\delta)}\left(a_{2}^{\star}+b_{2}^{\star}\|x\|_{x}+c_{2}^{\star}\|\tilde{x}\|_{x}\right) .
\end{aligned}
$$

Therefore, we get

$$
|\tilde{x}(t)| \leq\|\tilde{x}\|_{x} \leq \frac{a_{1}^{\star}+b_{1}^{\star}\|x\|_{x}}{1-c_{1}^{\star}-c_{2}^{\star} \frac{T^{\sigma}}{\sigma \Gamma(\delta)}}+\frac{\mathrm{T}^{\sigma}}{\sigma \Gamma(\delta)} \frac{a_{2}^{\star}+b_{2}^{\star}\|x\|_{x}}{1-c_{1}^{\star}-c_{2}^{\star} \frac{\mathrm{T}^{\sigma}}{\sigma \Gamma(\delta)}}=\hbar .
$$

Using (3.37) in (3.36) we see that the right-hand side of (3.36) tends to zero as $t_{1} \rightarrow t_{2}$.

Thus

$$
\left|\mathbb{F}_{\mathfrak{r}} x\left(t_{1}\right)-\mathbb{F}_{\mathfrak{r}} x\left(t_{2}\right)\right| \rightarrow 0 \text { as } t_{1} \rightarrow t_{2}
$$

Similarly,

$$
\left|\mathbb{F}_{\mathfrak{r}} y\left(t_{1}\right)-\mathbb{F}_{\mathfrak{r}} y\left(t_{2}\right)\right| \rightarrow 0 \text { as } t_{1} \rightarrow t_{2} .
$$

Now for any $t_{1}, t_{2} \in \mathcal{J}_{j}, j=1,2, \ldots, n$ with $0 \leq t_{1} \leq t_{2} \leq 1$, we have

$$
\left|\mathbb{F}_{\mathfrak{s}} x\left(t_{1}\right)-\mathbb{F}_{\mathfrak{s}} x\left(t_{2}\right)\right| \leq \frac{1}{\Gamma(\mathfrak{s})} \int_{t_{j}}^{t_{2}}\left[\left(t_{2}-\xi\right)^{\mathfrak{s}-1}-\left(t_{1}-\xi\right)^{\mathfrak{s}-1}\right]|\tilde{y}(\xi)| d \xi+\frac{1}{\Gamma(\mathfrak{s})} \int_{t_{2}}^{t_{1}}\left(t_{1}-\xi\right)^{\mathfrak{s}-1}|\tilde{y}(\xi)| d \xi .
$$


By $\left[H_{6}\right]$, for $t \in \mathcal{J}_{j}, j=1,2, \ldots, n$, we have

$$
\begin{aligned}
|\tilde{y}(t)| & \leq\left|\mathcal{F}^{\prime}(t, x(t), \tilde{y}(t))\right|+\int_{0}^{t} \frac{(t-\xi)^{\sigma-1}}{\Gamma(\delta)}\left|\mathcal{G}^{\prime}(\xi, x(\xi), \tilde{y}(\xi))\right| d \xi \\
& \leq l_{1}(t)+m_{1}(t)|x(t)|+n_{1}(t)|\tilde{y}(t)|+\frac{t^{\sigma}}{\sigma \Gamma(\delta)}\left(l_{2}(t)+m_{2}(t)|x(t)|+n_{2}(t)|\tilde{y}(t)|\right) \\
& \leq l_{1}^{\star}+m_{1}^{\star}\|x\|_{y}+n_{1}^{\star}\|\tilde{y}\|_{y}+\frac{\mathrm{T}^{\sigma}}{\sigma \Gamma(\delta)}\left(l_{2}^{\star}+m_{2}^{\star}\|x\|_{y}+n_{2}^{\star}\|\tilde{y}\|_{y}\right) .
\end{aligned}
$$

Therefore, we get

$$
|\tilde{y}(t)| \leq\|\tilde{y}\|_{y} \leq \frac{l_{1}^{\star}+m_{1}^{\star}\|x\|_{y}}{1-n_{1}^{\star}-n_{2}^{\star} \frac{\mathrm{T}^{\sigma}}{\sigma \Gamma(\delta)}}+\frac{\mathrm{T}^{\sigma}}{\sigma \Gamma(\delta)} \frac{l_{2}^{\star}+m_{2}^{\star}\|x\| y}{1-n_{1}^{\star}-n_{2}^{\star} \frac{\mathrm{T}^{\sigma}}{\sigma \Gamma(\delta)}}=\hbar .
$$

Using (3.39) in (3.38), we see that the right-hand side of (3.38) tends to zero as $t_{1} \rightarrow t_{2}$.

Similarly,

$$
\left|\mathbb{F}_{\mathfrak{s}} y\left(t_{1}\right)-\mathbb{F}_{\mathfrak{s}} y\left(t_{2}\right)\right| \rightarrow 0 \text { as } t_{1} \rightarrow t_{2}
$$

Thus

$$
\left|\mathbb{F}(x, y)\left(t_{1}\right)-\mathbb{F}(x, y)\left(t_{2}\right)\right| \rightarrow 0 \text { as } t_{1} \rightarrow t_{2} .
$$

Hence, $\mathbb{F}$ is equicontinuous and by the Arzela-Ascoli theorem, $\mathbb{F}$ is compact. By Theorem 2.7, system (1.2) has at least one solution.

Theorem 3.8. If $\Delta=\max \left(\Delta_{1}, \Delta_{2}\right)<1$, then under the hypothesis $\left[H_{1}\right]-\left[H_{9}\right]$, system (1.2) has a unique solution.

Proof. Suppose $x, \bar{x} \in X$ and for $t \in \mathcal{J}_{i}, i=1,2, \ldots, m$, we have

$$
\begin{aligned}
\left|\mathcal{T}_{\mathfrak{r}} x(t)-\mathcal{T}_{\mathfrak{r}} \bar{x}(t)\right| \leq & \frac{1}{\Gamma(\mathfrak{r})} \int_{t_{i}}^{t}(t-\xi)^{\mathfrak{r}-1}|\tilde{x}(\xi)-\tilde{x}(\xi)| d \xi+\sum_{i=1}^{m} \frac{1}{\Gamma(\mathfrak{r})} \int_{t_{i-1}}^{t_{i}}\left(t_{i}-\xi\right)^{\mathfrak{r}-1}|\tilde{x}(\xi)-\tilde{x}(\xi)| d \xi \\
& +\sum_{i=1}^{m}\left|I_{i}\left(x\left(t_{i}\right)\right)-I_{i}\left(\bar{x}\left(t_{i}\right)\right)\right|+|\mathrm{h}(x)-\mathrm{h}(\bar{x})|,
\end{aligned}
$$

where

$$
\tilde{x}(t)=\mathcal{F}(t, y(t), \tilde{x}(t))+\int_{0}^{t} \frac{(t-\xi)^{\sigma-1}}{\Gamma(\delta)} \mathcal{G}(\xi, y(\xi), \tilde{x}(\xi)) d \xi
$$

and

$$
\overline{\tilde{x}}(t)=\mathcal{F}(t, \bar{y}(t), \overline{\tilde{x}}(t))+\int_{0}^{t} \frac{(t-\xi)^{\sigma-1}}{\Gamma(\delta)} \mathcal{G}(\xi, \bar{y}(\xi), \overline{\tilde{x}}(\xi)) d \xi
$$

Using $\left[H_{1}\right]$, we have

$$
\begin{aligned}
|\tilde{x}(t)-\overline{\tilde{x}}(t)| & =\left|\mathcal{F}(t, y(t), \tilde{x}(t))+\int_{0}^{t} \frac{(t-\xi)^{\sigma-1}}{\Gamma(\delta)} \mathcal{G}(\xi, y(\xi), \tilde{x}(\xi)) d \xi-\mathcal{F}(t, \bar{y}(t), \overline{\tilde{x}}(t))-\int_{0}^{t} \frac{(t-\xi)^{\sigma-1}}{\Gamma(\delta)} \mathcal{G}(\xi, \bar{y}(\xi), \overline{\tilde{x}}(\xi)) d \xi\right| \\
& \leq|\mathcal{F}(t, y(t), \tilde{x}(t))-\mathcal{F}(t, \bar{y}(t), \overline{\tilde{x}}(t))|+\int_{0}^{t} \frac{(t-\xi)^{\sigma-1}}{\Gamma(\delta)}|\mathcal{G}(\xi, y(\xi), \tilde{x}(\xi))-\mathcal{G}(\xi, \bar{y}(\xi), \overline{\tilde{x}}(\xi))| d \xi \\
& \leq \mathrm{M}_{1}|y(t)-\bar{y}(t)|+\mathrm{N}_{1}|\tilde{x}(t)-\overline{\tilde{x}}(t)|+\frac{t^{\sigma}}{\sigma \Gamma(\delta)}\left(\mathrm{M}_{2}|y(t)-\bar{y}(t)|+\mathrm{N}_{2}|\tilde{x}(t)-\overline{\tilde{x}}(t)|\right) .
\end{aligned}
$$


Thus

$$
|\tilde{x}(t)-\overline{\tilde{x}}(t)| \leq\left(\frac{\mathrm{M}_{1}}{1-\mathrm{N}_{1}-\mathrm{N}_{2} \frac{t^{\sigma}}{\sigma \Gamma(\delta)}}+\frac{\mathrm{M}_{2} \frac{t^{\sigma}}{\sigma \Gamma(\delta)}}{1-\mathrm{N}_{1}-\mathrm{N}_{2} \frac{t^{\sigma}}{\sigma \Gamma(\delta)}}\right)|y(t)-\bar{y}(t)| .
$$

Using hypothesis $\left[H_{1}\right],\left[H_{3}\right]$ and $\left[H_{4}\right]$, inequality (3.40) implies

$$
\begin{aligned}
\left|\mathcal{T}_{\mathfrak{r}} \chi(t)-\mathcal{T}_{\mathfrak{r}} \bar{x}(t)\right| \leq & {\left[\left(\frac{(1+m) t^{\mathfrak{r}}}{\Gamma(\mathfrak{r}+1)}\right)\left(\frac{\mathrm{M}_{1}}{1-\mathrm{N}_{1}-\mathrm{N}_{2} \frac{t^{\sigma}}{\sigma \Gamma(\delta)}}+\frac{\mathrm{M}_{2} \frac{t^{\sigma}}{\sigma \Gamma(\delta)}}{1-\mathrm{N}_{1}-\mathrm{N}_{2} \frac{t^{\sigma}}{\sigma \Gamma(\delta)}}\right)\right]|y(t)-\bar{y}(t)| } \\
& +\left(m A_{I_{i}}+A_{\mathrm{h}}\right)|x(t)-\bar{x}(t)| .
\end{aligned}
$$

Taking norm on both sides, we have

$$
\begin{aligned}
\left\|\mathcal{T}_{r}(x)-\mathcal{T}_{r}(\bar{x})\right\|_{X} \leq & {\left[\left(\frac{(1+m) \mathrm{T}^{\mathfrak{r}}}{\Gamma(\mathfrak{r}+1)}\right)\left(\frac{\mathrm{M}_{1}}{1-\mathrm{N}_{1}-\mathrm{N}_{2} \frac{\mathrm{T}^{\sigma}}{\sigma \Gamma(\delta)}}+\frac{\mathrm{M}_{2} \frac{\mathrm{T}^{\sigma}}{\sigma \Gamma(\delta)}}{1-\mathrm{N}_{1}-\mathrm{N}_{2} \frac{\mathrm{T}^{\sigma}}{\sigma \Gamma(\delta)}}\right)\right]\|y-\bar{y}\|_{x} } \\
& +\left(m A_{I_{i}}+A_{\mathrm{h}}\right)\|x-\bar{x}\|_{x} .
\end{aligned}
$$

Similarly, for $x, \bar{x} \in \mathcal{X}$ and $t \in \mathcal{J}_{0}$, we get

$$
\left\|\mathcal{T}_{\mathfrak{r}}(x)-\mathcal{T}_{\mathfrak{r}}(\bar{x})\right\|_{x} \leq\left[\frac{\mathrm{T}^{\mathfrak{r}}}{\Gamma(\mathfrak{r}+1)}\left(\frac{\mathrm{M}_{1}}{1-\mathrm{N}_{1}-\mathrm{N}_{2} \frac{\mathrm{T}^{\sigma}}{\sigma \Gamma(\delta)}}+\frac{\mathrm{M}_{2} \frac{\mathrm{T}^{\sigma}}{\sigma \Gamma(\delta)}}{1-\mathrm{N}_{1}-\mathrm{N}_{2} \frac{\mathrm{T}^{\sigma}}{\sigma \Gamma(\delta)}}\right)\right]\|y-\bar{y}\|_{x}+A_{\mathrm{h}}\|x-\bar{x}\|_{X} .
$$

In the same manner, we can obtain

$$
\left\|\mathcal{T}_{\mathfrak{r}}(y)-\mathcal{T}_{\mathfrak{r}}(\bar{y})\right\|_{x} \leq\left[\left(\frac{(1+m) \mathrm{T}^{\mathfrak{r}}}{\Gamma(\mathfrak{r}+1)}\right)\left(\frac{\mathrm{M}_{1}}{1-\mathrm{N}_{1}-\mathrm{N}_{2} \frac{\mathrm{T}^{\sigma}}{\sigma \Gamma(\delta)}}+\frac{\mathrm{M}_{2} \frac{\mathrm{T}^{\sigma}}{\sigma \Gamma(\delta)}}{1-\mathrm{N}_{1}-\mathrm{N}_{2} \frac{\mathrm{T}^{\sigma}}{\sigma \Gamma(\delta)}}\right)\right]\|y-\bar{y}\|_{x}+\left(m A_{I_{i}}+A_{\mathrm{h}}\right)\|x-\bar{x}\|_{x}
$$

Similarly, for $y, \bar{y} \in \mathcal{X}$ and $t \in \mathcal{J}_{0}$, we get

$$
\left\|\mathcal{T}_{\mathfrak{r}}(y)-\mathcal{T}_{\mathfrak{r}}(\bar{y})\right\|_{x} \leq\left[\frac{\mathrm{T}^{\mathfrak{r}}}{\Gamma(\mathfrak{r}+1)}\left(\frac{\mathrm{M}_{1}}{1-\mathrm{N}_{1}-\mathrm{N}_{2} \frac{\mathrm{T}^{\sigma}}{\sigma \Gamma(\delta)}}+\frac{\mathrm{M}_{2} \frac{\mathrm{T}^{\sigma}}{\sigma \Gamma(\delta)}}{1-\mathrm{N}_{1}-\mathrm{N}_{2} \frac{\mathrm{T}^{\sigma}}{\sigma \Gamma(\delta)}}\right)\right]\|y-\bar{y}\|_{x}+A_{\mathrm{h}}\|x-\bar{x}\|_{x} .
$$

So from (3.41) and (3.42), we get

$$
\left\|\mathcal{T}_{\mathfrak{r}}(x, y)-\mathcal{T}_{\mathfrak{r}}(\bar{x}, \bar{y})\right\|_{x} \leq \Delta_{1}\|(x, y)-(\bar{x}, \bar{y})\|_{X}
$$

Now, suppose $x, \bar{x} \in X$ and for $t \in \mathcal{J}_{j}, j=1,2, \ldots, n$, we have

$$
\begin{aligned}
\left|\mathcal{T}_{\mathfrak{s}} x(t)-\mathcal{T}_{\mathfrak{s}} \bar{x}(t)\right| \leq & \frac{1}{\Gamma(\mathfrak{s})} \int_{t_{j}}^{t}(t-\xi)^{\mathfrak{s}-1}|\tilde{y}(\xi)-\overline{\tilde{y}}(\xi)| d \xi+\sum_{j=1}^{n} \frac{1}{\Gamma(\mathfrak{s})} \int_{t_{j-1}}^{t_{j}}\left(t_{j}-\xi\right)^{\mathfrak{s}-1}|\tilde{y}(\xi)-\bar{y}(\xi)| d \xi \\
& +\sum_{j=1}^{n}\left|I_{j}\left(y\left(t_{j}\right)\right)-I_{j}\left(\bar{y}\left(t_{j}\right)\right)\right|+|\mathrm{g}(y)-\mathrm{g}(\bar{y})|
\end{aligned}
$$

where

$$
\tilde{y}(t)=\mathcal{F}^{\prime}(t, x(t), \tilde{y}(t))+\int_{0}^{t} \frac{(t-\xi)^{\sigma-1}}{\Gamma(\delta)} \mathcal{G}^{\prime}(\xi, x(\xi), \tilde{y}(\xi)) d \xi
$$

and

$$
\overline{\tilde{y}}(t)=\mathcal{F}^{\prime}(t, \bar{x}(t), \overline{\tilde{y}}(t))+\int_{0}^{t} \frac{(t-\xi)^{\sigma-1}}{\Gamma(\delta)} \mathcal{G}^{\prime}(\xi, \bar{x}(\xi), \overline{\tilde{y}}(\xi)) d \xi
$$


Using $\left[H_{2}\right]$, we have

$$
\begin{aligned}
|\tilde{y}(t)-\overline{\tilde{y}}(t)| & =\left|\mathcal{F}^{\prime}(t, x(t), \tilde{y}(t))+\int_{0}^{t} \frac{(t-\xi)^{\sigma-1}}{\Gamma(\delta)} \mathcal{G}^{\prime}(\xi, x(\xi), \tilde{y}(\xi)) d \xi-\mathcal{F}^{\prime}(t, \bar{x}(t), \overline{\tilde{y}}(t))-\int_{0}^{t} \frac{(t-\xi)^{\sigma-1}}{\Gamma(\delta)} \mathcal{G}^{\prime}(\xi, \bar{x}(\xi), \overline{\tilde{y}}(\xi)) d \xi\right| \\
& \leq\left|\mathcal{F}^{\prime}(t, x(t), \tilde{y}(t))-\mathcal{F}^{\prime}(t, \bar{x}(t), \overline{\tilde{y}}(t))\right|+\int_{0}^{t} \frac{(t-\xi)^{\sigma-1}}{\Gamma(\delta)}\left|\mathcal{G}^{\prime}(\xi, x(\xi), \tilde{y}(\xi))-\mathcal{G}^{\prime}(\xi, \bar{x}(\xi), \bar{y}(\xi))\right| d \xi \\
& \leq \mathrm{M}_{1}^{\prime}|x(t)-\bar{x}(t)|+\mathrm{N}_{1}^{\prime}|\tilde{y}(t)-\overline{\tilde{y}}(t)|+\frac{t^{\sigma}}{\sigma \Gamma(\delta)}\left(\mathrm{M}_{2}^{\prime}|x(t)-\bar{x}(t)|+\mathrm{N}_{2}^{\prime}|\tilde{y}(t)-\overline{\tilde{y}}(t)|\right) .
\end{aligned}
$$

Thus

$$
|\tilde{y}(t)-\bar{y}(t)| \leq\left(\frac{\mathrm{M}_{1}^{\prime}}{1-\mathrm{N}_{1}^{\prime}-\mathrm{N}_{2}^{\prime} \frac{t^{\sigma}}{\sigma \Gamma(\delta)}}+\frac{\mathrm{M}_{2}^{\prime} \frac{t^{\sigma}}{\sigma \Gamma(\delta)}}{1-\mathrm{N}_{1}^{\prime}-\mathrm{N}_{2}^{\prime} \frac{t^{\sigma}}{\sigma \Gamma(\delta)}}\right)|x(t)-\bar{x}(t)| .
$$

By using hypothesis $\left[H_{2}\right],\left[H_{3}\right]$ and $\left[H_{4}\right]$, inequality (3.44) implies

$$
\begin{aligned}
\left|\mathcal{T}_{\mathfrak{s}} x(t)-\mathcal{T}_{\mathfrak{s}} \bar{x}(t)\right| \leq & {\left[\left(\frac{(1+n) t^{\mathfrak{s}}}{\Gamma(\mathfrak{s}+1)}\right)\left(\frac{\mathrm{M}_{1}^{\prime}}{1-\mathrm{N}_{1}^{\prime}-\mathrm{N}_{2}^{\prime} \frac{t^{\sigma}}{\sigma \Gamma(\delta)}}+\frac{\mathrm{M}_{2}^{\prime} \frac{t^{\sigma}}{\sigma \Gamma(\delta)}}{1-\mathrm{N}_{1}^{\prime}-\mathrm{N}_{2}^{\prime} \frac{t^{\sigma}}{\sigma \Gamma(\delta)}}\right)\right]|x(t)-\bar{x}(t)| } \\
& +\left(n A_{I_{j}}+A_{\mathrm{g}}\right)|y(t)-\bar{y}(t)| .
\end{aligned}
$$

Taking norm on both sides, we have

$$
\left\|\mathcal{T}_{\mathfrak{s}}(x)-\mathcal{T}_{\mathfrak{s}}(\bar{x})\right\| y \leq\left[\left(\frac{(1+n) \mathrm{T}^{\mathfrak{s}}}{\Gamma(\mathfrak{s}+1)}\right)\left(\frac{\mathrm{M}_{1}^{\prime}}{1-\mathrm{N}_{1}^{\prime}-\mathrm{N}_{2}^{\prime} \frac{\mathrm{T}^{\sigma}}{\sigma \Gamma(\delta)}}+\frac{\mathrm{M}_{2}^{\prime} \frac{\mathrm{T}^{\sigma}}{\sigma \Gamma(\delta)}}{1-\mathrm{N}_{1}^{\prime}-\mathrm{N}_{2}^{\prime} \frac{\mathrm{T}^{\sigma}}{\sigma \Gamma(\delta)}}\right)\right]\|x-\bar{x}\|_{y}+\left(n A_{I_{j}}+A_{\mathfrak{g}}\right)\|y-\bar{y}\|_{y} .
$$

Similarly, for $x, \bar{x} \in \mathcal{y}$ and $t \in \mathcal{J}_{0}$, we get

$$
\left\|\mathcal{T}_{\mathfrak{s}}(x)-\mathcal{T}_{\mathfrak{s}}(\bar{x})\right\|_{y} \leq\left[\frac{\mathrm{T}^{\mathfrak{s}}}{\Gamma(\mathfrak{s}+1)}\left(\frac{\mathrm{M}_{1}^{\prime}}{1-\mathrm{N}_{1}^{\prime}-\mathrm{N}_{2}^{\prime} \frac{\mathrm{T}^{\sigma}}{\sigma \Gamma(\delta)}}+\frac{\mathrm{M}_{2}^{\prime} \frac{\mathrm{T}^{\sigma}}{\sigma \Gamma(\delta)}}{1-\mathrm{N}_{1}^{\prime}-\mathrm{N}_{2}^{\prime} \frac{\mathrm{T}^{\sigma}}{\sigma \Gamma(\delta)}}\right)\right]\|x-\bar{x}\|_{y}+A_{\mathfrak{g}}\|y-\bar{y}\|_{y} .
$$

In the same manner, we can obtain

$$
\begin{aligned}
\left\|\mathcal{T}_{\mathfrak{s}}(y)-\mathcal{T}_{\mathfrak{s}}(\bar{y})\right\|_{y} \leq & {\left[\left(\frac{(1+n) \mathrm{T}^{\mathfrak{s}}}{\Gamma(\mathfrak{s}+1)}\right)\left(\frac{\mathrm{M}_{1}^{\prime}}{1-\mathrm{N}_{1}^{\prime}-\mathrm{N}_{2}^{\prime} \frac{\mathrm{T}^{\sigma}}{\sigma \Gamma(\delta)}}+\frac{\mathrm{M}_{2}^{\prime} \frac{\mathrm{T}^{\sigma}}{\sigma \Gamma(\delta)}}{1-\mathrm{N}_{1}^{\prime}-\mathrm{N}_{2}^{\prime} \frac{\mathrm{T}^{\sigma}}{\sigma \Gamma(\delta)}}\right)\right]\|y-\bar{y}\|_{y} } \\
& +\left(n A_{I_{j}}+A_{\mathfrak{g}}\right)\|x-\bar{x}\|_{y} .
\end{aligned}
$$

Similarly, for $y, \bar{y} \in y$ and $t \in \mathcal{J}_{0}$, we get

$$
\begin{aligned}
& \left\|\mathcal{T}_{\mathfrak{s}}(y)-\mathcal{T}_{\mathfrak{s}}(\bar{y})\right\|_{\mathfrak{y}} \leq\left[\frac{\mathrm{T}^{\mathfrak{s}}}{\Gamma(\mathfrak{s}+1)}\left(\frac{\mathrm{M}_{1}^{\prime}}{1-\mathrm{N}_{1}^{\prime}-\mathrm{N}_{2}^{\prime} \frac{\mathrm{T}^{\sigma}}{\sigma \Gamma(\delta)}}+\frac{\mathrm{M}_{2}^{\prime} \frac{\mathrm{T}^{\sigma}}{\sigma \Gamma(\delta)}}{1-\mathrm{N}_{1}^{\prime}-\mathrm{N}_{2}^{\prime} \frac{\mathrm{T}^{\sigma}}{\sigma \Gamma(\delta)}}\right)\right]\|y-\bar{y}\|_{y} \\
& +A \mathrm{~g}\|x-\bar{x}\| y .
\end{aligned}
$$

So from (3.45) and (3.46), we get

$$
\left\|\mathcal{T}_{\mathfrak{s}}(x, y)-\mathcal{T}_{\mathfrak{s}}(\bar{x}, \bar{y})\right\|_{y} \leq \Delta_{2}\|(x, y)-(\bar{x}, \bar{y})\|_{y} .
$$

Hence, it follows that

$$
\|\mathcal{T}(x, y)-\mathcal{T}(\bar{x}, \bar{y})\|_{x \times y} \leq \max \left(\Delta_{1}, \Delta_{2}\right)\left(\|x-\bar{x}\|_{x \times y}+\|y-\bar{y}\|_{x \times y}\right) .
$$

Which implies that $\mathcal{T}$ is contraction, hence it has a unique fixed point. 


\section{Ulam stability results}

In this section, we investigate HU stability and its various kinds for problem (1.1). The following definitions are adopted from [7].

For $x \in \mathcal{M}, \epsilon_{\mathfrak{r}}>0, \phi_{\mathfrak{r}} \leq 0$ and a nondecreasing function $\psi_{\mathfrak{r}} \in C\left(\mathcal{J}, \mathbb{R}_{+}\right)$, the following set of inequalities satisfy:

$$
\begin{aligned}
& \left\{\begin{array}{l}
\mid{ }^{c} D^{\mathfrak{r}} x(t)-\mathcal{F}\left(t, x(t),{ }^{c} D^{\mathfrak{r}} x(t)\right)-\int_{0}^{t} \frac{(t-\xi)}{\Gamma(\delta)} \mathcal{G - 1} \\
\left|\Delta x\left(t_{i}\right)-I_{i}\left(x\left(t_{i}\right)\right)\right| \leq \epsilon_{\mathfrak{r}}, i=1,2, \ldots, m,
\end{array}\right. \\
& \left\{\begin{array}{l}
\left|{ }^{c} D^{\mathfrak{r}} x(t)-\mathcal{F}\left(t, x(t),{ }^{c} D^{\mathfrak{r}} x(\xi)\right) d \xi\right| \leq \epsilon_{\mathfrak{r}}, t \in \mathcal{J}_{i}, i=1,2, \ldots, m, \\
\left|\Delta x\left(t_{i}\right)-I_{i}\left(x\left(t_{i}\right)\right)\right| \leq \phi_{\mathfrak{r}}, i=1,2, \ldots, m
\end{array}\right.
\end{aligned}
$$

and

$$
\left\{\begin{array}{l}
\left|{ }^{c} D^{\mathfrak{r}} x(t)-\mathcal{F}\left(t, x(t),{ }^{c} D^{\mathfrak{r}} x(t)\right)-\int_{0}^{t} \frac{(t-\xi)^{\sigma-1}}{\Gamma(\delta)} \mathcal{G}\left(\xi, x(\xi),{ }^{c} D^{\mathfrak{r}} x(\xi)\right) d \xi\right| \leq \epsilon_{\mathfrak{r}} \psi_{\mathfrak{r}}(t), t \in \mathcal{J}_{i}, i=1,2, \ldots, m, \\
\left|\Delta x\left(t_{i}\right)-I_{i}\left(x\left(t_{i}\right)\right)\right| \leq \epsilon_{\mathfrak{r}} \phi_{\mathfrak{r}}, i=1,2, \ldots, m .
\end{array}\right.
$$

Definition 4.1. The problem (1.1) is said to be HU stable if $\exists$ a real number $\mathcal{C}_{\mathcal{F}, \mathcal{G}}>0$, so that for each $\epsilon_{\mathfrak{r}}>0$ and any solution $x \in \mathcal{M}$ of the inequality (4.1), $\exists$ a unique solution $x^{\star} \in \mathcal{M}$ of problem (1.1), so that

$$
\left|x(t)-x^{\star}(t)\right| \leq \mathcal{C}_{\mathcal{F}, \mathcal{G}} \epsilon_{\mathfrak{r}}, \forall t \in \mathcal{J} .
$$

Definition 4.2. The problem (1.1) is said to be generalized HU stable if $\exists$ a function $\vartheta \in \mathcal{C}\left(\mathbb{R}_{+}, \mathbb{R}_{+}\right)$, with $\vartheta(0)=0$, so that for each $\epsilon_{\mathfrak{r}}>0$ and any solution $x \in \mathcal{M}$ of the inequality (4.1), $\exists$ a unique solution $x^{\star} \in \mathcal{M}$ of problem (1.1), so that

$$
\left|x(t)-x^{\star}(t)\right| \leq \mathcal{C}_{\mathcal{F}, \mathcal{G}} \vartheta\left(\epsilon_{\mathfrak{r}}\right), \forall t \in \mathcal{J} .
$$

Definition 4.3. The problem (1.1) is said to be HU-Rassias stable with respect to $\left(\phi_{\mathfrak{r}}, \psi_{\mathfrak{r}}\right)$, if $\exists$ a real number $\mathcal{C}_{\mathcal{F}, \mathcal{G}}>0$, so that for each $\epsilon_{\mathfrak{r}}>0$ and any solution $x \in \mathcal{M}$ of the inequality (4.3), $\exists$ a unique solution $x^{\star} \in \mathcal{M}$ of problem (1.1), so that

$$
\left|x(t)-x^{\star}(t)\right| \leq \mathcal{C}_{\mathcal{F}, \mathcal{G}} \epsilon_{\mathfrak{r}}\left(\phi_{\mathfrak{r}}+\psi_{\mathfrak{r}}(t)\right), \forall t \in \mathcal{J} .
$$

Definition 4.4. The problem (1.1) is said to be generalized HU-Rassias stable with respect to $\left(\phi_{\mathfrak{r}}, \psi_{\mathfrak{r}}\right)$, if $\exists$ a real number $\mathcal{C}_{\mathcal{F}, \mathcal{G}}>0$, so that for each $\epsilon_{\mathfrak{r}}>0$ and any solution $x \in \mathcal{M}$ of the inequality (4.2), $\exists$ a unique solution $x^{\star} \in \mathcal{M}$ of problem (1.1), so that

$$
\left|x(t)-x^{\star}(t)\right| \leq \mathcal{C}_{\mathcal{F}, \mathcal{G}}\left(\phi_{\mathfrak{r}}+\psi_{\mathfrak{r}}(t)\right), \forall t \in \mathcal{J} .
$$

Remark 4.5. Definition 4.1 implies Definition 4.2 and Definition 4.3 implies Definition 4.4.

Remark 4.6. A function $x \in \mathcal{M}$ is a solution of the inequality (4.1) if $\exists$ a function $\Phi \in \mathcal{M}$ and a sequence $\Phi_{i}$ (which depends on $x$ ) so that

i) $|\Phi(t)| \leq \epsilon_{\mathfrak{r}},\left|\Phi_{i}\right| \leq \epsilon_{\mathfrak{r}}, \forall t \in \mathcal{J}, i=1,2, \ldots, m$;

ii) ${ }^{c} D^{\mathfrak{r}} x(t)=\mathcal{F}\left(t, x(t),{ }^{c} D^{\mathfrak{r}} x(t)\right)+\int_{0}^{t} \frac{(t-\xi) \sigma^{\sigma-1}}{\Gamma(\delta)} \mathcal{G}\left(\xi, x(\xi),{ }^{c} D^{\mathfrak{r}} x(\xi)\right) d \xi+\Phi(t), \forall t \in \mathcal{J}$; 
and

iii) $\Delta x\left(t_{i}\right)=I_{i}\left(x\left(t_{i}\right)\right)+\Phi_{i}, \forall t \in \mathcal{J}, i=1,2, \ldots, m$.

Remark 4.7. A function $x \in \mathcal{M}$ is a solution of the inequality (4.2) if $\exists$ a function $\Phi \in \mathcal{M}$ and a sequence $\Phi_{i}$ (which depends on $x$ ) so that

i) $|\Phi(t)| \leq \psi_{\mathfrak{r}}(t),\left|\Phi_{i}\right| \leq \phi_{\mathfrak{r}}, \forall t \in \mathcal{J}, i=1,2, \ldots, m$;

ii) ${ }^{c} D^{\mathfrak{r}} x(t)=\mathcal{F}\left(t, x(t),{ }^{c} D^{\mathfrak{r}} x(t)\right)+\int_{0}^{t} \frac{(t-\xi)^{\sigma-1}}{\Gamma(\delta)} \mathcal{G}\left(\xi, x(\xi),{ }^{c} D^{\mathfrak{r}} x(\xi)\right) d \xi+\Phi(t), \forall t \in \mathcal{J}$;

and

iii) $\Delta x\left(t_{i}\right)=I_{i}\left(x\left(t_{i}\right)\right)+\Phi_{i}, \forall t \in \mathcal{J}, i=1,2, \ldots, m$.

Remark 4.8. A function $x \in \mathcal{N}$ is a solution of the inequality (4.3) if $\exists$ a function $\Phi \in \mathcal{M}$ and a sequence $\Phi_{i}$ (which depends on $x$ ) so that

i) $|\Phi(t)| \leq \epsilon_{\mathfrak{r}} \psi_{\mathfrak{r}}(t),\left|\Phi_{i}\right| \leq \epsilon_{\mathfrak{r}} \phi_{\mathfrak{r}}, \forall t \in \mathcal{J}, i=1,2, \ldots, m$;

ii) ${ }^{c} D^{\mathfrak{r}} x(t)=\mathcal{F}\left(t, x(t),{ }^{c} D^{\mathfrak{r}} x(t)\right)-\int_{0}^{t} \frac{(t-\xi)^{\sigma-1}}{\Gamma(\delta)} \mathcal{G}\left(\xi, x(\xi),{ }^{c} D^{\mathfrak{r}} x(\xi)\right) d \xi+\Phi(t), \forall t \in \mathcal{J}$;

and

iii) $\Delta x\left(t_{i}\right)=I_{i}\left(x\left(t_{i}\right)\right)+\Phi_{i}, \forall t \in \mathcal{J}, i=1,2, \ldots, m$.

Definition 4.9. A function $x \in \mathcal{J}$ is a solution of the problem (1.1) if $x$ satisfies (1.1) with its conditions on $\mathcal{J}$.

Theorem 4.10. If the hypothesis $\left[A_{1}\right]-\left[A_{3}\right]$ and inequality (3.14) are satisfied, then problem (1.1) is HU stable and consequently, it is generalized $\mathrm{HU}$ stable.

Proof. Let $x \in \mathcal{M}$ be any solution of the inequality (4.1) and let $x^{\star} \in \mathcal{M}$ be the solution of the following problem:

$$
\left\{\begin{array}{l}
{ }^{c} D^{\mathfrak{r}} x^{*}(t)=\mathcal{F}\left(t, x^{*}(t),{ }^{c} D^{\mathfrak{r}} x^{*}(t)\right)+\int_{0}^{t} \frac{(t-\xi)^{\sigma-1}}{\Gamma(\delta)} \mathcal{G}\left(\xi, x^{*}(\xi),{ }^{c} D^{\mathfrak{r}} x^{*}(\xi)\right) d \xi, \forall t \in \mathcal{J}, t \neq t_{i} \text { for } i=1,2, \ldots, m, \\
x^{\star}(0)=\mathrm{h}\left(x^{\star}\right), \\
\Delta x^{\star}\left(t_{i}\right)=I_{i}\left(x^{\star}\left(t_{i}\right)\right), i=1,2, \ldots, m .
\end{array}\right.
$$

By Theorem 3.1, for $t \in \mathcal{J}_{i}$, we have

$$
x^{\star}(t)=\frac{1}{\Gamma(\mathfrak{r})} \int_{t_{i}}^{t}(t-\xi)^{\mathfrak{r}-1} \overline{\tilde{\chi}}(\xi) d \xi+\sum_{i=1}^{m}\left[\frac{1}{\Gamma(\mathfrak{r})} \int_{t_{i-1}}^{t_{i}}\left(t_{i}-\xi\right)^{\mathfrak{r}-1} \overline{\tilde{\chi}}(\xi) d \xi+I_{i}\left(x^{\star}\left(t_{i}\right)\right)\right]+\mathrm{h}\left(x^{\star}\right), i=1,2, \ldots, m,
$$

where $\overline{\tilde{x}} \in C(\mathcal{J}, \mathbb{R})$ is given by

$$
\overline{\tilde{x}}(t)=\mathcal{F}(t, \bar{x}(t), \overline{\tilde{x}}(t))+\int_{0}^{t} \frac{(t-\xi)^{\sigma-1}}{\Gamma(\delta)} \mathcal{G}(\xi, \bar{x}(\xi), \bar{x}(\xi)) d \xi .
$$

Since $x$ is a solution of the inequality (4.1), hence by Remark 4.6, we have

$$
\left\{\begin{array}{l}
{ }^{c} D^{\mathfrak{r}} x(t)=\mathcal{F}\left(t, x(t),{ }^{c} D^{\mathfrak{r}} x(t)\right)+\int_{0}^{t} \frac{(t-\xi)^{\sigma-1}}{\Gamma(\delta)} \mathcal{G}\left(\xi, x(\xi),{ }^{c} D^{\mathfrak{r}} x(\xi)\right) d \xi+\Phi(t), \forall t \in \mathcal{J}, t \neq t_{i} \text { for } i=1,2, \ldots, m, \\
x(0)=\mathrm{h}(x), \\
\Delta x\left(t_{i}\right)=I_{i}\left(x\left(t_{i}\right)\right)+\Phi_{i}(t), i=1,2, \ldots, m .
\end{array}\right.
$$


Clearly, the solution of (4.4) will be

$$
x(t)=\left\{\begin{array}{l}
\frac{1}{\Gamma(\mathfrak{r})} \int_{0}^{t}(t-\xi)^{\mathfrak{r}-1} \tilde{x}(\xi) d \xi+\frac{1}{\Gamma(\mathfrak{r})} \int_{0}^{t}(t-\xi)^{\mathfrak{r}-1} \Phi(\xi) d \xi+\mathrm{h}(x), t \in \mathcal{J}_{0}, \\
\frac{1}{\Gamma(\mathfrak{r})} \int_{t_{i}}^{t}(t-\xi)^{\mathfrak{r}-1} \tilde{x}(\xi) d \xi+\frac{1}{\Gamma(\mathfrak{r})} \int_{t_{i}}^{t}(t-\xi)^{\mathfrak{r}-1} \Phi(\xi) d \xi+\sum_{i=1}^{m} \frac{1}{\Gamma(\mathfrak{r})} \int_{t_{i-1}}^{t_{i}}\left(t_{i}-\xi\right)^{\mathfrak{r}-1} \tilde{x}(\xi) d \xi \\
+\sum_{i=1}^{m} \frac{1}{\Gamma(\mathfrak{r})} \int_{t_{i-1}}^{t_{i}}\left(t_{i}-\xi\right)^{\mathfrak{r}-1} \Phi(\xi) d \xi+\sum_{i=1}^{m} I_{i}\left(x\left(t_{i}\right)\right)+\sum_{i=1}^{m} \Phi_{i}+\mathrm{h}(x), t \in \mathcal{J}_{i}, \quad i=1,2, \ldots, m,
\end{array}\right.
$$

where $\tilde{x} \in C(\mathcal{J}, \mathbb{R})$ is given by

$$
\tilde{x}(t)=\mathcal{F}(t, x(t), \tilde{x}(t))+\int_{0}^{t} \frac{(t-\xi)^{\sigma-1}}{\Gamma(\delta)} \mathcal{G}(\xi, x(\xi), \tilde{x}(\xi)) d \xi .
$$

Therefore, for each $t \in \mathcal{J}_{i}, i=1,2, \ldots, m$, we have

$$
\begin{aligned}
\left|x(t)-x^{\star}(t)\right| \leq & \frac{1}{\Gamma(r)} \int_{t_{i}}^{t}(t-\xi)^{r-1}|\tilde{x}(\xi)-\tilde{x}(\xi)| d \xi+\frac{1}{\Gamma(r)} \int_{t_{i}}^{t}(t-\xi)^{r-1}|\Phi(\xi)| d \xi \\
& +\sum_{i=1}^{m} \frac{1}{\Gamma(r)} \int_{t_{i-1}}^{t_{i}}\left(t_{i}-\xi\right)^{r-1}|\tilde{x}(\xi)-\tilde{x}(\xi)| d \xi+\sum_{i=1}^{m} \frac{1}{\Gamma(r)} \int_{t_{i-1}}^{t_{i}}\left(t_{i}-\xi\right)^{r-1}|\Phi(\xi)| d \xi \\
& +\sum_{i=1}^{m}\left|I_{i}\left(x\left(t_{i}\right)\right)-I_{i}\left(x^{\star}\left(t_{i}\right)\right)\right|+\sum_{i=1}^{m}\left|\Phi_{i}\right|+\left|\mathrm{h}(x)-\mathrm{h}\left(x^{\star}\right)\right| .
\end{aligned}
$$

By $\left[A_{2}\right]$, we obtain

$$
|\overline{\tilde{x}}(t)-\tilde{x}(t)| \leq\left(\frac{\mathrm{M}_{1}}{1-\mathrm{N}_{1}-\mathrm{N}_{2} \frac{t^{\sigma}}{\sigma \Gamma(\delta)}}+\frac{\mathrm{M}_{2} \frac{t^{\sigma}}{\sigma \Gamma(\delta)}}{1-\mathrm{N}_{1}-\mathrm{N}_{2} \frac{t^{\sigma}}{\sigma \Gamma(\delta)}}\right)\left|x(t)-x^{\star}(t)\right| .
$$

Hence, using $\left[A_{1}\right]-\left[A_{3}\right]$ and part (i) of Remark 4.6, inequality (4.5) implies that

$$
\begin{aligned}
\left|x(t)-x^{\star}(t)\right| \leq & \frac{t^{\mathfrak{r}}}{\Gamma(\mathfrak{r}+1)}\left(\frac{\mathrm{M}_{1}}{1-\mathrm{N}_{1}-\mathrm{N}_{2} \frac{t^{\sigma}}{\sigma \Gamma(\delta)}}+\frac{\mathrm{M}_{2} \frac{t^{\sigma}}{\sigma \Gamma(\delta)}}{1-\mathrm{N}_{1}-\mathrm{N}_{2} \frac{t^{\sigma}}{\sigma(\delta)}}\right)\left|x(t)-x^{\star}(t)\right|+\frac{\epsilon_{\mathfrak{r}} t^{\mathfrak{r}}}{\Gamma(\mathfrak{r}+1)} \\
& +\frac{m t^{\mathfrak{r}}}{\Gamma(\mathfrak{r}+1)}\left(\frac{\mathrm{M}_{1}}{1-\mathrm{N}_{1}-\mathrm{N}_{2} \frac{t^{\sigma}}{\sigma \Gamma(\delta)}}+\frac{\mathrm{M}_{2} \frac{t^{\sigma}}{\sigma \Gamma(\delta)}}{1-\mathrm{N}_{1}-\mathrm{N}_{2} \frac{t^{\sigma}}{\sigma \Gamma(\delta)}}\right)\left|x(t)-x^{\star}(t)\right|+\frac{m \epsilon_{\mathfrak{r}} t^{\mathfrak{r}}}{\Gamma(\mathfrak{r}+1)} \\
& +m A_{I_{i}}\left|x(t)-x^{\star}(t)\right|+m \epsilon_{\mathfrak{r}}+A_{\mathrm{h}}\left|x(t)-x^{\star}(t)\right| .
\end{aligned}
$$

By taking norm and simplification, we get

$$
\begin{aligned}
\left\|x-x^{*}\right\|_{\mathcal{M}} \leq & \epsilon_{\mathfrak{r}}\left(\frac{(1+m) T^{\mathfrak{r}}}{\Gamma(\mathfrak{r}+1)}+m\right)+\left[\frac{(1+m) \mathrm{T}^{\mathfrak{r}}}{\Gamma(\mathfrak{r}+1)}\left(\frac{\mathrm{M}_{1}}{1-\mathrm{N}_{1}-\mathrm{N}_{2} \frac{\mathrm{T}^{\sigma}}{\sigma \Gamma(\delta)}}+\frac{\mathrm{M}_{2} \frac{\mathrm{T}^{\sigma}}{\sigma \Gamma(\delta)}}{1-\mathrm{N}_{1}-\mathrm{N}_{2} \frac{T^{\sigma}}{\sigma \Gamma(\delta)}}\right)\right. \\
& \left.+A_{\mathrm{h}}+m A_{I_{i}}\right]\left\|x-\chi^{\star}\right\|_{\mathcal{M}} .
\end{aligned}
$$

From which, we obtain

$$
\left\|x-x^{\star}\right\|_{\mathcal{M}} \leq \frac{\epsilon_{\mathfrak{r}}\left(\frac{(1+m) \mathrm{T}^{\mathfrak{r}}}{\Gamma(\mathfrak{r}+1)}+m\right)}{1-\left[\frac{(1+m) \mathrm{T}^{\mathfrak{r}}}{\Gamma(\mathfrak{r}+1)}\left(\frac{\mathrm{M}_{1}}{1-\mathrm{N}_{1}-\mathrm{N}_{2} \frac{\mathrm{T}^{\sigma}}{\sigma \Gamma(\delta)}}+\frac{\mathrm{M}_{2} \frac{T^{\sigma}}{\sigma \Gamma(\delta)}}{1-\mathrm{N}_{1}-\mathrm{N}_{2} \frac{T^{\sigma}}{\sigma \Gamma(\delta)}}\right)+A_{\mathrm{h}}+m A_{I_{i}}\right]} .
$$


Similarly, for $t \in \mathcal{J}_{0}$, we have

$$
\left\|x-x^{\star}\right\|_{\mathcal{M}} \leq \frac{\epsilon_{\mathfrak{r}}\left(\frac{\mathrm{T}^{\mathfrak{r}}}{\Gamma(\mathfrak{r}+1)}\right)}{1-\left[\frac{\mathrm{T}^{\mathfrak{r}}}{\Gamma(\mathfrak{r}+1)}\left(\frac{\mathrm{M}_{1}}{1-\mathrm{N}_{1}-\mathrm{N}_{2} \frac{\mathrm{T}^{\sigma}}{\sigma \Gamma(\delta)}}+\frac{\mathrm{M}_{2} \frac{\mathrm{T}^{\sigma}}{\sigma \Gamma(\delta)}}{1-\mathrm{N}_{1}-\mathrm{N}_{2} \frac{\mathrm{T}^{\sigma}}{\sigma \Gamma(\delta)}}\right)+A_{\mathrm{h}}\right]} .
$$

Combining (4.6) and (4.7), for $t \in \mathcal{J}$, we have

$$
\begin{aligned}
\left\|x-\chi^{*}\right\|_{\mathcal{M}} \leq & {\left[\frac{\left(\frac{(1+m) \mathrm{T}^{\mathfrak{r}}}{\Gamma(\mathfrak{r}+1)}+m\right)}{1-\left[\frac{(1+m) \mathrm{T}^{\mathfrak{r}}}{\Gamma(\mathfrak{r}+1)}\left(\frac{\mathrm{M}_{1}}{1-\mathrm{N}_{1}-\mathrm{N}_{2} \frac{\mathrm{T}^{\sigma}}{\sigma \Gamma(\delta)}}+\frac{\mathrm{M}_{2} \frac{\mathrm{T}^{\sigma}}{\sigma \Gamma(\delta)}}{1-\mathrm{N}_{1}-\mathrm{N}_{2} \frac{\mathrm{T}^{\sigma}}{\sigma \Gamma(\delta)}}\right)+A_{\mathrm{h}}+m A_{I_{i}}\right]}\right.} \\
& \left.+\frac{\left(\frac{\mathrm{T}^{\mathfrak{r}}}{\Gamma(\mathfrak{r}+1)}\right)}{1-\left[\frac{\mathrm{T}^{\mathfrak{r}}}{\Gamma(\mathfrak{r}+1)}\left(\frac{\mathrm{M}_{1}}{1-\mathrm{N}_{1}-\mathrm{N}_{2} \frac{\mathrm{T}^{\sigma}}{\sigma \Gamma(\delta)}}+\frac{\mathrm{M}_{2} \frac{\mathrm{T}^{\sigma}}{\sigma \Gamma(\delta)}}{1-\mathrm{N}_{1}-\mathrm{N}_{2} \frac{\mathrm{T}^{\sigma}}{\sigma \Gamma(\delta)}}\right)+A_{\mathrm{h}}\right]}\right] \epsilon_{\mathfrak{r}} .
\end{aligned}
$$

Thus

$$
\left\|x-\chi^{\star}\right\|_{\mathcal{M}} \leq \mathbf{C}_{1} \epsilon_{\mathfrak{r}},
$$

where

$$
\begin{aligned}
& \mathbf{C}_{1}= \frac{\left(\frac{(1+m) \mathrm{T}^{\mathfrak{r}}}{\Gamma(\mathfrak{r}+1)}+m\right)}{1-\left[\frac{(1+m) \mathrm{T}^{\mathfrak{r}}}{\Gamma(\mathfrak{r}+1)}\left(\frac{\mathrm{M}_{1}}{1-\mathrm{N}_{1}-\mathrm{N}_{2} \frac{\mathrm{T}^{\sigma}}{\sigma \Gamma(\delta)}}+\frac{\mathrm{M}_{2} \frac{\mathrm{T}^{\sigma}}{\sigma \Gamma(\delta)}}{1-\mathrm{N}_{1}-\mathrm{N}_{2} \frac{\mathrm{T}^{\sigma}}{\sigma \Gamma(\delta)}}\right)+A_{\mathrm{h}}+m A_{I_{i}}\right]} \\
&+\frac{\left(\frac{\mathrm{T}^{\mathfrak{r}}}{\Gamma(\mathfrak{r}+1)}\right)}{1-\left[\frac{\mathrm{T}^{\mathfrak{r}}}{\Gamma(\mathfrak{r}+1)}\left(\frac{\mathrm{M}_{1}}{1-\mathrm{N}_{1}-\mathrm{N}_{2} \frac{\mathrm{T}^{\sigma}}{\sigma \Gamma(\delta)}}+\frac{\mathrm{M}_{2} \frac{\mathrm{T}^{\sigma}}{\sigma \Gamma(\delta)}}{1-\mathrm{N}_{1}-\mathrm{N}_{2} \frac{T^{\sigma}}{\sigma \Gamma(\delta)}}\right)+A_{\mathrm{h}}\right]} .
\end{aligned}
$$

Therefore, problem (1.1) is HU stable. Further, if we set $\vartheta\left(\epsilon_{\mathfrak{r}}\right)=C\left(\epsilon_{\mathfrak{r}}\right) ; \vartheta(0)=0$, then the problem (1.1) becomes generalized HU stable.

Assume that

- $\left[A_{7}\right] \exists$ a nondecreasing function $\psi_{\mathfrak{r}} \in \mathcal{M}$ and a constant $\varrho_{\psi_{\mathfrak{r}}}>0$ so that for each $t \in \mathcal{J}:$

$$
I^{\varrho \psi_{\mathrm{r}}(t)} \leq \varrho_{\psi_{\mathrm{r}}} \psi_{\mathrm{r}}(t)
$$

Theorem 4.11. If the hypothesis $\left[A_{1}\right]-\left[A_{3}\right],\left[A_{7}\right]$ and the inequality (3.14) are satisfied, then problem (1.1) is $\mathrm{HU}-$ Rassias stable with respect to $\left(\phi_{\mathfrak{r}}, \psi_{\mathfrak{r}}\right)$ and consequently, it is generalized HU-Rassias stable.

Proof. Let $x \in \mathcal{M}$ be any solution of the inequality (4.3) and let $x^{\star} \in \mathcal{M}$ be the solution of the problem:

$$
\left\{\begin{array}{l}
{ }^{c} D^{\mathfrak{r}} x^{\star}(t)=\mathcal{F}\left(t, x^{\star}(t),{ }^{c} D^{\mathfrak{r}} x^{\star}(t)\right)+\int_{0}^{t} \frac{(t-\xi)^{\sigma-1}}{\Gamma(\delta)} \mathcal{G}\left(\xi, x^{\star}(\xi),{ }^{c} D^{\mathfrak{r}} x^{\star}(\xi)\right) d \xi, \forall t \in \mathcal{J}, t \neq t_{i} \text { for } i=1,2, \ldots, m, \\
x^{\star}(0)=\mathrm{h}\left(x^{\star}\right) \\
\Delta x^{\star}\left(t_{i}\right)=I_{i}\left(x^{\star}\left(t_{i}\right)\right), i=1,2, \ldots, m .
\end{array}\right.
$$

From the proof of Theorem 4.10, we have

$$
\left|x(t)-x^{\star}(t)\right| \leq \frac{1}{\Gamma(\mathfrak{r})} \int_{t_{i}}^{t}(t-\xi)^{\mathfrak{r}-1}|\tilde{\tilde{x}}(\xi)-\tilde{x}(\xi)| d \xi+\frac{1}{\Gamma(\mathfrak{r})} \int_{t_{i}}^{t}(t-\xi)^{\mathfrak{r}-1}|\Phi(\xi)| d \xi
$$




$$
\begin{aligned}
& +\sum_{i=1}^{m} \frac{1}{\Gamma(\mathfrak{r})} \int_{t_{i-1}}^{t_{i}}\left(t_{i}-\xi\right)^{\mathfrak{r}-1}|\overline{\tilde{x}}(\xi)-\tilde{x}(\xi)| d \xi+\sum_{i=1}^{m} \frac{1}{\Gamma(\mathfrak{r})} \int_{t_{i-1}}^{t_{i}}\left(t_{i}-\xi\right)^{\mathfrak{r}-1}|\Phi(\xi)| d \xi \\
& +\sum_{i=1}^{m}\left|I_{i}\left(x\left(t_{i}\right)\right)-I_{i}\left(x^{\star}\left(t_{i}\right)\right)\right|+\sum_{i=1}^{m}\left|\Phi_{i}\right|+\left|\mathrm{h}(x)-\mathrm{h}\left(x^{\star}\right)\right| .
\end{aligned}
$$

By $\left[A_{1}\right]$, we obtain

$$
|\overline{\tilde{x}}(t)-\tilde{x}(t)| \leq\left(\frac{\mathrm{M}_{1}}{1-\mathrm{N}_{1}-\mathrm{N}_{2} \frac{t^{\sigma}}{\sigma \Gamma(\delta)}}+\frac{\mathrm{M}_{2} \frac{t^{\sigma}}{\sigma \Gamma(\delta)}}{1-\mathrm{N}_{1}-\mathrm{N}_{2} \frac{t^{\sigma}}{\sigma \Gamma(\delta)}}\right)\left|x(t)-x^{\star}(t)\right| .
$$

Hence, using $\left[A_{1}\right]-\left[A_{3}\right]$ and part (i) of Remark 4.8, above inequality, we have

$$
\begin{aligned}
\left|x(t)-x^{\star}(t)\right| \leq & \frac{t^{\mathfrak{r}}}{\Gamma(\mathfrak{r}+1)}\left(\frac{\mathrm{M}_{1}}{1-\mathrm{N}_{1}-\mathrm{N}_{2} \frac{t^{\sigma}}{\sigma \Gamma(\delta)}}+\frac{\mathrm{M}_{2} \frac{t^{\sigma}}{\sigma \Gamma(\delta)}}{1-\mathrm{N}_{1}-\mathrm{N}_{2} \frac{t^{\sigma}}{\sigma \Gamma(\delta)}}\right)\left|x(t)-x^{\star}(t)\right|+\frac{\psi_{\mathfrak{r}}(t) \epsilon_{\mathfrak{r}} t^{\mathfrak{r}}}{\Gamma(\mathfrak{r}+1)} \\
& +\frac{m t^{\mathfrak{r}}}{\Gamma(\mathfrak{r}+1)}\left(\frac{\mathrm{M}_{1}}{1-\mathrm{N}_{1}-\mathrm{N}_{2} \frac{t^{\sigma}}{\sigma \Gamma(\delta)}}+\frac{\mathrm{M}_{2} \frac{t^{\sigma}}{\sigma \Gamma(\delta)}}{1-\mathrm{N}_{1}-\mathrm{N}_{2} \frac{t^{\sigma}}{\sigma \Gamma(\delta)}}\right)\left|x(t)-x^{\star}(t)\right|+\frac{m \psi_{\mathfrak{r}}(t) \epsilon_{\mathfrak{r}} t^{\mathfrak{r}}}{\Gamma(\mathfrak{r}+1)} \\
& +m A_{I_{i}}\left|x(t)-x^{\star}(t)\right|+m \epsilon_{\mathfrak{r}}+A_{\mathrm{h}}\left|x(t)-x^{\star}(t)\right| .
\end{aligned}
$$

Using $\left[A_{7}\right]$, we get

$$
\begin{aligned}
\left\|x-x^{\star}\right\|_{\mathcal{M}} \leq & \epsilon_{\mathfrak{r}}\left(\varrho_{\psi_{\mathfrak{r}}} \psi_{\mathfrak{r}}(t)(1+\mathcal{K})+\mathcal{L} \phi_{\mathfrak{r}}\right)+\left[\frac{(1+m) T^{\mathfrak{r}}}{\Gamma(\mathfrak{r}+1)}\left(\frac{\mathrm{M}_{1}}{1-\mathrm{N}_{1}-\mathrm{N}_{2} \frac{\mathrm{T}^{\sigma}}{\sigma \Gamma(\delta)}}+\frac{\mathrm{M}_{2} \frac{\mathrm{T}^{\sigma}}{\sigma \Gamma(\delta)}}{1-\mathrm{N}_{1}-\mathrm{N}_{2} \frac{\mathrm{T}^{\sigma}}{\sigma \Gamma(\delta)}}\right)\right. \\
& \left.+A_{\mathrm{h}}+m A_{I_{i}}\right]\left\|x-x^{\star}\right\|_{\mathcal{M}} \\
\leq & \epsilon_{\mathfrak{r}}\left(\left(\psi_{\mathfrak{r}}(t)+\phi_{\mathfrak{r}}\right)\left(\varrho_{\psi_{\mathfrak{r}}}(1+\mathcal{K})+\mathcal{L} \phi_{\mathfrak{r}}\right)+\left[\frac{(1+m) \mathrm{T}^{\mathfrak{r}}}{\Gamma(\mathfrak{r}+1)}\left(\frac{\mathrm{M}_{1}}{1-\mathrm{N}_{1}-\mathrm{N}_{2} \frac{\mathrm{T}^{\sigma}}{\sigma \Gamma(\delta)}}+\frac{\mathrm{M}_{2} \frac{\mathrm{T}^{\sigma}}{\sigma \Gamma(\delta)}}{1-\mathrm{N}_{1}-\mathrm{N}_{2} \frac{\mathrm{T}^{\sigma}}{\sigma \Gamma(\delta)}}\right)\right.\right. \\
& \left.+A_{\mathrm{h}}+m A_{I_{i}}\right]\left\|x-\chi^{\star}\right\|_{\mathcal{M}} .
\end{aligned}
$$

Which yields

$$
\left\|x-x^{*}\right\|_{\mathcal{M}} \leq \frac{\epsilon_{\mathfrak{r}}\left(\psi_{\mathfrak{r}}(t)+\phi_{\mathfrak{r}}\right)\left(\varrho_{\psi_{\mathfrak{r}}}(1+\mathcal{K})+\mathcal{K}\right)}{1-\left[\frac{(1+m) T^{\mathrm{r}}}{\Gamma(\mathfrak{r}+1)}\left(\frac{\mathrm{M}_{1}}{1-\mathrm{N}_{1}-\mathrm{N}_{2} \frac{T^{\sigma}}{\sigma \Gamma(\delta)}}+\frac{\mathrm{M}_{2} \frac{\mathrm{T}^{\sigma}}{\sigma \Gamma(\delta)}}{1-\mathrm{N}_{1}-\mathrm{N}_{2} \frac{T^{\sigma}}{\sigma \Gamma(\delta)}}\right)+A_{\mathrm{h}}+m A_{I_{i}}\right]} .
$$

Similarly, for $t \in \mathcal{J}_{0}$, we have

$$
\left\|x-x^{*}\right\|_{\mathcal{M}} \leq \frac{\epsilon_{\mathfrak{r}}\left(\psi_{\mathfrak{r}}(t)+\phi_{\mathfrak{r}}\right) \varrho_{\psi_{\mathfrak{r}}}}{1-\left[\frac{\mathrm{T}^{\mathfrak{r}}}{\Gamma(\mathfrak{r}+1)}\left(\frac{\mathrm{M}_{1}}{1-\mathrm{N}_{1}-\mathrm{N}_{2} \frac{T^{\sigma}}{\sigma \Gamma(\delta)}}+\frac{\mathrm{M}_{2} \frac{\mathrm{T}^{\sigma}}{\sigma \Gamma(\delta)}}{1-\mathrm{N}_{1}-\mathrm{N}_{2} \frac{T^{\sigma}}{\sigma \Gamma(\delta)}}\right)+A_{\mathrm{h}}\right]} .
$$

Combining (4.8) and (4.9), for $t \in \mathcal{J}$, we have

$$
\begin{aligned}
\left\|x-x^{\star}\right\|_{\mathcal{M}} \leq & \epsilon_{\mathfrak{r}}\left(\psi_{\mathfrak{r}}(t)+\phi_{\mathfrak{r}}\right)\left[\frac{\varrho_{\psi_{\mathfrak{r}}}(1+\mathcal{K})+\mathcal{K}}{1-\left[\frac{(1+m) \mathrm{T}^{\mathfrak{r}}}{\Gamma(\mathfrak{r}+1)}\left(\frac{\mathrm{M}_{1}}{1-\mathrm{N}_{1}-\mathrm{N}_{2} \frac{T^{\sigma}}{\sigma \Gamma(\delta)}}+\frac{\mathrm{M}_{2} \frac{T^{\sigma}}{\sigma \Gamma(\delta)}}{1-\mathrm{N}_{1}-\mathrm{N}_{2} \frac{T^{\sigma}}{\sigma \Gamma(\delta)}}\right)+A_{\mathrm{h}}+m A_{I_{i}}\right]}\right. \\
& +\frac{\varrho_{\psi_{\mathfrak{r}}}}{1-\left[\frac{\mathrm{T}^{\mathfrak{r}}}{\Gamma(\mathfrak{r}+1)}\left(\frac{\mathrm{M}_{1}}{\left.\left.1-\mathrm{N}_{1}-\mathrm{N}_{2} \frac{\mathrm{I}^{\sigma}}{\sigma \Gamma(\delta)}+\frac{\mathrm{M}_{2} \frac{\mathrm{T}^{\sigma}}{\sigma \Gamma(\delta)}}{1-\mathrm{N}_{1}-\mathrm{N}_{2} \frac{T^{\sigma}}{\sigma \Gamma(\delta)}}\right)+A_{\mathrm{h}}\right]}\right]\right.} .
\end{aligned}
$$

Thus

$$
\left\|x-\chi^{\star}\right\|_{\mathcal{M}} \leq \mathbf{C}_{2} \epsilon_{\mathfrak{r}}(\psi(t)+\phi)
$$


where

$$
\begin{aligned}
& \mathbf{C}_{2}= {\left[\frac{\varrho_{\psi}(1+\mathcal{K})+\mathcal{K}}{1-\left[\frac{(1+m) T^{\mathfrak{r}}}{\Gamma(\mathfrak{r}+1)}\left(\frac{\mathrm{M}_{1}}{1-\mathrm{N}_{1}-\mathrm{N}_{2} \frac{T^{\sigma}}{\sigma \Gamma(\delta)}}+\frac{\mathrm{M}_{2} \frac{\mathrm{T}^{\sigma}}{\sigma \Gamma(\delta)}}{1-\mathrm{N}_{1}-\mathrm{N}_{2} \frac{T^{\sigma}}{\sigma \Gamma(\delta)}}\right)+A_{\mathrm{h}}+m A_{I_{i}}\right]}\right.} \\
&+\frac{\varrho_{\psi}}{1-\left[\frac{\mathrm{r}}{\Gamma(\mathfrak{r}+1)}\left(\frac{\mathrm{M}_{1}}{1-\mathrm{N}_{1}-\mathrm{N}_{2} \frac{\mathrm{T}^{\sigma}}{\sigma \Gamma(\delta)}}+\frac{\mathrm{M}_{2} \frac{\mathrm{T}^{\sigma}}{\sigma \Gamma(\delta)}}{1-\mathrm{N}_{1}-\mathrm{N}_{2} \frac{T^{\sigma}}{\sigma \Gamma(\delta)}}\right)+A_{\mathrm{h}}\right]} .
\end{aligned}
$$

Therefore, problem (1.1) is HU-Rassias stable. Similarly, we can show that it is generalized HU-Rassias stable.

Next, we study the stability results of the proposed system (1.2). The following definitions are adopted from [7].

Let $\epsilon_{\mathfrak{r}}, \epsilon_{\mathfrak{s}}>0, \mathcal{F}, \mathcal{G}, \mathcal{F}^{\prime}, \mathcal{G}^{\prime}$ be continuous functions and $\psi_{\mathfrak{r}}, \psi_{\mathfrak{s}}: \mathfrak{J} \rightarrow \mathbb{R}^{+}$are nondecreasing functions. Consider the following inequalities:

$$
\begin{aligned}
& \left\{\begin{array}{l}
\left|{ }^{c} D^{\mathfrak{r}} x(t)-\mathcal{F}\left(t, y(t),{ }^{c} D^{\mathfrak{r}} x(t)\right)-\int_{0}^{t} \frac{(t-\xi)}{\Gamma(\delta)} \mathcal{G}\left(\xi, y(\xi),{ }^{c} D^{\mathfrak{r}} x(\xi)\right) d \xi\right| \leq \epsilon_{\mathfrak{r}}, \forall t \in \mathcal{J}, \\
\left|{ }^{c} D^{\mathfrak{s}} y(t)-\mathcal{F}^{\prime}\left(t, x(t),{ }^{c} D^{\mathfrak{s}} y(t)\right)-\int_{0}^{t} \frac{(t-\xi)}{\Gamma(\delta)} \mathcal{G}^{\sigma-1}\left(\xi, x(\xi),{ }^{c} D^{\mathfrak{s}} y(\xi)\right) d \xi\right| \leq \epsilon_{\mathfrak{s}}, \forall t \in \mathcal{J}, \\
\left|\Delta x\left(t_{i}\right)-I_{i}\left(x\left(t_{i}\right)\right)\right| \leq \epsilon_{\mathfrak{r}}, \quad i=1,2, \ldots, m, \\
\left|\Delta y\left(t_{j}\right)-I_{j}\left(y\left(t_{j}\right)\right)\right| \leq \epsilon_{\mathfrak{s}}, \quad j=1,2, \ldots, n,
\end{array}\right. \\
& \left\{\begin{array}{l}
\left|{ }^{c} D^{\mathfrak{r}} x(t)-\mathcal{F}\left(t, y(t),{ }^{c} D^{\mathfrak{r}} x(t)\right)-\int_{0}^{t} \frac{(t-\xi))^{\sigma-1}}{\Gamma(\delta)} \mathcal{G}\left(\xi, y(\xi),{ }^{c} D^{\mathfrak{r}} x(\xi)\right) d \xi\right| \leq \psi_{\mathfrak{r}}(t), \forall t \in \mathcal{J}, \\
\left|{ }^{c} D^{\mathfrak{s}} y(t)-\mathcal{F}^{\prime}\left(t, x(t),{ }^{c} D^{\mathfrak{s}} y(t)\right)-\int_{0}^{t} \frac{(t-\xi)^{\sigma-1}}{\Gamma(\delta)} \mathcal{G}^{\prime}\left(\xi, x(\xi),{ }^{c} D^{\mathfrak{s}} y(\xi)\right) d \xi\right| \leq \psi_{\mathfrak{s}}(t), \forall t \in \mathcal{J}, \\
\left|\Delta x\left(t_{i}\right)-I_{i}\left(x\left(t_{i}\right)\right)\right| \leq \phi_{\mathfrak{r}}, \quad i=1,2, \ldots, m, \\
\left|\Delta y\left(t_{j}\right)-I_{j}\left(y\left(t_{j}\right)\right)\right| \leq \phi_{\mathfrak{s}}, \quad j=1,2, \ldots, n
\end{array}\right.
\end{aligned}
$$

and

$$
\left\{\begin{array}{l}
\left|{ }^{c} D^{\mathfrak{r}} x(t)-\mathcal{F}\left(t, y(t),{ }^{c} D^{\mathfrak{r}} x(t)\right)-\int_{0}^{t} \frac{(t-\xi)}{\Gamma(\delta)} \mathcal{G}\left(\xi, y(\xi),{ }^{c} D^{\mathfrak{r}} x(\xi)\right) d \xi\right| \leq \epsilon_{\mathfrak{r}} \psi_{\mathfrak{r}}(t), \forall t \in \mathcal{J}, \\
\left|{ }^{c} D^{\mathfrak{s}} y(t)-\mathcal{F}^{\prime}\left(t, x(t),{ }^{c} D^{\mathfrak{s}} y(t)\right)-\int_{0}^{t} \frac{(t-\xi)}{\Gamma(\delta)} \mathcal{G}^{\prime}\left(\xi, x(\xi),{ }^{c} D^{\mathfrak{s}} y(\xi)\right) d \xi\right| \leq \epsilon_{\mathfrak{s}} \psi_{\mathfrak{s}}(t), \forall t \in \mathcal{J}, \\
\left|\Delta x\left(t_{i}\right)-I_{i}\left(x\left(t_{i}\right)\right)\right| \leq \epsilon_{\mathfrak{r}} \phi_{\mathfrak{r}}, \quad i=1,2, \ldots, m, \\
\left|\Delta y\left(t_{j}\right)-I_{j}\left(y\left(t_{j}\right)\right)\right| \leq \epsilon_{\mathfrak{s}} \phi_{\mathfrak{s}}, \quad j=1,2, \ldots, n .
\end{array}\right.
$$

Definition 4.12. The problem (1.2) is said to be HU stable, if there is $\mathcal{C}_{\mathfrak{r}, \mathfrak{s}}=\left(\mathcal{C}_{\mathfrak{r}}, \mathcal{C}_{\mathfrak{s}}\right)>0$ for some $\epsilon=\left(\epsilon_{\mathfrak{r}}, \epsilon_{\mathfrak{s}}\right)$ and for each solution $(x, y) \in X \times y$ of (4.10), there is a solution $\left(x^{*}, y^{\star}\right) \in X \times y$ of problem (1.2) with

$$
\left|(x, y)(t)-\left(x^{\star}, y^{\star}\right)(t)\right| \leq \mathcal{C}_{\mathfrak{r}, \mathfrak{s}} \epsilon, \forall t \in \mathcal{J} .
$$

Definition 4.13. The problem (1.2) is said to be generalized HU stable, if there is $\Theta \in C(\mathcal{J}, \mathbb{R})$ with $\Theta(0)=0$, so that for each solution $(x, y) \in \mathcal{X} \times y$ of (4.10), there is a solution $\left(x^{\star}, y^{\star}\right) \in X \times Y$ of problem (1.2) with

$$
\left|(x, y)(t)-\left(x^{\star}, y^{\star}\right)(t)\right| \leq \Theta(\epsilon), \forall t \in \mathcal{J} .
$$


Definition 4.14. The problem (1.2) is said to be HU-Rassias stable with respect to $\psi_{\mathfrak{r}, \mathfrak{s}}=\left(\psi_{\mathfrak{r}}, \psi_{\mathfrak{s}}\right) \in C^{1}(\mathcal{J}, \mathbb{R})$, if there is a constant $\mathcal{C}_{\psi_{\mathfrak{r}}, \psi_{\mathfrak{s}}}=\left(\mathcal{C}_{\psi_{\mathfrak{r}}}, \mathcal{C}_{\psi_{\mathfrak{s}}}\right)$ so that for some $\epsilon=\left(\epsilon_{\mathfrak{r}}, \epsilon_{\mathfrak{s}}\right)>0$ and for each solution $(x, y) \in \mathcal{X} \times y$ of (4.11), there is a solution $\left(x^{\star}, y^{\star}\right) \in \mathcal{X} \times y$ of problem (1.2) with

$$
\left|(x, y)(t)-\left(x^{\star}, y^{\star}\right)(t)\right| \leq \mathcal{C}_{\psi_{\mathrm{r}}, \psi_{\mathrm{s}}} \epsilon, \forall t \in \mathcal{J} .
$$

Definition 4.15. The problem (1.2) is said to be generalized HU-Rassias stable with respect to $\psi_{\mathfrak{r}, \mathfrak{s}}=\left(\psi_{\mathfrak{r}}, \psi_{\mathfrak{s}}\right)$, if there is a constant $\mathcal{C}_{\psi_{\mathrm{r}}, \psi_{\mathrm{s}}}=\left(\mathcal{C}_{\psi_{\mathrm{r}}}, \mathcal{C}_{\psi_{\mathrm{s}}}\right)>0$, so that for each solution $(x, y) \in \mathcal{X} \times \mathcal{Y}$ of (4.12), there is a solution $\left(x^{\star}, y^{\star}\right) \in X \times y$ of problem (1.2) with

$$
\left|(x, y)(t)-\left(x^{\star}, y^{\star}\right)(t)\right| \leq \mathcal{C}_{\psi_{\mathfrak{r}}, \psi_{\mathfrak{s}}} \psi_{\mathfrak{r}, \mathfrak{s}}, \forall t \in \mathcal{J} .
$$

Remark 4.16. Definition 4.12 implies Definition 4.13 and Definition 4.14 implies Definition 4.15.

Remark 4.17. We say that $(x, y) \in X \times y$ is a solution of (4.10) if there are functions $\mu_{\mathcal{F}, \mathcal{G}}, \Lambda_{\mathcal{F}^{\prime}, \mathcal{G}^{\prime}} \in X \times y$ which depend upon $(x, y)$ respectively, so that

i) $\left|\mu_{\mathcal{F}, \mathcal{G}}(t)\right| \leq \epsilon_{\mathfrak{r}},\left|\Lambda_{\mathcal{F}^{\prime}, \mathcal{G}^{\prime}}(t)\right| \leq \epsilon_{\mathfrak{s}}, \forall t \in \mathcal{J}$;

ii)

$$
{ }^{c} D^{\mathfrak{r}} x(t)=\mathcal{F}\left(t, y(t),{ }^{c} D^{\mathfrak{r}} x(t)\right)+\int_{0}^{t} \frac{(t-\xi)}{\Gamma(\delta)} \mathcal{G}\left(\xi, y(\xi),{ }^{c} D^{\mathfrak{r}} x(\xi)\right) d \xi+\mu_{\mathcal{F}, \mathcal{G}}(t), t \in \mathcal{J}_{i} ;
$$

and

$$
{ }^{c} D^{\mathfrak{s}} y(t)=\mathcal{F}\left(t, x(t),{ }^{c} D^{\mathfrak{s}} y(t)\right)+\int_{0}^{t} \frac{(t-\xi)^{\sigma-1}}{\Gamma(\delta)} \mathcal{G}\left(\xi, x(\xi),{ }^{c} D^{\mathfrak{s}} y(\xi)\right) d \xi+\Lambda_{\mathcal{F}^{\prime}, \mathcal{G}^{\prime}}(t), t \in \mathcal{J}_{j} ;
$$

iii) $\Delta x\left(t_{i}\right)=I_{i}\left(x\left(t_{i}\right)\right)+\mu_{i}, t \in \partial_{i}, i=1,2, \ldots, m$ and $\Delta y\left(t_{j}\right)=I_{j}\left(y\left(t_{j}\right)\right)+\Lambda_{j}, t \in \mathcal{J}_{j}, j=1,2, \ldots, n$.

Theorem 4.18. Let $(x, y) \in X \times y$ be the solution of the inequality (4.10), then we have

$$
\left\{\begin{array}{l}
\left|x(t)-x^{\star}(t)\right| \leq\left[\frac{(1+m) t^{\mathfrak{r}}}{\Gamma(\mathfrak{r}+1)}+m\right] \epsilon_{\mathfrak{r}}, \\
\left|y(t)-y^{\star}(t)\right| \leq\left[\frac{(1+n) t^{\mathfrak{s}}}{\Gamma(\mathfrak{s}+1)}+n\right] \epsilon_{\mathfrak{s}} .
\end{array}\right.
$$

Proof. Let $(x, y)$ be the solution of the inequality (4.10), then by Remark 4.17. $(x, y)$ will also be the solution of

$$
\left\{\begin{array}{l}
{ }^{c} D^{\mathfrak{r}} x(t)=\mathcal{F}\left(t, y(t),{ }^{c} D^{\mathfrak{r}} x(t)\right)+\int_{0}^{t} \frac{(t-\xi)^{\sigma-1}}{\Gamma(\delta)} \mathcal{G}\left(\xi, y(\xi),{ }^{c} D^{\mathfrak{r}} x(\xi)\right) d \xi+\mu_{\mathcal{F}, \mathcal{G}}, \forall t \in \mathcal{J}, t \neq t_{i} \text { for } i=1,2, \ldots, m, \\
{ }^{c} D^{\mathfrak{s}} y(t)=\mathcal{F}^{\prime}\left(t, x(t),{ }^{c} D^{\mathfrak{s}} y(t)\right)+\int_{0}^{t} \frac{(t-\xi)^{\sigma-1}}{\Gamma(\delta)} \mathcal{G}^{\prime}\left(\xi, x(\xi),{ }^{c} D^{\mathfrak{s}} y(\xi)\right) d \xi+\Lambda_{\mathcal{F}^{\prime}, \mathcal{G}^{\prime}}, \forall t \in \mathcal{J}, t \neq t_{j} \text { for } j=1,2, \ldots, n, \\
x(0)=\mathrm{h}(x), y(0)=\mathrm{g}(y), \\
\Delta x\left(t_{i}\right)=I_{i}\left(x\left(t_{i}\right)\right)+\mu_{i}, \quad i=1,2, \ldots, m, \\
\Delta y\left(t_{j}\right)=I_{j}\left(y\left(t_{j}\right)\right)+\Lambda_{j}, \quad j=1,2, \ldots, n .
\end{array}\right.
$$


i.e,

$$
x(t)=\left\{\begin{array}{l}
\frac{1}{\Gamma(\mathfrak{r})} \int_{0}^{t}(t-\xi)^{\mathfrak{r}-1} \alpha(\xi) d \xi+\frac{1}{\Gamma(\mathfrak{r})} \int_{0}^{t}(t-\xi)^{\mathfrak{r}-1} \mu(\xi) d \xi+\mathrm{h}(x), t \in \mathrm{J}_{0}, \\
\frac{1}{\Gamma(\mathfrak{r})} \int_{t_{i}}^{t}(t-\xi)^{\mathfrak{r}-1} \alpha(\xi) d \xi+\frac{1}{\Gamma(r)} \int_{t_{i}}^{t}(t-\xi)^{\mathfrak{r}-1} \mu(\xi) d \xi+\sum_{i=1}^{m} \frac{1}{\Gamma(\mathfrak{r})} \int_{t_{i-1}}^{t_{i}}\left(t_{i}-\xi\right)^{\mathfrak{r}-1} \alpha(\xi) d \xi \\
+\sum_{i=1}^{m} \frac{1}{\Gamma(\mathfrak{r})} \int_{t_{i-1}}^{t_{i}}\left(t_{i}-\xi\right)^{\mathfrak{r}-1} \mu(\xi) d \xi+\sum_{i=1}^{m} I_{i}\left(x\left(t_{i}\right)\right)+\sum_{i=1}^{m} \mu_{i}+\mathrm{h}(x), t \in J_{i}, i=1,2, \ldots, m
\end{array}\right.
$$

and

$$
y(t)=\left\{\begin{array}{l}
\frac{1}{\Gamma(\mathfrak{s})} \int_{0}^{t}(t-\xi)^{\mathfrak{s}-1} \beta(\xi) d \xi+\frac{1}{\Gamma(\mathfrak{s})} \int_{0}^{t}(t-\xi)^{\mathfrak{s}-1} \Lambda(\xi) d \xi+\mathrm{g}(y), t \in \mathcal{J}_{0}, \\
\frac{1}{\Gamma(\mathfrak{s})} \int_{t_{j}}^{t}(t-\xi)^{\mathfrak{s}-1} \beta(\xi) d \xi+\frac{1}{\Gamma(\mathfrak{s})} \int_{t_{j}}^{t}(t-\xi)^{\mathfrak{s}-1} \Lambda(\xi) d \xi+\sum_{j=1}^{n} \frac{1}{\Gamma(\mathfrak{s})} \int_{t_{j-1}}^{t_{j}}\left(t_{j}-\xi\right)^{\mathfrak{s}-1} \beta(\xi) d \xi \\
+\sum_{i=1}^{n} \frac{1}{\Gamma(\mathfrak{s})} \int_{t_{j-1}}^{t_{j}}\left(t_{j}-\xi\right)^{\mathfrak{s}-1} \Lambda(\xi) d \xi+\sum_{j=1}^{n} I_{j}\left(y\left(t_{j}\right)\right)+\sum_{j=1}^{n} \Lambda_{j}+\mathrm{g}(y), t \in \mathcal{J}_{j}, j=1,2, \ldots, n,
\end{array}\right.
$$

where

$$
\alpha(t)=\mathcal{F}\left(t, y(t),{ }^{c} D^{\mathfrak{r}} x(t)\right)+\int_{0}^{t} \frac{(t-\xi)^{\sigma-1}}{\Gamma(\delta)} \mathcal{G}\left(\xi, y(\xi),{ }^{c} D^{\mathfrak{r}} x(\xi)\right) d \xi
$$

and

$$
\beta(t)=\mathcal{F}^{\prime}\left(t, x(t),{ }^{c} D^{\mathfrak{s}} y(t)\right)+\int_{0}^{t} \frac{(t-\xi)}{\Gamma(\delta)} \mathcal{G}^{\prime}\left(\xi, x(\xi),{ }^{c} D^{\mathfrak{s}} y(\xi)\right) d \xi .
$$

From (4.18), we have

$$
x(t)=\left\{\begin{array}{l}
\frac{1}{\Gamma(\mathfrak{r})} \int_{0}^{t}(t-\xi)^{\mathfrak{r}-1} \alpha(\xi) d \xi+\frac{1}{\Gamma(\mathfrak{r})} \int_{0}^{t}(t-\xi)^{\mathfrak{r}-1} \mu(\xi) d \xi+\mathrm{h}(x), t \in \mathcal{J}_{0}, \\
\frac{1}{\Gamma(\mathfrak{r})} \int_{t_{i}}^{t}(t-\xi)^{\mathfrak{r}-1} \alpha(\xi) d \xi+\frac{1}{\Gamma(\mathfrak{r})} \int_{t_{i}}^{t}(t-\xi)^{\mathfrak{r}-1} \mu(\xi) d \xi+\sum_{i=1}^{m} \frac{1}{\Gamma(\mathfrak{r})} \int_{t_{i-1}}^{t_{i}}\left(t_{i}-\xi\right)^{\mathfrak{r}-1} \alpha(\xi) d \xi \\
+\sum_{i=1}^{m} \frac{1}{\Gamma(\mathfrak{r})} \int_{t_{i-1}}^{t_{i}}\left(t_{i}-\xi\right)^{\mathfrak{r}-1} \mu(\xi) d \xi+\sum_{i=1}^{m} I_{i}\left(x\left(t_{i}\right)\right)+\sum_{i=1}^{m} \mu_{i}+\mathrm{h}(x), t \in J_{i}, i=1,2, \ldots, m .
\end{array}\right.
$$

Thus (4.20) becomes

$$
\left|x(t)-x^{\star}(t)\right| \leq \frac{1}{\Gamma(\mathfrak{r})} \int_{t_{i}}^{t}(t-\xi)^{\mathfrak{r}-1}|\mu(\xi)| d \xi+\sum_{i=1}^{m} \frac{1}{\Gamma(\mathfrak{r})} \int_{t_{i-1}}^{t_{i}}\left(t_{i}-\xi\right)^{\mathfrak{r}-1}|\mu(\xi)| d \xi+\sum_{i=1}^{m}\left|\mu_{i}\right|,
$$

where

$$
x^{\star}(t)=\frac{1}{\Gamma(\mathfrak{r})} \int_{t_{i}}^{t}(t-\xi)^{\mathfrak{r}-1} \alpha(\xi) d \xi+\sum_{i=1}^{m}\left[\frac{1}{\Gamma(\mathfrak{r})} \int_{t_{i-1}}^{t_{i}}(t-\xi)^{\mathfrak{r}-1} \alpha(\xi) d \xi+I_{i}\left(x^{\star}\left(t_{i}\right)\right)\right]+\mathrm{h}\left(x^{\star}\right), i=1,2, \ldots, m .
$$


Using (i) of Remark 4.17, we obtain

$$
\left|x(t)-\chi^{\star}(t)\right| \leq\left[\frac{(1+m) t^{\mathfrak{r}}}{\Gamma(\mathfrak{r}+1)}+m\right] \epsilon_{\mathfrak{r}} .
$$

Repeating the similar procedure for (4.19) together with (i) from Remark 4.17, we have

$$
\left|y(t)-y^{\star}(t)\right| \leq\left[\frac{(1+n) t^{\mathfrak{s}}}{\Gamma(\mathfrak{s}+1)}+n\right] \epsilon_{\mathfrak{s}},
$$

where

$$
y^{\star}(t)=\frac{1}{\Gamma(\mathfrak{s})} \int_{t_{j}}^{t}(t-\xi)^{\mathfrak{s}-1} \beta(\xi) d \xi+\sum_{j=1}^{n}\left[\frac{1}{\Gamma(\mathfrak{s})} \int_{t_{j-1}}^{t_{j}}\left(t_{j}-\xi\right)^{\mathfrak{s}-1} \beta(\xi) d \xi+I_{j}\left(y^{\star}\left(t_{j}\right)\right)\right]+\mathrm{g}\left(y^{\star}\right), j=1,2, \ldots, n .
$$

Theorem 4.19. If the hypothesis $\left[H_{1}\right]-\left[H_{4}\right],\left[H_{8}\right]$ and $\left[H_{9}\right]$ satisfies with

$$
\Delta=1-\mathrm{Q}_{\mathfrak{r}} \mathrm{Q}_{\mathfrak{s}}>0,
$$

then system (1.2) is stable in the sense of $\mathrm{HU}$.

Proof. Suppose $(x, y) \in X \times y$ be the solution of the inequality (4.12) and $\left(x^{\star}, y^{\star}\right) \in X \times y$ is the solution of the given system

$$
\left\{\begin{array}{l}
{ }^{c} D^{\mathfrak{r}} x^{\star}(t)=\mathcal{F}\left(t, y^{\star}(t),{ }^{c} D^{\mathfrak{r}} x^{\star}(t)\right)+\int_{0}^{t} \frac{(t-\xi)^{\sigma-1}}{\Gamma(\delta)} \mathcal{G}\left(\xi, y^{\star}(\xi),{ }^{c} D^{\mathfrak{r}} x^{\star}(\xi)\right) d \xi, \forall t \in \mathcal{J}, t \neq t_{i} \text { for } i=1,2, \ldots, m, \\
{ }^{c} D^{\mathfrak{s}} y^{\star}(t)=\mathcal{F}^{\prime}\left(t, x^{\star}(t),{ }^{c} D^{\mathfrak{s}} y^{\star}(t)\right)+\int_{0}^{t} \frac{(t-\xi)^{\sigma-1}}{\Gamma(\delta)} \mathcal{G}^{\prime}\left(\xi, x^{\star}(\xi),{ }^{c} D^{\mathfrak{s}} y^{\star}(\xi)\right) d \xi, \forall t \in \mathcal{J}, t \neq t_{j} \text { for } j=1,2, \ldots, n, \\
x^{\star}(0)=\mathrm{h}\left(x^{\star}\right), y^{\star}(0)=\mathrm{g}\left(y^{\star}\right), \\
\Delta x^{\star}\left(t_{i}\right)=I_{i}\left(x^{\star}\left(t_{i}\right)\right), \quad i=1,2, \ldots, m, \\
\Delta y^{\star}\left(t_{j}\right)=I_{j}\left(y^{\star}\left(t_{j}\right)\right), \quad j=1,2, \ldots, n .
\end{array}\right.
$$

Then in view of Theorem 3.5, the solution of (4.22) is

$$
\chi^{\star}(t)=\frac{1}{\Gamma(\mathfrak{r})} \int_{t_{i}}^{t}(t-\xi)^{\mathfrak{r}-1} \alpha(\xi) d \xi+\sum_{i=1}^{m}\left[\frac{1}{\Gamma(\mathfrak{r})} \int_{t_{i-1}}^{t_{i}}(t-\xi)^{\mathfrak{r}-1} \alpha(\xi) d \xi+I_{i}\left(\chi^{*}\left(t_{i}\right)\right)\right]+\mathrm{h}\left(\chi^{\star}\right), i=1,2, \ldots, m
$$

and

$$
y^{\star}(t)=\frac{1}{\Gamma(\mathfrak{s})} \int_{t_{j}}^{t}(t-\xi)^{\mathfrak{s}-1} \beta(\xi) d \xi+\sum_{j=1}^{n}\left[\frac{1}{\Gamma(\mathfrak{s})} \int_{t_{j-1}}^{t_{j}}\left(t_{j}-\xi\right)^{\mathfrak{s}-1} \beta(\xi) d \xi+I_{j}\left(y^{\star}\left(t_{j}\right)\right)\right]+\mathrm{g}\left(y^{\star}\right), j=1,2, \ldots, n,
$$

where

$$
\alpha(t)=\mathcal{F}\left(t, y^{\star}(t),{ }^{c} D^{\mathfrak{r}} x^{\star}(t)\right)+\int_{0}^{t} \frac{(t-\xi)^{\sigma-1}}{\Gamma(\delta)} \mathcal{G}\left(\xi, y^{\star}(\xi),{ }^{c} D^{\mathfrak{r}} x^{\star}(\xi)\right) d \xi
$$

and

$$
\beta(t)=\mathcal{F}^{\prime}\left(t, x^{\star}(t),{ }^{c} D^{\mathfrak{s}} y^{\star}(t)\right)+\int_{0}^{t} \frac{(t-\xi)^{\sigma-1}}{\Gamma(\delta)} \mathcal{G}^{\prime}\left(\xi, x^{\star}(\xi),{ }^{c} D^{\mathfrak{s}} y^{\star}(\xi)\right) d \xi
$$


Consider

$$
\begin{aligned}
\left|x(t)-x^{\star}(t)\right| \leq & |x(t)-q(t)|+\left|q(t)-x^{\star}(t)\right| \\
\leq & {\left[\frac{(1+m) t^{\mathfrak{r}}}{\Gamma(\mathfrak{r}+1)}+m\right] \epsilon_{\mathfrak{r}}+\frac{1}{\Gamma(\mathfrak{r})} \int_{t_{i}}^{t}(t-\xi)^{\mathfrak{r}-1}\left|\tilde{x}(\xi)-\tilde{x}^{\star}(\xi)\right| d \xi+\sum_{i=1}^{m} \frac{1}{\Gamma(\mathfrak{r})} \int_{t_{i-1}}^{t_{i}}\left(t_{i}-\xi\right)^{\mathfrak{r}-1}\left|\tilde{x}(\xi)-\tilde{x}^{\star}(\xi)\right| d \xi } \\
& +\sum_{i=1}^{m}\left|I_{i}\left(x\left(t_{i}\right)\right)-I_{i}\left(x^{\star}\left(t_{i}\right)\right)\right|+\left|\mathrm{h}(x)-\mathrm{h}\left(x^{\star}\right)\right|
\end{aligned}
$$

where

$$
\tilde{x}(t)=\mathcal{F}(t, y(t), \tilde{x}(t))+\int_{0}^{t} \frac{(t-\xi)^{\sigma-1}}{\Gamma(\delta)} \mathcal{G}(\xi, y(\xi), \tilde{x}(\xi)) d \xi
$$

and

$$
\tilde{x}^{\star}(t)=\mathcal{F}\left(t, y^{\star}(t), \tilde{x}^{\star}(t)\right)+\int_{0}^{t} \frac{(t-\xi)^{\sigma-1}}{\Gamma(\delta)} \mathcal{G}\left(\xi, y^{\star}(\xi), \tilde{x}^{\star}(\xi)\right) d \xi .
$$

Using $\left[H_{1}\right]$, we have

$$
\begin{aligned}
\left|\tilde{x}(t)-\tilde{x}^{*}(t)\right|= & \mid \mathcal{F}(t, y(t), \tilde{x}(t))+\int_{0}^{t} \frac{(t-\xi)^{\sigma-1}}{\Gamma(\delta)} \mathcal{G}(\xi, y(\xi), \tilde{x}(\xi)) d \xi \\
& -\mathcal{F}\left(t, y^{\star}(t), \tilde{x}^{\star}(t)\right)-\int_{0}^{t} \frac{(t-\xi)^{\sigma-1}}{\Gamma(\delta)} \mathcal{G}\left(\xi, y^{\star}(\xi), \tilde{x}^{\star}(\xi)\right) d \xi \mid \\
\leq & \left|\mathcal{F}(t, y(t), \tilde{x}(t))-\mathcal{F}\left(t, y^{\star}(t), \tilde{x}^{*}(t)\right)\right|+\int_{0}^{t} \frac{(t-\xi)^{\sigma-1}}{\Gamma(\delta)}\left|\mathcal{G}(\xi, y(\xi), \tilde{x}(\xi))-\mathcal{G}\left(\xi, y^{*}(\xi), \tilde{x}^{*}(\xi)\right)\right| d \xi \\
\leq & \mathrm{M}_{1}\left|y(t)-y^{\star}(t)\right|+\mathrm{N}_{1}\left|\tilde{x}(t)-\tilde{x}^{\star}(t)\right|+\frac{t^{\sigma}}{\sigma \Gamma(\delta)}\left(\mathrm{M}_{2}\left|y(t)-y^{\star}(t)\right|+\mathrm{N}_{2}\left|\tilde{x}(t)-\tilde{x}^{\star}(t)\right|\right) .
\end{aligned}
$$

Thus

$$
\left|\tilde{x}(t)-\tilde{x}^{\star}(t)\right| \leq\left(\frac{\mathrm{M}_{1}}{1-\mathrm{N}_{1}-\mathrm{N}_{2} \frac{t^{\sigma}}{\sigma \Gamma(\delta)}}+\frac{\mathrm{M}_{2} \frac{t^{\sigma}}{\sigma \Gamma(\delta)}}{1-\mathrm{N}_{1}-\mathrm{N}_{2} \frac{t^{\sigma}}{\sigma \Gamma(\delta)}}\right)\left|y(t)-y^{\star}(t)\right| .
$$

Using hypothesis $\left(\mathrm{H}_{3}\right),\left(\mathrm{H}_{4}\right)$ and (4.24) in (4.23), we have

$$
\begin{aligned}
\left|x(t)-x^{\star}(t)\right| \leq & {\left[\frac{(1+m) t^{\mathfrak{r}}}{\Gamma(\mathfrak{r}+1)}+m\right] \epsilon_{\mathfrak{r}}+\left[\left(\frac{(1+m) t^{\mathfrak{r}}}{\Gamma(\mathfrak{r}+1)}\right)\left(\frac{\mathrm{M}_{1}}{1-\mathrm{N}_{1}-\mathrm{N}_{2} \frac{t^{\sigma}}{\sigma \Gamma(\delta)}}+\frac{\mathrm{M}_{2} \frac{t^{\sigma}}{\sigma \Gamma(\delta)}}{1-\mathrm{N}_{1}-\mathrm{N}_{2} \frac{t^{\sigma}}{\sigma \Gamma(\delta)}}\right)\right]\left|y(t)-y^{\star}(t)\right| } \\
& +\left(m A_{I_{i}}+A_{\mathrm{h}}\right)\left|x(t)-x^{\star}(t)\right| .
\end{aligned}
$$

By taking norm and simplification, we get

$$
\begin{aligned}
\left\|x-x^{\star}\right\| x \leq & {\left[\frac{(1+m) T^{\mathfrak{v}}}{\Gamma(\mathfrak{r}+1)}+m\right] \epsilon_{\mathfrak{r}}+\left[\left(\frac{(1+m) \mathrm{T}^{\mathfrak{r}}}{\Gamma(\mathfrak{r}+1)}\right)\left(\frac{\mathrm{M}_{1}}{1-\mathrm{N}_{1}-\mathrm{N}_{2} \frac{\mathrm{T}^{\sigma}}{\sigma(\delta)}}+\frac{\mathrm{M}_{2} \frac{\mathrm{T}^{\sigma}}{\sigma \Gamma(\delta)}}{1-\mathrm{N}_{1}-\mathrm{N}_{2} \frac{\mathrm{T}^{\sigma}}{\sigma \Gamma(\delta)}}\right)\right]\left\|y-y^{\star}\right\| x } \\
& +\left(m A_{I_{i}}+A_{\mathrm{h}}\right)\left\|x-x^{\star}\right\| x .
\end{aligned}
$$

For simplicity, we consider

$$
S_{\mathfrak{r}}=\frac{\left[\frac{(1+m) \mathrm{T}^{\mathfrak{r}}}{\Gamma(\mathfrak{r}+1)}+m\right]}{1-\left(m A_{I_{i}}+A_{\mathrm{h}}\right)}
$$




$$
\mathrm{Q}_{\mathfrak{r}}=\frac{\left[\left(\frac{(1+m) \mathrm{T}^{\mathfrak{r}}}{\Gamma(\mathrm{r}+1)}\right)\left(\frac{\mathrm{M}_{1}}{1-\mathrm{N}_{1}-\mathrm{N}_{2} \frac{\mathrm{T}^{\sigma}}{\sigma \Gamma(\delta)}}+\frac{\mathrm{M}_{2} \frac{\mathrm{T}^{\sigma}}{\Gamma \Gamma(\delta)}}{1-\mathrm{N}_{1}-\mathrm{N}_{2} \frac{T^{\sigma}}{\sigma \Gamma(\delta)}}\right)\right]}{1-\left(m A_{I_{i}}+A_{\mathrm{h}}\right)},
$$

(4.25) implies

$$
\left\|x-x^{\star}\right\| x \leq \mathrm{S}_{\mathfrak{r}} \epsilon_{\mathfrak{r}}+\mathrm{Q}_{\mathfrak{r}}\left\|y-y^{\star}\right\| x
$$

and similarly

$$
\left\|y-y^{\star}\right\| y \leq \mathrm{S}_{\mathfrak{s}} \epsilon_{\mathfrak{s}}+\mathrm{Q}_{\mathfrak{s}}\left\|x-x^{\star}\right\| y,
$$

where

$$
\begin{aligned}
& \mathrm{S}_{\mathfrak{s}}=\frac{\left[\frac{(1+n) \mathrm{T}^{\mathfrak{s}}}{\Gamma(\mathfrak{s}+1)}+n\right]}{1-\left(n A_{I_{j}}+A_{\mathrm{g}}\right)}, \\
& \mathrm{Q}_{\mathfrak{s}}=\frac{\left[\left(\frac{(1+n) T^{\mathfrak{S}}}{\Gamma(\mathfrak{s}+1)}\right)\left(\frac{\mathrm{M}_{1}^{\prime}}{1-\mathrm{N}_{1}^{\prime}-\mathrm{N}_{2}^{\prime} \frac{T^{\sigma}}{\sigma \Gamma(\delta)}}+\frac{\mathrm{M}_{2}^{\prime} \frac{\mathrm{T}^{\sigma}}{\sigma \Gamma(\delta)}}{1-\mathrm{N}_{1}^{\prime}-\mathrm{N}_{2}^{\prime} \frac{T^{\sigma}}{\sigma \Gamma(\delta)}}\right)\right]}{1-\left(n A_{I_{j}}+A_{\mathrm{g}}\right)}, \\
& \left\|x-x^{\star}\right\|_{x}-\mathrm{Q}_{\mathfrak{r}}\left\|y-y^{*}\right\|_{x} \leq \mathrm{S}_{\mathfrak{r}} \epsilon_{\mathfrak{r}}, \\
& \left\|y-y^{\star}\right\| y-Q_{\mathfrak{s}}\left\|x-x^{\star}\right\|_{y} \leq \mathrm{S}_{\mathfrak{s}} \epsilon_{\mathfrak{s}} \text {, } \\
& {\left[\begin{array}{cc}
1 & -Q_{\mathfrak{r}} \\
-Q_{\mathfrak{s}} & 1
\end{array}\right]\left[\begin{array}{c}
\left\|x-x^{\star}\right\|_{x \times y} \\
\left\|y-y^{\star}\right\|_{x \times y}
\end{array}\right] \leq\left[\begin{array}{c}
\mathcal{S}_{\mathfrak{r}} \epsilon_{\mathfrak{r}} \\
\mathcal{S}_{\mathfrak{s}} \epsilon_{\mathfrak{s}}
\end{array}\right] .}
\end{aligned}
$$

Solving the above inequality, we have

$$
\left[\begin{array}{l}
\left\|x-x^{\star}\right\|_{x \times y} \\
\left\|y-y^{\star}\right\|_{x \times y}
\end{array}\right] \leq\left[\begin{array}{cc}
\frac{1}{\Delta} & \frac{Q_{\mathfrak{r}}}{\Delta} \\
\frac{\mathrm{Q}_{\mathfrak{s}}}{\Delta} & \frac{1}{\Delta}
\end{array}\right]\left[\begin{array}{c}
\mathcal{S}_{\mathfrak{r}} \epsilon_{\mathfrak{r}} \\
\mathcal{S}_{\mathfrak{s}} \epsilon_{\mathfrak{s}}
\end{array}\right],
$$

where

$$
\Delta=1-Q_{\mathfrak{r}} Q_{\mathfrak{s}}>0 .
$$

Further simplification gives

$$
\begin{aligned}
& \left\|x-x^{\star}\right\|_{x \times y} \leq \frac{\mathrm{S}_{\mathfrak{r}} \epsilon_{\mathfrak{r}}}{\Delta}+\frac{\mathrm{Q}_{\mathfrak{r}} \mathrm{S}_{\mathfrak{s}} \epsilon_{\mathfrak{s}}}{\Delta}, \\
& \left\|y-y^{\star}\right\|_{x \times y} \leq \frac{\mathrm{S}_{\mathfrak{s}} \epsilon_{\mathfrak{s}}}{\Delta}+\frac{\mathrm{Q}_{\mathfrak{s}} \mathrm{S}_{\mathfrak{r}} \epsilon_{\mathfrak{r}}}{\Delta} .
\end{aligned}
$$

From which we have

$$
\left\|x-x^{*}\right\| x_{x} y+\left\|y-y^{*}\right\|_{x \times y} \leq \frac{\mathrm{S}_{\mathfrak{r}} \epsilon_{\mathfrak{r}}}{\Delta}+\frac{\mathrm{S}_{\mathfrak{s}} \epsilon_{\mathfrak{s}}}{\Delta}+\frac{\mathrm{Q}_{\mathfrak{r}} \mathrm{S}_{\mathfrak{s}} \epsilon_{\mathfrak{s}}}{\Delta}+\frac{\mathrm{Q}_{\mathfrak{s}} \mathrm{S}_{\mathfrak{r}} \epsilon_{\mathfrak{r}}}{\Delta} .
$$

Let $\max \left\{\epsilon_{\mathfrak{r}}, \epsilon_{\mathfrak{s}}\right\}=\epsilon$, then from (4.28) we get

$$
\left\|(x, y)-\left(x^{\star}, y^{\star}\right)\right\|_{x \times y} \leq C_{\mathfrak{r}, \mathfrak{s}} \epsilon,
$$

where

$$
C_{3}=\left[\frac{\mathrm{S}_{\mathfrak{r}}}{\Delta}+\frac{\mathrm{S}_{\mathfrak{s}}}{\Delta}+\frac{\mathrm{Q}_{\mathfrak{r}} \mathrm{S}_{\mathfrak{s}}}{\Delta}+\frac{\mathrm{Q}_{\mathfrak{s}} \mathrm{S}_{\mathfrak{r}}}{\Delta}\right] .
$$

This completes the proof.

Remark 4.20. We set $\Theta(\epsilon)=C_{3} \epsilon, \Theta(0)=0$ in (4.28). By Definition 4.13 the proposed system (1.2) is generalized HU stable. 
In order to obtain the connections between the HU-Rassias stability concepts we introduce the following hypothesis.

- $\left[H_{10}\right]$ Let $\Omega_{\mathfrak{r}}, \Omega_{\mathfrak{s}} \in C\left(\mathcal{J}, \mathbb{R}^{+}\right)$be an increasing functions. Then there exist $\Lambda_{\Omega_{\mathfrak{r}}}, \Lambda_{\Omega_{\mathfrak{s}}}>0$ such that for each $t \in \mathcal{J}$ the integral inequalities:

$$
I^{\mathfrak{r}} \Omega_{\mathfrak{r}}(t) \leq \Lambda_{\Omega_{\mathfrak{r}}} \Omega_{\mathfrak{r}}(t) \text { and } I^{\mathfrak{r}-1} \Omega_{\mathfrak{r}}(t) \leq \Lambda_{\Omega_{\mathfrak{r}}} \Omega_{\mathfrak{r}}(t)
$$

and

$$
I^{\mathfrak{s}} \Omega_{\mathfrak{s}(t)} \leq \Lambda_{\Omega_{\mathfrak{s}}} \Omega_{\mathfrak{s}}(t) \text { and } I^{\mathfrak{s}-1} \Omega_{\mathfrak{s}}(t) \leq \Lambda_{\Omega_{\mathfrak{s}}} \Omega_{\mathfrak{s}}(t)
$$

holds.

Under the hypothesis $\left[H_{1}\right]-\left[H_{10}\right],(4.21)$ with Theorem 4.18 and Theorem 4.19 system (1.2) will be HURassias and generalized HU-Rassias stable.

\section{Illustrative example}

\section{Example 5.1.}

$$
\left\{\begin{array}{l}
{ }^{c} D^{\frac{1}{2}} x(t)=\frac{1+|x(t)|+\left.\cos \right|^{c} D^{\frac{1}{2}} x(t) \mid}{104 e^{t+5}\left(1+|x(t)|+\left|{ }^{c} D^{\frac{1}{2}} x(t)\right|\right)}+\int_{0}^{1} \frac{(t-\xi)^{\frac{3}{2}}}{\Gamma\left(\frac{5}{2}\right)} \frac{1+|x(\xi)|+\sin \left|{ }^{c} D^{\frac{1}{2}} x(\xi)\right|}{104 e^{t+5}\left(1+|x(\xi)|+\left|{ }^{c} D^{\frac{1}{2}} x(\xi)\right|\right)} d \xi, t \in[0,1], \\
x(0)=\mathrm{h}(x)=\frac{\cos |x(t)|}{18+e^{t}} \\
I_{1}\left(x\left(\frac{1}{2}\right)\right)=\frac{\left|x\left(\frac{1}{2}\right)\right|}{40+\left|x\left(\frac{1}{2}\right)\right|},
\end{array}\right.
$$

where $\mathfrak{r}=\frac{1}{2}, \partial_{0}=\left[0, \frac{1}{2}\right], J_{1}=\left(\frac{1}{2}, 1\right], \sigma=\delta=\frac{5}{2}$. Set

$$
\begin{aligned}
& \mathcal{F}(t, x, y)=\frac{1+|x(t)|+\cos \left|{ }^{c} D^{\frac{1}{2}} x(t)\right|}{104 e^{t+5}\left(1+|x(t)|+\left|{ }^{c} D^{\frac{1}{2}} x(t)\right|\right)}, \forall t \in[0,1], \\
& \mathcal{G}(t, x, y)=\frac{1+|x(t)|+\left.\sin \right|^{c} D^{\frac{1}{2}} x(t) \mid}{104 e^{t+5}\left(1+|x(t)|+\left|{ }^{c} D^{\frac{1}{2}} x(t)\right|\right)}, \forall t \in[0,1] .
\end{aligned}
$$

Obviously, $\mathcal{F}$ and $\mathcal{G}$ are jointly continuous functions.

Now, for each $x, \bar{x} \in \mathcal{M}$ and $y, \bar{y} \in \mathbb{R}$ and $t \in[0,1]$, we have

$$
|\mathcal{F}(t, x, y)-\mathcal{F}(t, \bar{x}, \bar{y})| \leq \frac{1}{104 e^{5}}(|x-\bar{x}|+|y-\bar{y}|)
$$

and

$$
|\mathcal{G}(t, x, y)-\mathcal{G}(t, \bar{x}, \bar{y})| \leq \frac{1}{104 e^{5}}(|x-\bar{x}|+|y-\bar{y}|) .
$$

Which satisfies $\left[A_{1}\right]$ with $\mathrm{M}_{1}=\mathrm{M}_{2}=\mathrm{N}_{1}=\mathrm{N}_{2}=\frac{1}{104 e^{5}}$. Set

$$
I_{1}\left(x\left(\frac{1}{3}\right)\right)=\frac{\left|x\left(\frac{1}{3}\right)\right|}{40+\left|x\left(\frac{1}{3}\right)\right|}, \forall x \in \mathcal{M} .
$$

Then for $x, \bar{x} \in \mathcal{M}$, we have

$$
\left|I_{1}\left(x\left(\frac{1}{3}\right)\right)-I_{1}\left(\bar{x}\left(\frac{1}{3}\right)\right)\right|=\left|\frac{\left|x\left(\frac{1}{3}\right)\right|}{40+\left|x\left(\frac{1}{3}\right)\right|}-\frac{\left|\bar{x}\left(\frac{1}{3}\right)\right|}{40+\left|\bar{x}\left(\frac{1}{3}\right)\right|}\right| \leq \frac{1}{35}|x-\bar{x}| .
$$

Hence, with $A_{I_{1}}=\frac{1}{35},\left[A_{2}\right]$ satisfies. Set

$$
\mathrm{h}(x)=\frac{\cos |x(t)|}{18+e^{t}}
$$


Then for $x, \bar{x} \in \mathbb{R}$, we have

$$
|\mathrm{h}(x)-\mathrm{h}(\bar{x})|=\left|\frac{\cos |x(t)|}{18+e^{t}}-\frac{\cos |\bar{x}(t)|}{18+e^{t}}\right| \leq \frac{1}{19}|x-\bar{x}| .
$$

Hence, $\left[A_{3}\right]$ satisfies with $A_{\mathrm{h}}=\frac{1}{19}$. Also

$$
\left[\left(\frac{(1+m) \mathrm{T}^{\mathfrak{r}}}{\Gamma(\mathfrak{r}+1)}\right)\left(\frac{\mathrm{M}_{1}}{1-\mathrm{N}_{1}-\mathrm{N}_{2} \frac{\mathrm{T}^{\sigma}}{\sigma \Gamma(\delta)}}+\frac{\mathrm{M}_{2} \frac{\mathrm{T}^{\sigma}}{\sigma \Gamma(\delta)}}{1-\mathrm{N}_{1}-\mathrm{N}_{2} \frac{\mathrm{T}^{\sigma}}{\sigma \Gamma(\delta)}}\right)+m A_{I_{1}}+A_{\mathrm{h}}\right] \approx 0.08138<1
$$

satisfies with $m=1, \mathrm{~T}=1, \sigma=\delta=\frac{5}{2}, \mathfrak{r}=\frac{1}{2}, \mathrm{M}_{1}=\mathrm{N}_{1}=\mathrm{M}_{2}=\mathrm{N}_{2}=\frac{1}{104 e^{5}}, A_{I_{1}}=\frac{1}{35}, A_{\mathrm{h}}=\frac{1}{19}$. Therefore, by Theorem 3.4, the problem (5.1) has a unique solution.

Now to confirm the existence of at least one solution, we need to check $\left[A_{4}\right]$ and $\left[A_{6}\right]$.

For each $t \in[0,1]$, we have

$$
\left|\mathcal{F}\left(t, x(t),{ }^{c} D^{\frac{1}{2}} x(t)\right)\right| \leq \frac{1}{104 e^{t+5}}\left(1+|u(t)+\cos t|^{c} D^{\frac{1}{2}} x(t)||\right) .
$$

Hence, $\left[A_{4}\right]$ satisfies with

$$
a_{1}(t)=\frac{1}{38 e^{t+5}}, b_{1}(t)=c_{1}(t)=\frac{1}{104 e^{t+5}}
$$

Also

$$
\left|\mathcal{G}\left(t, x(t),{ }^{c} D^{\frac{1}{2}} x(t)\right)\right| \leq \frac{1}{104 e^{t+5}}\left(1+|u(t)|+\left.\sin \right|^{c} D^{\frac{1}{2}} x(t) \mid\right) .
$$

Hence, $\left[A_{4}\right]$ satisfies with

$$
a_{2}(t)=\frac{1}{38 e^{t+5}}, b_{2}(t)=c_{2}(t)=\frac{1}{104 e^{t+5}}
$$

And for each $x \in \mathcal{M}$, we have

$$
\left|I_{i} x(t)\right| \leq \frac{1}{35}|x(t)|+1
$$

Hence, $\left[A_{6}\right]$ also satisfies with $\mathcal{K}=\frac{1}{35}$ and $\mathcal{L}=1$.

Therefore, by Theorem 3.3, the problem (5.1) has at least one solution on the given interval.

Let for any $t \in[0,1]$, we have $\psi(t)=|t|$ and $\phi=1$. Then

$$
I^{\frac{1}{2}} \chi(t)=\frac{1}{\Gamma\left(\frac{1}{2}\right)} \int_{0}^{t}(t-\xi)^{\frac{1}{2}-1}|\xi| d \xi \leq \frac{2 t}{\sqrt{\pi}} .
$$

Thus, $\left[A_{7}\right]$ satisfies with $\varrho_{\psi_{\frac{1}{2}}}=\frac{2}{\sqrt{\pi}}$. Therefore, by Theorem 4.10, the problem (5.1) is HU-Rassias stable and consequently, it is generalized HU-Rassias stable.

\section{Example 5.2.}

$$
\left\{\begin{array}{l}
{ }^{c} D^{\frac{1}{2}} x(t)=\frac{1+|y(t)|+\cos \left|{ }^{c} D^{\frac{1}{2}} x(t)\right|}{104 e^{t+5}\left(1+|y(t)|+\left|{ }^{c} D^{\frac{1}{2}} x(t)\right|\right)}+\int_{0}^{1} \frac{(t-\xi)^{\frac{3}{2}}}{\Gamma\left(\frac{5}{2}\right)} \frac{1+|y(\xi)|+\sin \left|{ }^{c} D^{\frac{1}{2}} x(\xi)\right|}{104 e^{t+5}\left(1+|y(\xi)|+\left|{ }^{c} D^{\frac{1}{2}} x(\xi)\right|\right)} d \xi, t \in[0,1], t \neq \frac{1}{3}, \\
{ }^{c} D^{\frac{1}{2}} y(t)=\frac{2+|x(t)|+\left.\cos \right|^{c} D^{\frac{1}{2}} y(t) \mid}{70 e^{t+2}\left(1+|x(t)|+\left|{ }^{c} D^{\frac{1}{2}} y(t)\right|\right)}+\int_{0}^{1} \frac{(t-\xi)^{\frac{3}{2}}}{\Gamma\left(\frac{5}{2}\right)} \frac{|x(\xi)|+\cos \left|{ }^{c} D^{\frac{1}{2}} y(\xi)\right|}{70 e^{t+2}\left(1+|x(\xi)|+\left|{ }^{c} D^{\frac{1}{2}} y(\xi)\right|\right)} d \xi, t \in[0,1], t \neq \frac{1}{4}, \\
x(0)=\mathrm{h}(x)=\frac{\cos |x(t)|}{18+e^{t}}, y(0)=\mathrm{g}(y)=\frac{\sin |y(t)|}{18+e^{t}}, \\
I_{1}\left(x\left(\frac{1}{3}\right)\right)=\frac{\left|x\left(\frac{1}{3}\right)\right|}{40+\left|x\left(\frac{1}{3}\right)\right|}, I_{1}\left(y\left(\frac{1}{4}\right)\right)=\frac{1}{50+\left|y\left(\frac{1}{4}\right)\right|},
\end{array}\right.
$$

$t_{i}=\frac{1}{3}$ for $i=1,2,3, \ldots, 60$, and $t_{j}=\frac{1}{4}$ for $j=1,2,3, \ldots, 100$.

For any $x, \bar{x}, y, \bar{y} \in \mathbb{R}, t \in[0,1]$, we obtain

$$
|\mathcal{F}(t, x, y)-\mathcal{F}(t, \bar{x}, \bar{y})| \leq \frac{1}{104 e^{5}}(|x-\bar{x}|+|y-\bar{y}|)
$$


and

$$
|\mathcal{G}(t, x, y)-\mathcal{G}(t, \bar{x}, \bar{y})| \leq \frac{1}{104 e^{5}}(|x-\bar{x}|+|y-\bar{y}|) .
$$

Similarly, For any $x, \bar{x}, y, \bar{y} \in \mathbb{R}, t \in[0,1]$, we obtain

$$
\left|\mathcal{F}^{\prime}(t, x, y)-\mathcal{F}^{\prime}(t, \bar{x}, \bar{y})\right| \leq \frac{1}{70 e^{2}}(|x-\bar{x}|+|y-\bar{y}|)
$$

and

$$
\left|\mathcal{G}^{\prime}(t, x, y)-\mathcal{G}^{\prime}(t, \bar{x}, \bar{y})\right| \leq \frac{1}{70 e^{2}}(|x-\bar{x}|+|y-\bar{y}|) .
$$

Which satisfies $\left[H_{1}\right]$ with $M_{1}=M_{2}=N_{1}=N_{2}=\frac{1}{104 e^{5}}, M_{1}^{\prime}=M_{2}^{\prime}=N_{1}^{\prime}=N_{2}^{\prime}=\frac{1}{70 e^{2}}$. Set

$$
I_{i}\left(x\left(\frac{1}{3}\right)\right)=\frac{\left|x\left(\frac{1}{3}\right)\right|}{40+\left|x\left(\frac{1}{3}\right)\right|}, \forall x \in X .
$$

Then for $x, \bar{x} \in \mathcal{X}$, we have

$$
\left|I_{i}\left(x\left(\frac{1}{3}\right)\right)-I_{i}\left(\bar{x}\left(\frac{1}{3}\right)\right)\right|=\left|\frac{\left|x\left(\frac{1}{3}\right)\right|}{40+\left|x\left(\frac{1}{3}\right)\right|}-\frac{\left|\bar{x}\left(\frac{1}{3}\right)\right|}{40+\left|\bar{x}\left(\frac{1}{3}\right)\right|}\right| \leq \frac{1}{35}|x-\bar{x}| .
$$

Hence, with $A_{I_{i}}=\frac{1}{35}$.

Similarly,

$$
I_{j}\left(y\left(\frac{1}{4}\right)\right)=\frac{\left|y\left(\frac{1}{4}\right)\right|}{50+|y|}, \forall y \in y .
$$

Then for $y, \bar{y} \in y$, we have

$$
\left|I_{j}\left(y\left(\frac{1}{4}\right)\right)-I_{j}\left(\bar{y}\left(\frac{1}{4}\right)\right)\right|=\left|\frac{\left|y\left(\frac{1}{4}\right)\right|}{50+|y|}-\frac{\left|\bar{y}\left(\frac{1}{4}\right)\right|}{50+\mid \bar{y}}\right| \leq \frac{1}{50}|y-\bar{y}| .
$$

Hence, with $A_{I_{j}}=\frac{1}{50}$, the $\left[H_{3}\right]$ satisfies. Set

$$
\mathrm{h}(x)=\frac{\cos |x(t)|}{18+e^{t}} .
$$

Then for $x, \bar{x} \in \mathbb{R}$, we have

$$
|\mathrm{h}(x)-\mathrm{h}(\bar{x})|=\left|\frac{\cos |x(t)|}{18+e^{t}}-\frac{\cos |\bar{x}(t)|}{18+e^{t}}\right| \leq \frac{1}{19}|x-\bar{x}| .
$$

Hence, $\left[H_{4}\right]$ satisfies with $A_{\mathrm{h}}=\frac{1}{19}$.

Similarly, Set

$$
\mathrm{g}(y)=\frac{\sin |x(t)|}{100+e^{2 t}}
$$

Then for $y, \bar{y} \in \mathbb{R}$, we have

$$
|\mathrm{g}(y)-\mathrm{g}(\bar{y})|=\left|\frac{\sin |y(t)|}{100+e^{2 t}}-\frac{\sin |\bar{y}(t)|}{100+e^{2 t}}\right| \leq \frac{1}{101}|y-\bar{y}| .
$$

Hence, $\left[H_{4}\right]$ satisfies with $A_{\mathrm{g}}=\frac{1}{101}$.

Also

$$
\Delta_{1}=\left[\left(\frac{(1+m) \mathrm{T}^{\mathfrak{r}}}{\Gamma(\mathfrak{r}+1)}\right)\left(\frac{\mathrm{M}_{1}}{1-\mathrm{N}_{1}-\mathrm{N}_{2} \frac{\mathrm{T}^{\sigma}}{\sigma \Gamma(\delta)}}+\frac{\mathrm{M}_{2} \frac{\mathrm{T}^{\sigma}}{\sigma \Gamma(\delta)}}{1-\mathrm{N}_{1}-\mathrm{N}_{2} \frac{T^{\sigma}}{\sigma \Gamma(\delta)}}\right)+m A_{I_{i}}+A_{\mathrm{h}}\right] \approx 0.08138<1
$$

satisfies with $m=1, \mathrm{~T}=1, \sigma=\delta=\frac{5}{2}, \mathfrak{r}=\frac{1}{2}, \mathrm{M}_{1}=\mathrm{N}_{1}=\mathrm{M}_{2}=\mathrm{N}_{2}=\frac{1}{104 \mathrm{e}^{5}}, A_{I_{i}}=\frac{1}{35}, A_{\mathrm{h}}=\frac{1}{19}$ and

$$
\Delta_{2}=\left[\left(\frac{(1+n) \mathrm{T}^{\mathfrak{s}}}{\Gamma(\mathfrak{s}+1)}\right)\left(\frac{\mathrm{M}_{1}^{\prime}}{1-\mathrm{N}_{1}^{\prime}-\mathrm{N}_{2}^{\prime} \frac{\mathrm{T}^{\sigma}}{\sigma \Gamma(\delta)}}+\frac{\mathrm{M}_{2}^{\prime} \frac{\mathrm{T}^{\sigma}}{\sigma \Gamma(\delta)}}{1-\mathrm{N}_{1}^{\prime}-\mathrm{N}_{2}^{\prime} \frac{\mathrm{T}^{\sigma}}{\sigma \Gamma(\delta)}}\right)+n A_{I_{j}}+A_{\mathrm{g}}\right] \approx 0.03559<1
$$


satisfies with $n=1, \mathrm{~T}=1, \sigma=\delta=\frac{5}{2}, \mathfrak{s}=\frac{1}{2}, \mathrm{M}_{1}^{\prime}=\mathrm{N}_{1}^{\prime}=\mathrm{M}_{2}^{\prime}=\mathrm{N}_{2}^{\prime}=\frac{1}{70 e^{2}}, A_{I_{j}}=\frac{1}{50}, A_{\mathrm{g}}=\frac{1}{101}$. Hence $\Delta=\max \left(\Delta_{1}, \Delta_{2}\right)<1$ satisfies.

Therefore, by Theorem 3.8, the problem (5.2) has a unique solution. It is easy to check that

$$
\Delta=1-Q_{\mathfrak{r}} Q_{\mathfrak{s}} \approx 1.00000>0
$$

and (4.21) is verified. We conclude that problem (5.2) is HU stable, generalized HU stable, HU-Rassias stable and generalized HU-Rassias stable.

\section{Conclusion}

We have presented some existence and uniqueness results for an impulsive initial value problem of coupled fractional integrodifferential systems involving the Caputo type fractional derivative. The proof of the existence results is based on the nonlinear alternative of Schaefer's and Krasnoselskii's fixed point theorem, while the uniqueness of the solution is proved by applying the Banach contraction principle. We have also given the notion of Hyers-Ulam stability for our problem and have given sufficient conditions for EUS and Hyers-Ulam stability. This work provides a base to the study of EUS and different sorts of stabilities for the fractional integrodifferential equations with impulsive initial condition.

Acknowledgments: The third author would like to thank Prince Sultan University for funding this work through research group Nonlinear Analysis Methods in Applied Mathematics (NAMAM) group number RGDES-2017-01-17. The research of the fourth author was supported by the Natural Science Foundation of Jiangxi Province (grant:20192BAB201011) and by the National Natural Science Foundation of China (grant:11861053).

\section{References}

[1] Dalir M., Bashour M., Applications of fractional calculus, Appl. Math. Sci., 2010, 4, 1021-1032

[2] Khan H., Khan A., Abdeljawad T., Alkhazzan A., Existence results in Banach space for a nonlinear impulsive system, Adv. Difference Equ., 2019, 2019:18

[3] Khan A., Gómez-Aguilar J. F., Khan T. S., Khan H., Stability analysis and numerical solutions of fractional order HIV/AIDS model, Chaos Solitons Fractals, 2019, 122, 119-128

[4] Khan H., Abdeljawad T., Aslam M., Khan R. A., Khan A., Existence of positive solution and Hyers-Ulam stability for a nonlinear singular-delay-fractional differential equation, Adv. Difference Equ., 2019, 2019:104

[5] Khan H., Gómez-Aguilar J. F., Khan A., Khan T. S., Stability analysis for fractional order advection reaction diffusion system, Phys. A, 2019, 521, 737-751

[6] Hilfer R., Applications of Fractional Calculus in Physics, World Scientific, River Edge, NJ, USA, 2000

[7] Meral F., Royston T., Magin R., Fractional calculus in viscoelasticity: an experimental study, Commun. Nonlinear Sci. Numer. Simul., 2010, 15, 939-945

[8] Benchohra M., Graef J. R., Hamani S., Existence results for boundary value problems with nonlinear fractional differential equations, Appl. Anal., 2008, 87, 851-863

[9] Abdeljawad T., Fractional operators with exponential kernels and a Lyapunov type inequality, Adv. Difference Equ., 2017, 2017:313

[10] Babakhani A., Abdeljawad T., A Caputo fractional order boundary value problem with integral boundary conditions, J. Comput. Anal. Appl., 2013, 15(4), 753-763

[11] Abdeljawad T., Jarad F., Baleanu D., On the existence and the uniqueness theorem for fractional differential equations with bounded delay within Caputo derivatives, Science in China, Mathematics, 2008, 51, 1775-1786

[12] Abdeljawad T., Baleanu D., Jarad F., Existence and uniqueness theorem for a class of delay differential equations with left and right Caputo fractional derivatives, J. Math. Phys., 2008, 49(8), 083507-083507-11

[13] Abdeljawad T., Al-Mdallal Q. M., Discrete Mittag-Leffer kernel type fractional difference initial value problems and Gronwalls inequality, J. Comput. Appl. Math., 2018, 339, 218-230

[14] Kilbas A. A., Marichev O. I., Samko S. G., Fractional integrals and derivatives (theory and applications), Switzerland: Gordonand Breach, 1993

[15] Miller K. S., Ross B., An Introduction to the Fractional Calculus and Fractional Differential Equations, New York: Wiley, 1993 
[16] Rehman M., Khan R., A note on boundary value problems for a coupled system of fractional differential equations, Comput. Math Appl., 2011, 61, 2630-2637

[17] Zada A., Ali S., Stability analysis of multi-point boundary value problem for sequential fractional differential equations with non-instantaneous impulses, Int. J. Nonlinear Sci. Numer. Simul., 2018, 19, 763-774

[18] Kilbas A. A., Srivastava H. M., Trujillo J. J., Theory and Applications of Fractional Differential Equations, vol. 204 of NorthHolland Mathematics Studies, Elsevier Science B. V., Amsterdam, The Netherlands, 2006

[19] Podlubny I., Fractional Differential Equations, Academic Press, New York, 1999

[20] Ahmad B., Nieto J. J., Existence results for nonlinear boundary value problems of fractional integro-differential equations with integral boundary conditions, Boun. Value Prob., 2009, 2009:708576

[21] Chalishajar D. N., Karthikeyan K., Boundary value problems for impulsive fractional evolution integrodifferential equations with Gronwall's inequality in Banach spaces, J. Dis. Nonl. Compl., 2014, 3, 33-48

[22] Chalishajar D. N., Karthikeyan K., Existence and uniqueness results for boundary value problems of higher order fractional integro-differential equations involving Gronwall's inequality in Banach spaces, Acta Math. Sci. Ser., 2013, 33, 758-772

[23] Khan A., Shah K., Li Y., Khan T. S., Ulam type stability for a coupled system of boundary value problems of nonlinear fractional differential, J. Funct. Spaces, 2017, Article ID 3046013

[24] Muslim M., Kumar A., Agarwal R. P., Exact controllability of fractional integro-differential systems of order $\alpha \in(1,2]$ with deviated argument, Analele Universitatii Oradea, XXIV, 2017, 59, 185-194

[25] Shah R., Zada A., A fixed point approach to the stability of a nonlinear volterra integrodifferential equations with delay, Hacettepe J. Math. Stat., 2018, 47, 615-623

[26] Ahmad B., Nieto J. J., Existence results for a coupled system of nonlinear fractional differential equations with three-point boundary conditions, Comp. Math. Appl., 2009, 58, 1838-1843

[27] Laskin N., Fractional market dynamics, Phys. A, 2000, 287, 482-492

[28] Lin W., Global existence theory and chaos control of fractional differential equations, J. Math. Anal. Appl., 2007, 332, 709726

[29] Ravichandran C., Logeswari K., Jarad F., New results on existence in the framework of Atangana-Baleanu derivative for fractional integro-differential equations, Chaos Solitons Fractals, 2019, 125, 194-200

[30] Gambo Y. Y., Ameen R., Jarad F., Abdeljawad T., Existence and uniqueness of solutions to fractional differential equations in the frame of generalized Caputo fractional derivatives, Adv. Difference Equ., 2018, 2018:134

[31] Chalishajar D., Kumar A., Existence, uniqueness and Ulam's stability of solutions for a coupled system of fractional differential equations with integral boundary conditions, Mathematics, 2018, 6, 96

[32] Alzabut J., Almost periodic solutions for impulsive delay Nicholsons blowflies population model, J. Comput. Appl. Math., 2010, 234, 233-239

[33] Georgieva A., Kostadinov S., Stamov G. T., Alzabut J. O., On $L_{p}(k)$ equivalence of impulsive differential equations and its applications to partial impulsive differential equations, Adv. Difference Equ., 2012, 2012:144

[34] Lakshmikantham V., Leela S., Vasundhara J., Theory of fractional dynamic systems, Cambridge, UK: Cambridge Academic Publishers, 2009

[35] Lakshmikanthan V., Bainov D. D., Simeonov P.S., Theory of impulsive differential equations, Singapore: World Scientific, 1989

[36] Lupulescu V., Zada A., Linear impulsive dynamic systems on time scales, Electron. J. Qual. Theory Differ. Equ., 2010, 11, 1-30

[37] Tang S., Zada A., Faisal S., El-Sheikh M. M. A., Li T., Stability of higher-order nonlinear impulsive differential equations, J. Nonlinear Sci. Appl., 2016, 9, 4713-4721

[38] Wang J., Zada A., Ali W., Ulam's-type stability of first-order impulsive differential equations with variable delay in quasiBanach spaces, Int. J. Nonlin. Sci. Num., 2018, 19, 553-560

[39] Zada A., Faisal S., Li Y., On the Hyers-Ulam stability of first order impulsive delay differential equations, J. Funct. Spaces., 2016, Article ID 8164978

[40] Zada A., Riaz U., Khan F. U., Hyers-Ulam stability of impulsive integral equation, Boll. Unione Mat. Ital., 2019, 12(3), 453-467

[41] Zada A., Mashal A., Stability analysis of $n^{\text {th }}$ order nonlinear impulsive differential equations in quasi-Banach space, Numer. Funct. Anal. Optim., 2019, DOI:10.1080/01630563.2019.1628049

[42] Bainov D., Dimitrova M., Dishliev A., Oscillation of the bounded solutions of impulsive differential difference equations of second order, Appl. Math. Comput., 2000, 114, 61-68

[43] Chernousko F., Akulenko L., Sokolov B., Control of Oscilations, Moskow: Nauka, 1980

[44] Chua L. O., Yang L., Cellular neural networks: applications, IEEE Trans. Circ. syst., 1998, 35, 1273-1290

[45] Stamov G. Tr., Alzabut J. O., Almost periodic solutions of impulsive integrodifferential neural networks, Math. Model. Analysis, 2010, 15, 505-516

[46] Popov E., The Dynamics of Automatic Control Systems, Moskow: Goste-hizdat, 1964

[47] Andronov A., Witt A., Haykin S., Oscilation Theory, Moskow: Nauka, 1981

[48] Zavalishchin S., Sesekin A., Impulsive Processes: Models and Applications, Moskow: Nauka, 1991

[49] Babitskii V., Krupenin V., Vibration in Strongly Nonlinear Systems, Moskow: Nauka, 1985

[50] Stamov G. Tr., Alzabut J. O., Atanasov P., Stamov A. G., Almost periodic solutions for an impulsive delay model of price fluctuations in commodity markets, Nonlinear Anal. RWA, 2011, 12, 3170-3176 
[51] Wang J., Zhang Y., Ulam-Hyers-Mittag-Leffler stability of fractional-order delay differential equations, Optimization, 2014, 63(8), 1181-1190

[52] Li T., Zada A., Connections between Hyers-Ulam stability and uniform exponential stability of discrete evolution families of bounded linear operators over Banach spaces, Adv. Difference Equ., 2016, 2016:153

[53] Zada A., Wang P., Lassoued D., Li T. X., Connections between Hyers-Ulam stability and uniform exponential stability of 2periodic linear nonautonomous systems, Adv. Difference Equ., 2017, 2017:192

[54] Ali S., Abdeljawad T., Shah K., Jarad F., Arif M., Computation of iterative solutions along with stability analysis to a coupled system of fractional order differential equations, Adv. Difference Equ., 2019, 2019:215

[55] Ali A., Shah K., Jarad F., Gupta V., Abdeljawad T., Existence and stability analysis to a coupled system of implicit type impulsive boundary value problems of fractional-order differential equations, Adv. Difference Equ., 2019, 2019:101

[56] Jarad F., Abdeljawad T., Hammouch Z., On a class of ordinary differential equations in the frame of Atangana-Baleanu fractional derivative, Chaos Solitons Fractals, 2018, 117, 16-20

[57] Asma, Ali A., Shah K., Jarad F., Ulam-Hyers stability analysis to a class of nonlinear implicit impulsive fractional differential equations with three point boundary conditions, Adv. Difference Equ., 2019, 2019:7

[58] Ameena R., Jaradb F., Abdeljawad T., Ulam stability for delay fractional differential equations with a generalized Caputo derivative, Filomat, 2018, 32, 5265-5274

[59] Hyers D. H., On the stability of the linear functional equation, Proc. Natl. Acad. Sci., 1941, 27, 222-224

[60] Ulam S. M., Problems in Modern Mathematics, Courier Corporation, 2004.

[61] Zada A., Shah S. O., Shah R., Hyers-Ulam stability of non-autonomous systems in terms of boundedness of Cauchy problem, Appl. Math. Comput., 2015, 271, 512-518

[62] Zada A., Shah S. O., Hyers-Ulam stability of first-order non-linear delay differential equations with fractional integrable impulses, Hacettepe J. Math. Stat., 2018, 47, 1196-1205

[63] Wang J., Zhou Y., Wei W., Study in fractional differential equations by means of topological degree methods, Num. Func. Anal. Opti., 2012, 33, 216-238

[64] Tian Y., Ba Z., Impulsive boundary value problem for differential equations with fractional order, Differ. Equ. Dyn. Syst., 2013, $21,253-260$

[65] Zhang X., Zhu C., Wu Z., Solvability for a coupled system of fractional differential equations with impulsis at resonance, Boun. Value Prob., 2013, 2013:80

[66] Shah K., Khalil H., Khan R. A., Investigation of positive solution to a coupled system of impulsive boundary value problems for nonlinear fractional order differential equations, Elsevier Science B. V., Amsterdam, The Netherlands, 2015, 77, 240-246

[67] Benchohra M., Lazreg J. E., On the stability of nonlinear implicit fractional differential equations, Le Matematiche, 2015, 70, 49-61

[68] Zada A., Ali S., Li Y., Ulam-type stability for a class of implicit fractional differential equations with non-instantaneous integral impulses and boundary condition, Adv. Difference Equ., 2017, 2017:317

[69] Guo D., Lakshmikantham V., Nonlinear Problems in Abstract Cone, Academic Press, Orlando, 1988

[70] Yurko V. A., Boundary value problems with discontinuity conditions in an interior point of the interval, J. Diff. Equa., 2000, 36, 1266-1269 